\title{
Experimental Measurement of Dielectric Properties of Powdery Materials using a Coaxial Transmission Line
}

\author{
Robert Tempke \\ rstempke@mix.wvu.edu
}

Follow this and additional works at: https://researchrepository.wvu.edu/etd

Part of the Mechanical Engineering Commons, and the Other Materials Science and Engineering Commons

\section{Recommended Citation}

Tempke, Robert, "Experimental Measurement of Dielectric Properties of Powdery Materials using a Coaxial Transmission Line" (2019). Graduate Theses, Dissertations, and Problem Reports. 3933. https://researchrepository.wvu.edu/etd/3933

This Thesis is protected by copyright and/or related rights. It has been brought to you by the The Research Repository @ WVU with permission from the rights-holder(s). You are free to use this Thesis in any way that is permitted by the copyright and related rights legislation that applies to your use. For other uses you must obtain permission from the rights-holder(s) directly, unless additional rights are indicated by a Creative Commons license in the record and/ or on the work itself. This Thesis has been accepted for inclusion in WVU Graduate Theses, Dissertations, and Problem Reports collection by an authorized administrator of The Research Repository @ WVU. For more information, please contact researchrepository@mail.wvu.edu. 


\title{
Experimental Measurement of Dielectric Properties of Powdery Materials using a Coaxial Transmission Line
}

\author{
Robert Sean Tempke
}

\author{
Thesis submitted \\ to the Benjamin M. Statler College of \\ Engineering and Mineral Resources \\ at West Virginia University
}

in partial fulfillment of the requirements for the degree of

Master of Science

in

Mechanical Engineering

\author{
Terence Musho, Ph.D., Chair \\ Edward Sabolsky, Ph.D. \\ Christina Wildfire, Ph.D. \\ Department of Mechanical and Aerospace Engineering \\ Morgantown, West Virginia \\ May 2019
}

Keywords: Composite, Dielectric Constant, Microwave, Paraffin, Standardized Method, Dielectric Properties

Copyright 2019 Robert Sean Tempke 


\begin{abstract}
Experimental Measurement of Dielectric Properties of Powdery Materials using a Coaxial Transmission Line

Robert Tempke
\end{abstract}

This study proposes a standard methodology for coaxial dielectric property measurements of powdery materials $(1-10 \mathrm{GHz})$ using a coaxial transmission line. Four powdery materials with dielectric constants ranging from 3.5 to $70\left(\mathrm{SiO}_{2}, \mathrm{Al}_{2} \mathrm{O}_{3}, \mathrm{CeO}_{2}\right.$, and $\left.\mathrm{TiO}_{2}\right)$ were experimentally investigated at varying volume loading fractions in a paraffin mixture. A statistically significant number of paraffin heterogeneous-mixtures was synthesized for all dielectric powders. The dielectric properties of the constitutive materials were determined using appropriate mixture equations. The sensitivity of the equations dielectric prediction to volume loading is discussed with guidance on selecting the best mixing equation. It was determined that low volume loadings of less than $10 \%$ was ideal for a general case where little to nothing is known about the sample. If the general range of the dielectric constant is known different mixture equations were found to be optimal for specific ranges. Furthermore, there was not a single mixing equation that proved best over the entire range of dielectrics studied. To this end, a standard testing procedure for powdery materials is justified, with guidelines for selection of an appropriate mixture equation. The justification is based on the ratio of the dielectric constant of the powder to that of the paraffin. These findings provide a baseline procedure for determining dielectric properties of new powdery materials that have applications in the area of microwave catalysis. 
To my family the ones I was born with and the ones I chose.

Thank you to Rhiannon Schmitt for all the support and love you have shown me while I worked late into the night. 


\section{ACKNOWLEDGMENTS}

First and foremost, I would like to thank my advisor and mentor Dr. Terence D. Musho for all his support and guidance in leading me through my research. His guidance and advice were paramount to my success and growth over these last few years. I look forward to continuing out work together as we move forward on new and exciting projects.

I would also like to give special thanks to Dr. Christina Wildfire who mentored me and showed me the importance of experimental lab work. I made frequent use of her open door to ask her a constant barrage of questions which she tirelessly answered. If not for her I would still be in the lab trying to figure out what was going on.

A special thanks is required for all my colleagues at NETL, who despite some less than conventional motivational methods were of great help in all my research. One thing I would like to remember is donuts are not free. 
Table of Contents

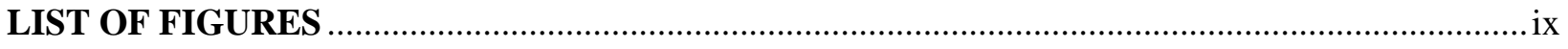

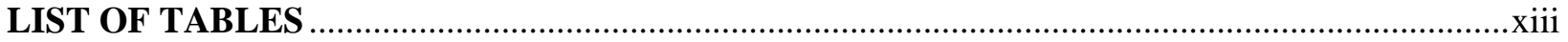

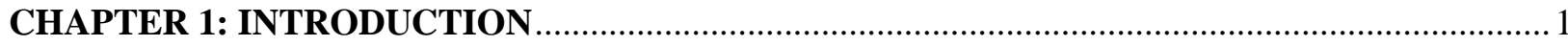

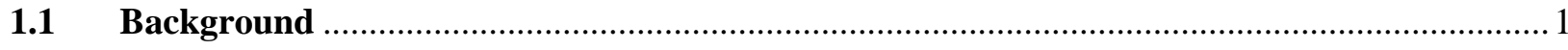

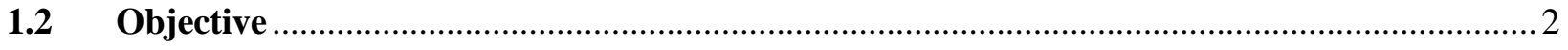

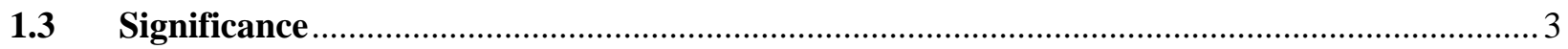

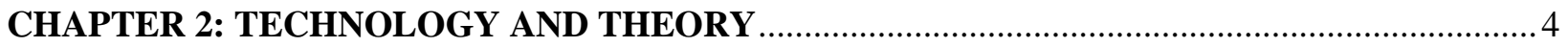

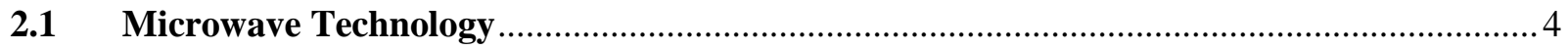

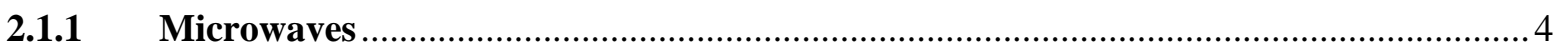

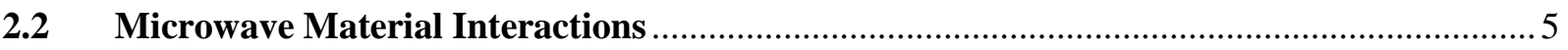

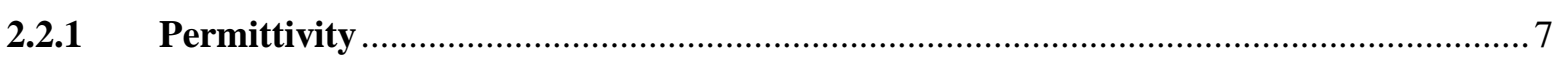

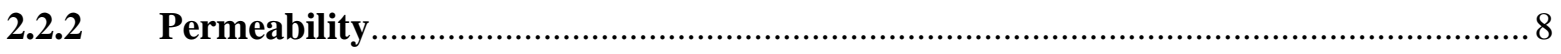

2.2.3 Microscopic, Local and Macroscopic Fields ......................................................... 9

2.2.4 Local Electromagnetic Fields in Materials ........................................................... 9

2.3 Measurement Models for Material Properties .............................................................. 10

2.3.1 Transmission/Reflection measurement techniques for lossy materials..................... 10

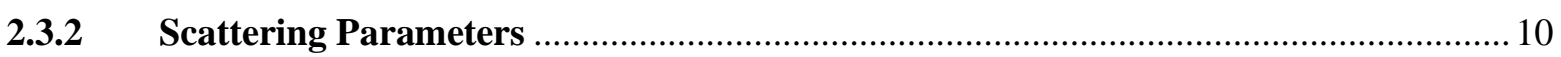

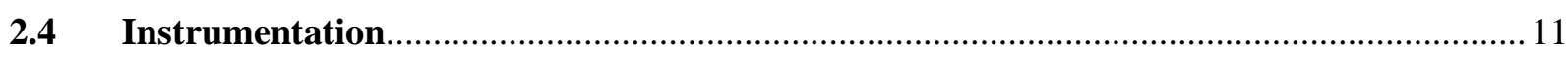

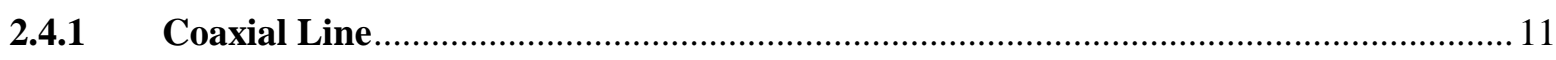

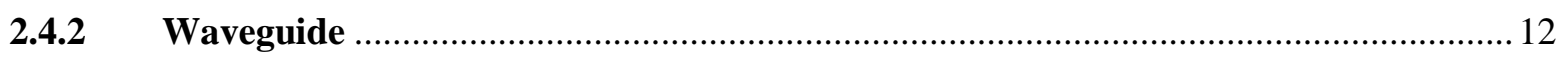

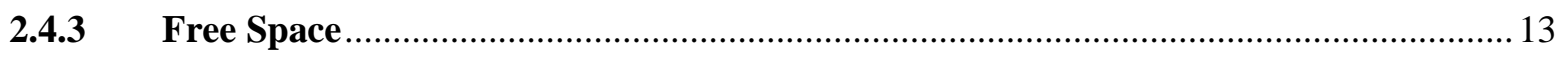

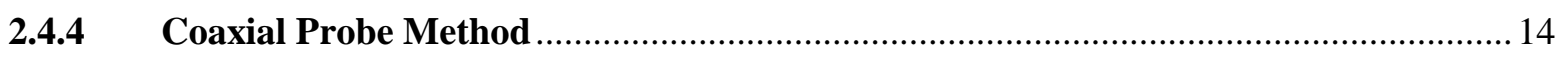

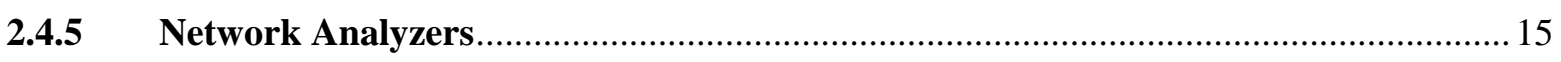

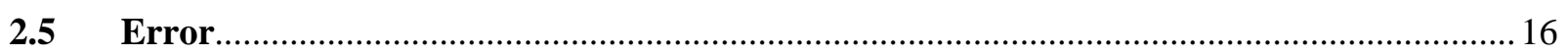

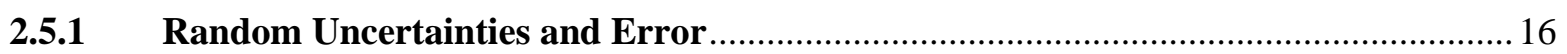

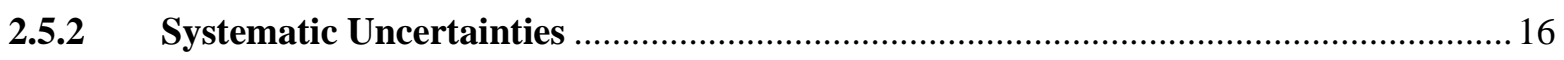

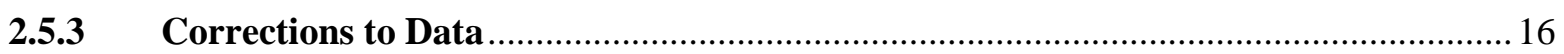

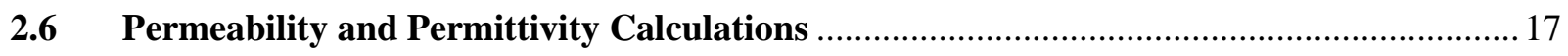

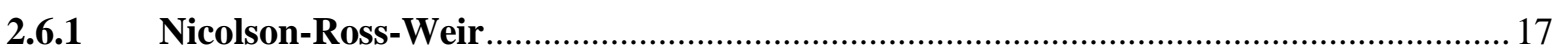

2.6.2 Nicolson-Ross-Weir Polynomial Method ............................................................... 17

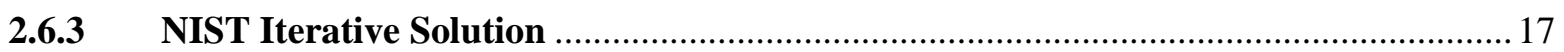

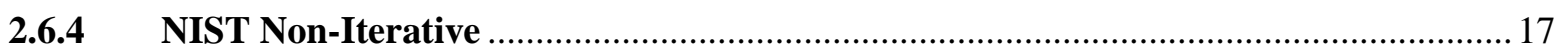

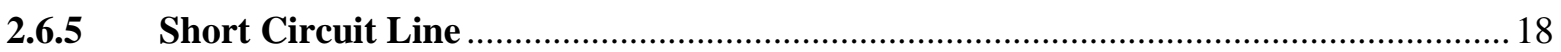




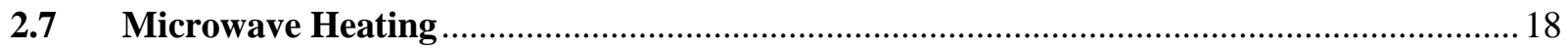

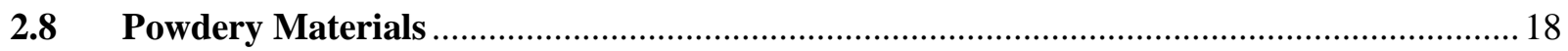

2.9 Specimen Preparations for Powdery Material ................................................................. 19

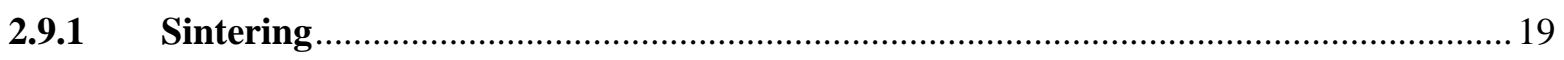

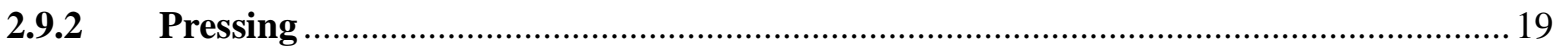

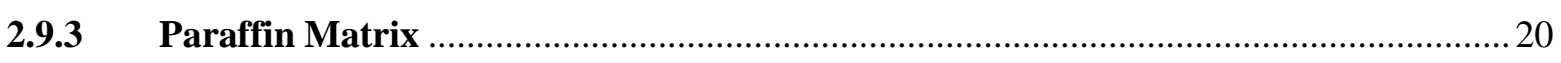

2.10 Mixture equations for Two Phase Homogenous Composites ............................................2

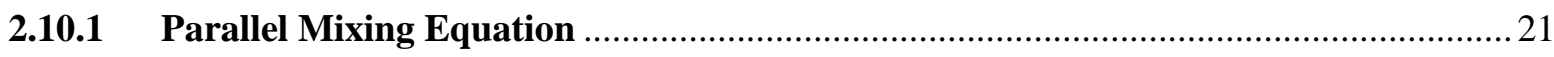

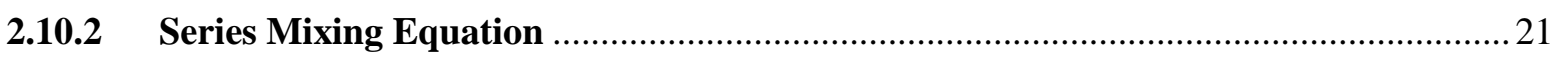

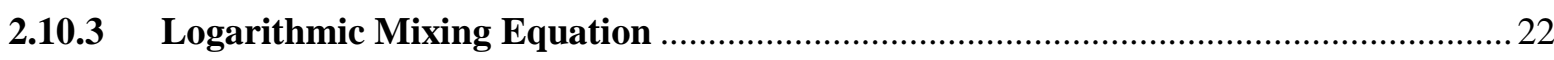

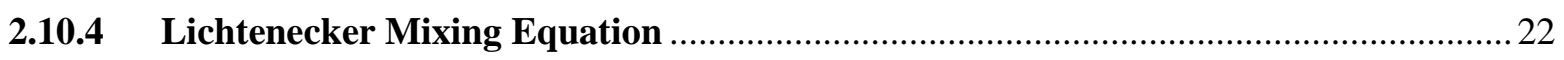

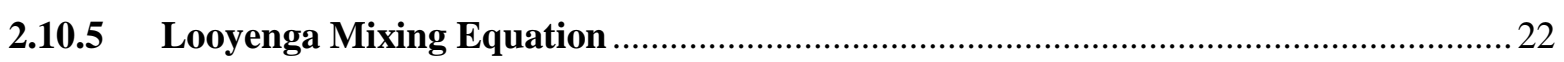

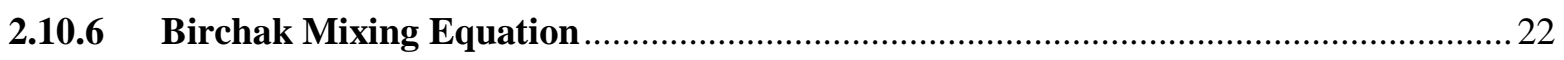

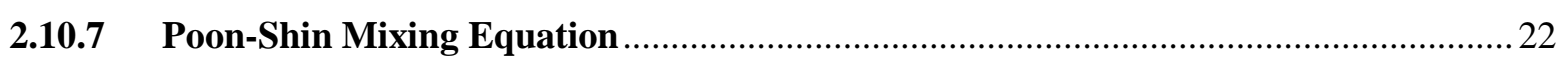

2.10.8 Effective Medium Theory Mixing Equation ............................................................... 23

2.10.9 Maxwell-Garnet Mixing Equation ............................................................................ 23

2.10.10 Jayasundere-Smith Mixing Equation.................................................................... 23

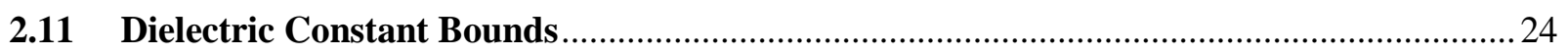

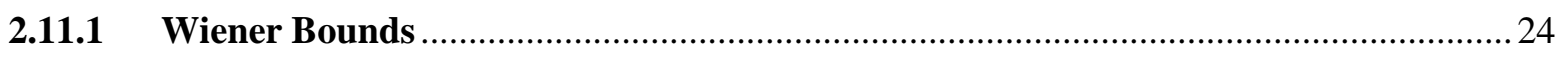

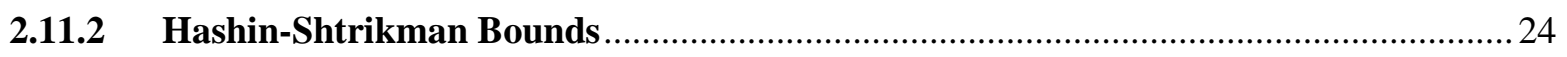

CHAPTER 3: EXPERIMENTAL TESING OF POWDERY MATERIALS ..................................26

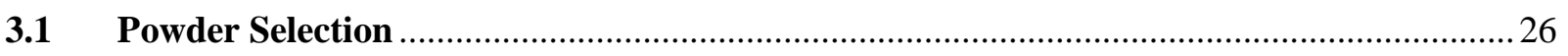

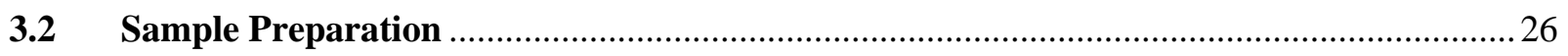

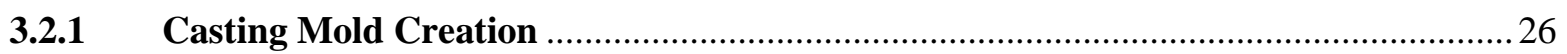

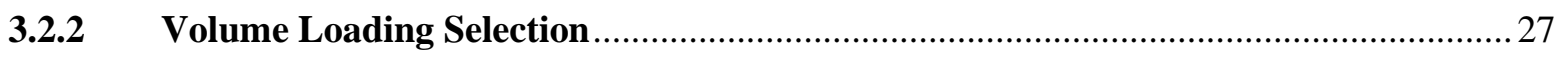

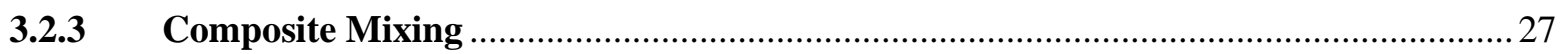

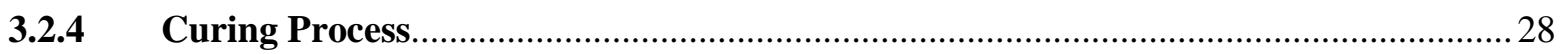

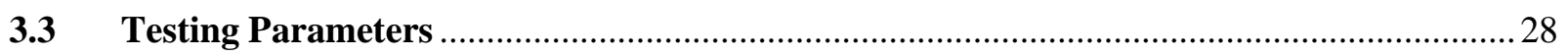

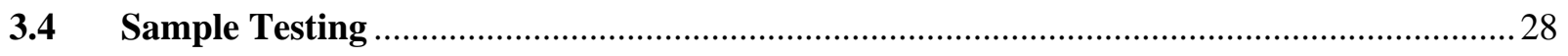

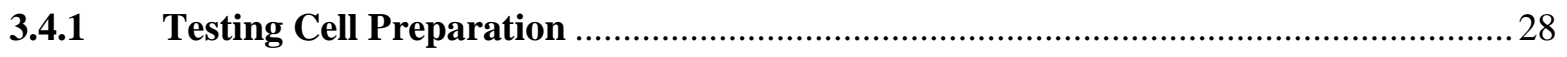

3.4.2 Sample Loading in Coaxial Test Cell ................................................................ 28

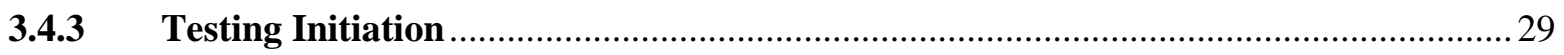

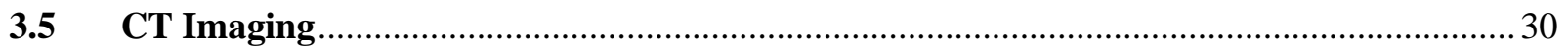

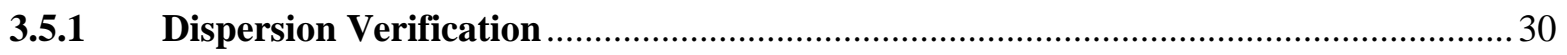

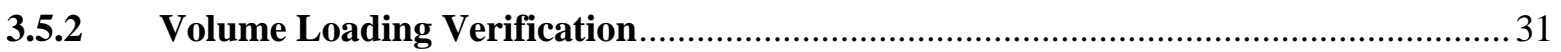




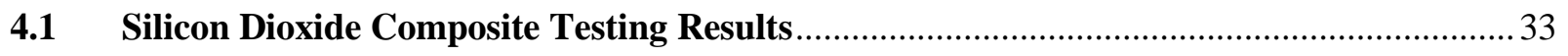

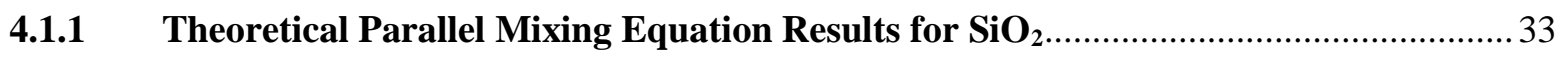

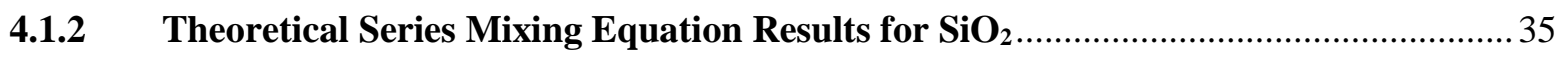

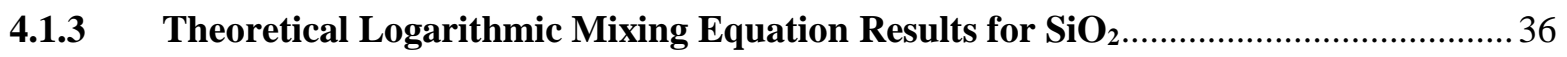

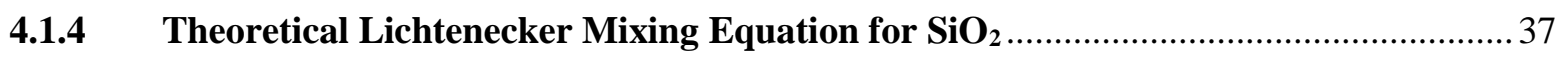

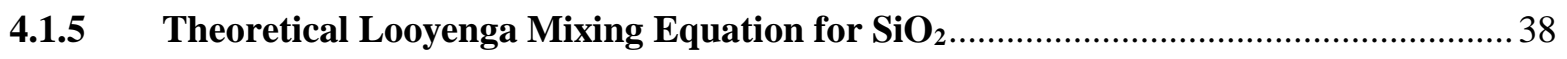

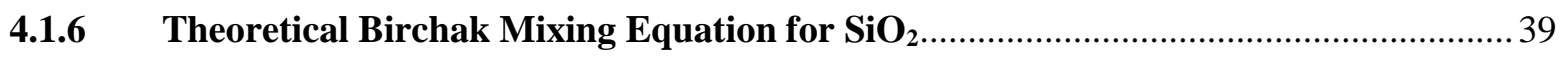

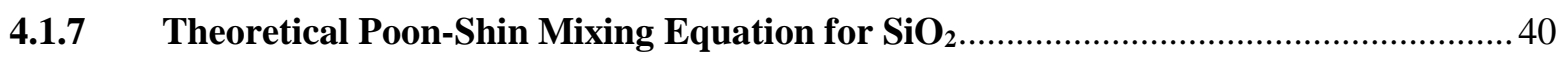

4.1.8 Theoretical Effective Medium Theory Mixing Equation for $\mathrm{SiO}_{2} \ldots \ldots \ldots \ldots \ldots \ldots \ldots \ldots . . . . . . . . . . . . . . .41$

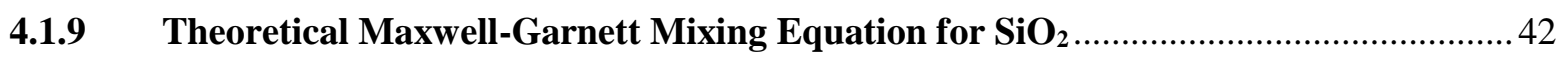

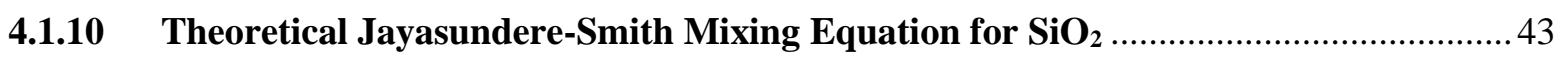

4.2 Aluminum Oxide Dioxide Composite Testing Results...................................................... 44

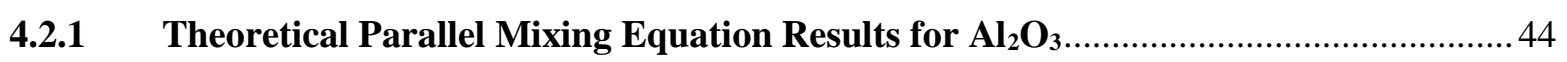

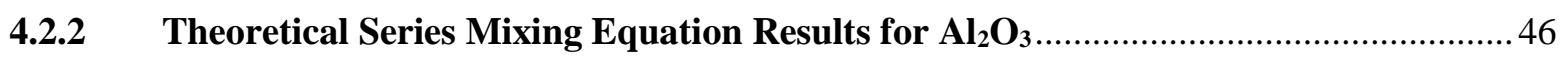

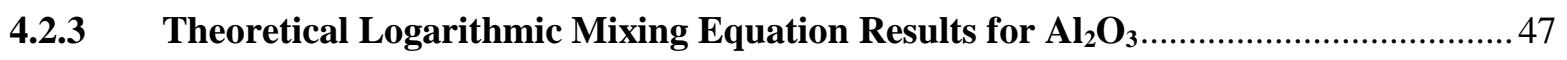

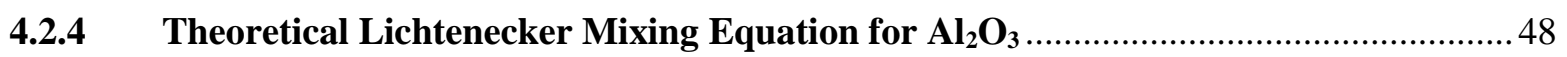

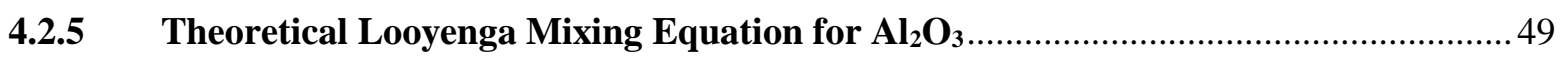

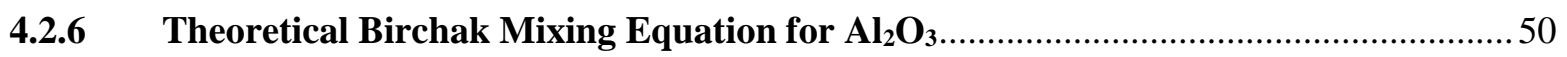

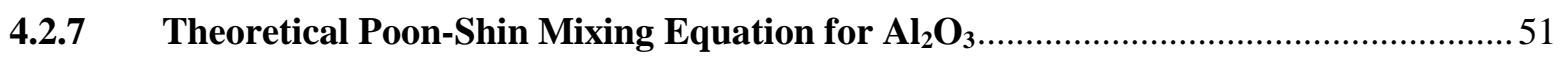

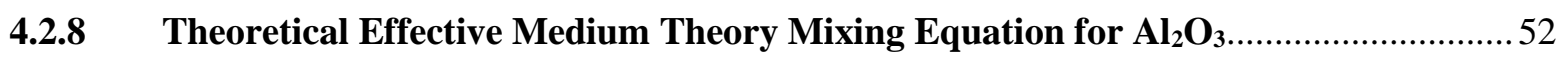

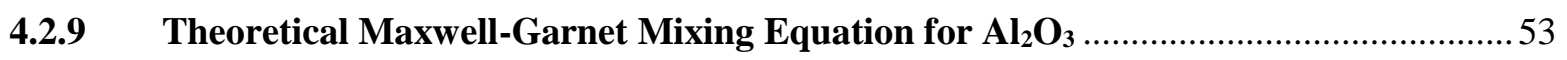

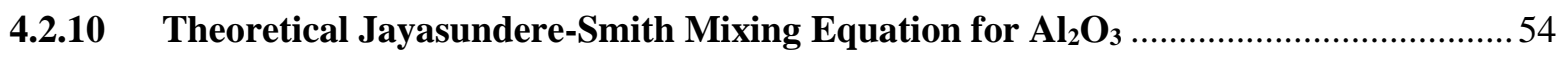

4.3 Cerium Dioxide Composite Testing Results ….............................................................5

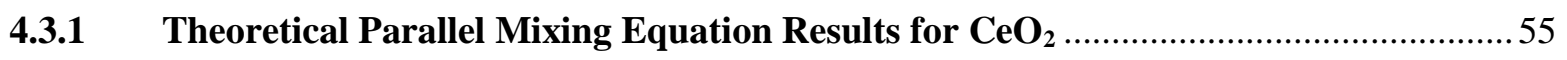

4.3.2 Theoretical Series Mixing Equation Results for $\mathrm{CeO}_{2}$.............................................57

4.3.3 Theoretical Logarithmic Mixing Equation Results for $\mathrm{CeO}_{2}$.....................................58

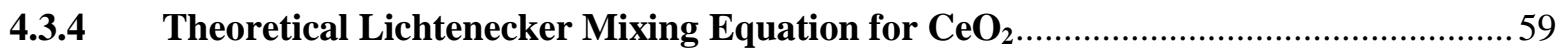

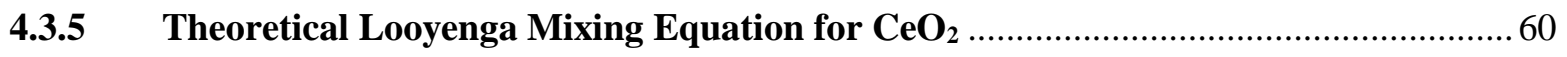

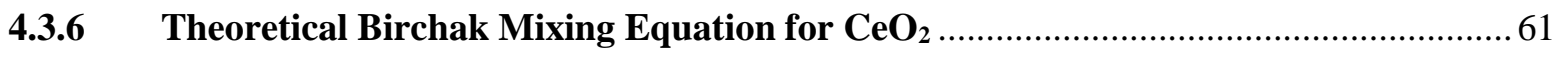

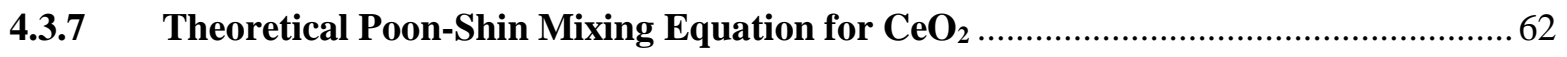

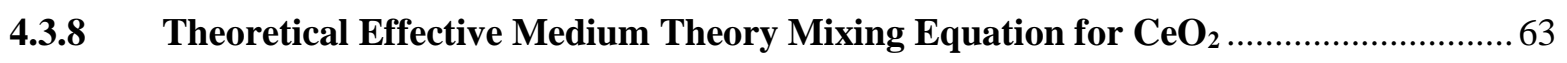

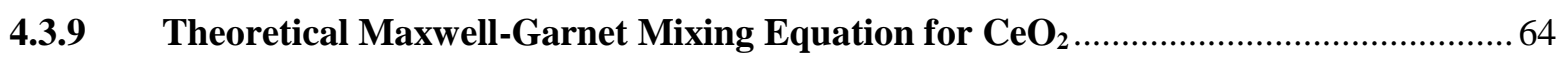

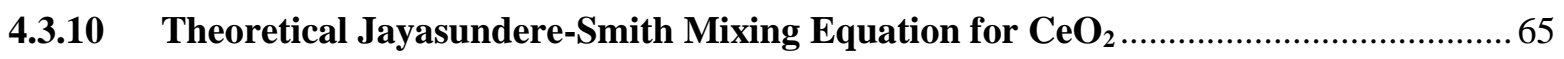

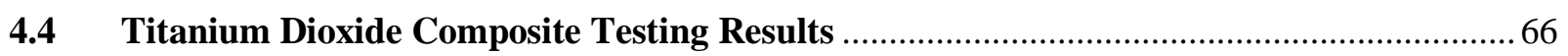




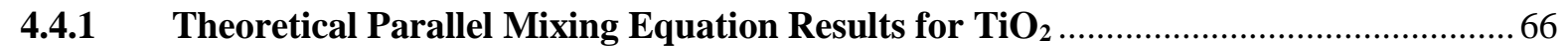

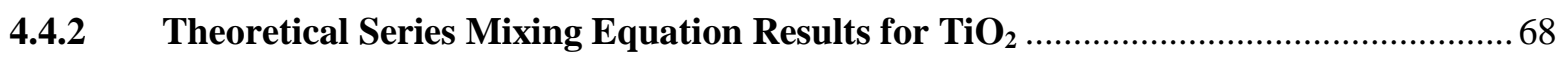

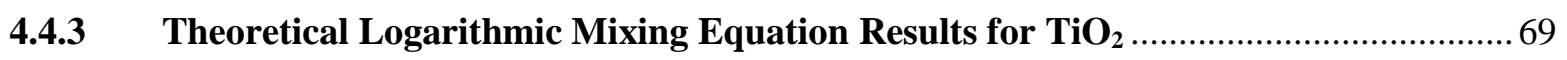

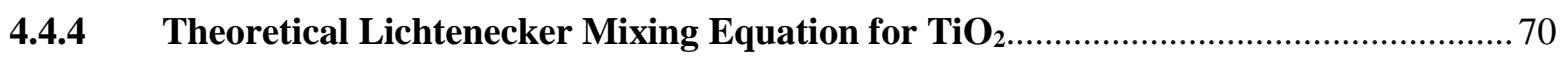

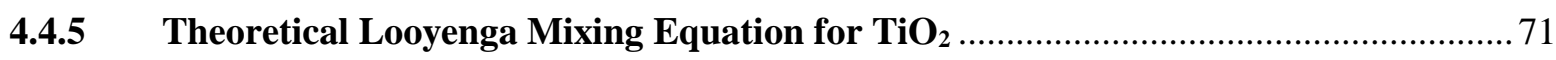

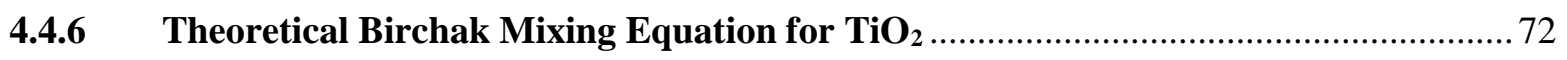

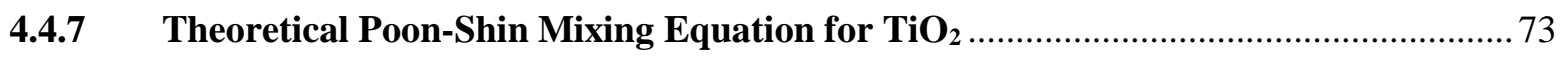

4.4.8 Theoretical Effective Medium Theory Mixing Equation for $\mathrm{TiO}_{2} \ldots \ldots \ldots \ldots \ldots \ldots \ldots \ldots \ldots . . . . . . . . . . . . . .74$

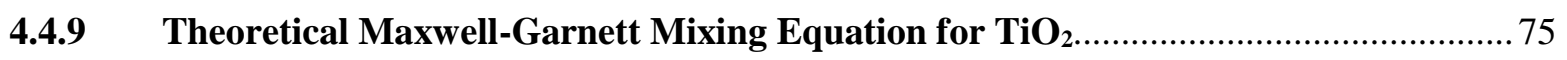

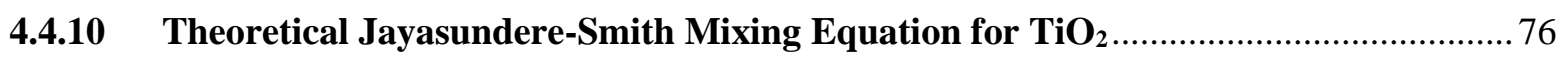

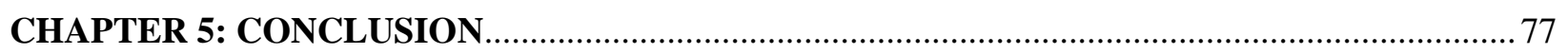

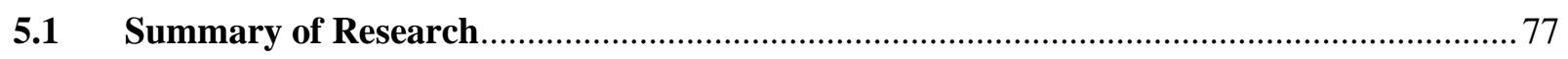

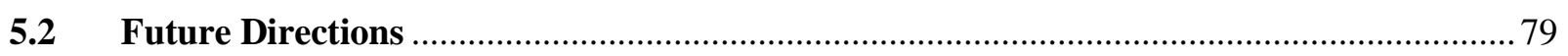

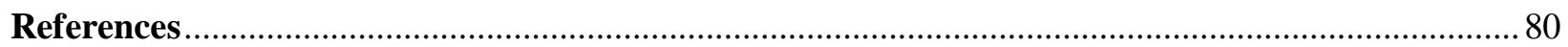




\section{LIST OF FIGURES}

Figure 2.1: Electromagnetic spectrum with a visualization of the visible spectrum shown as a subset of electromagnetic radiation. 4

Figure 2.2: An electromagnetic wave propagating in the $+\mathrm{z}$ direction through a homogenous, isotropic, dissipationless medium. The wave is linearly polarized, where the electric field is shown in blue and the magnetic field is shown in red. The electric field oscillates in the $\pm x$ direction while the magnetic field oscillates in the $\pm \mathrm{y}$ direction 5

Figure 2.3: a: electrical conducting material, b: insulating material, c: dielectric material ${ }^{16}{ }^{16} .7$

Figure 2.4: Dielectric permittivity spectrum over a wide range of frequencies. Various processes are labeled on the image: ionic and dipolar relaxation, atomic and electronic resonances at higher energies ${ }^{67}$....... 8

Figure 2.5 Single wave in a two-port electrical-element. Simple representation of a standard 2 port measurement for S-parameters ${ }^{31}$

Figure 2.6: Keysight Network high precision coaxial airline. Used to measure the scattering parameters and calculate the associated material properties. Example of a testable material is shown as the composite 34

Figure 2.7: Waveguides for use in vector network analyzer, shown are both a circular and rectangular waveguide ${ }^{68}$

Figure 2.8: Illustration of the Free space method during testing of material properties ${ }^{30}$ 14

Figure 2.9: Standard Keysight High Temperature Coaxial Probe, used to collect scattering parameters from a material so a VNA can calculate the material properties ${ }^{28}$ 15

Figure 2.10: Keysight Network Analyzer part of the vector network analyzer family of machinery. Used primarily to measure the scattering parameters and calculate the associated material properties. VNA's are used for many of the different techniques mentioned in the following sections ${ }^{34}$ 16

Figure 2.11: (a) Alumina pellet with individual grains, (b) well sintered pellet with defined cleavage plans at $1580^{\circ} \mathrm{C}^{48}$ 19

Figure 2.12: Effects of particle size on both hot isostatic pressing and cold isostatic pressing ${ }^{51}$ 20

Figure 2.13: The maximum effective permittivity for a given volume fraction of inclusions manifest if the inclusions are in the direction of the flux as shown on the left side. The minimum effective permittivity for a given volume fraction of inclusions corresponds to when the flux is forced to pass through the phase with lower permittivity ${ }^{56}$

Figure 2.14: Wiener bounds and Hashin-Shtrikman bounds for dielectric constant of a mixture with a dielectric inclusion of $10^{24}$ 
Figure 3.1: (A) 3-D rendering of casting mold for paraffin composite mixtures. The molds are reusable and provide consistency from batch to batch production. (B) The final 3D printed product with a composite mold attached for visualization

Figure 3.2: Paraffin-powder material composite plug loaded into the precision airline. The plug fills all the space between the center electrode and the outer electrode to ensure no airgaps exist.

Figure 3.3: VNA setup for high precision coaxial airline testing. Two port VNA with a $10 \mathrm{CM}$ testing line..... 30

Figure 3.4: Cross sectional view of an $\mathrm{Al}_{2} \mathrm{O}_{3}$ that allows for a visual confirmation of powder distribution within the paraffin matrix. The lines on the bottom are a consequence of beam hardening in the CT scanner ${ }^{66}$

Figure 3.5: 3D rendering from CT images of a $\mathrm{CeO} 230 \%$ volume loading composite. Reconstruction was preformed using FIJI and volume loading was verified using there built in software ${ }^{66}$

Figure 4.1: Theoretical Parallel Mixture equation for $\mathrm{SiO} 2$ mapped against experimental scatter plot data for four different volume loadings

Figure 4.2: Theoretical Series Mixture equation for $\mathrm{SiO} 2$ mapped against experimental scatter plot data for four different volume loadings 35

Figure 4.3: Theoretical Logarithmic Mixture equation for $\mathrm{SiO} 2$ mapped against experimental scatter plot data for four different volume loadings.

Figure 4.4: Theoretical Lichtenecker mixture equation for $\mathrm{SiO} 2$ mapped against experimental scatter plot data for four different volume loadings.

Figure 4.5: Theoretical Looyenga mixture equation for $\mathrm{SiO} 2$ mapped against experimental scatter plot data for four different volume loadings. 38

Figure 4.6: Theoretical Birchak mixture equation for $\mathrm{SiO} 2$ mapped against experimental scatter plot data for four different volume loadings.... 39

Figure 4.7: Theoretical Poon-Shin Mixture equation for $\mathrm{SiO} 2$ mapped against experimental scatter plot data for four different volume loadings.

Figure 4.8: Theoretical EMT mixture equation for $\mathrm{SiO} 2$ mapped against experimental scatter plot data for four different volume loadings .

Figure 4.9: Theoretical Maxwell-Garnett mixture equation for $\mathrm{SiO} 2$ mapped against experimental scatter plot data for four different volume loadings. 42

Figure 4.10: Theoretical Jayasundere-Smith mixture equation for $\mathrm{SiO} 2$ mapped against experimental scatter plot data for four different volume loadings. 
Figure 4.11: Theoretical Parallel Mixture equation for $\mathrm{A} 12 \mathrm{O} 3$ mapped against experimental scatter plot data for four different volume loadings.

Figure 4.12: Theoretical Series Mixture equation for Al2O3 mapped against experimental scatter plot data for four different volume loadings. 46

Figure 4.13: Theoretical Logarithmic Mixture equation for $\mathrm{Al} 2 \mathrm{O} 3$ mapped against experimental scatter plot data for four different volume loadings.

Figure 4.14: Theoretical Lichtenecker mixture equation for Al2O3 mapped against experimental scatter plot data for four different volume loadings.

Figure 4.15: Theoretical Looyenga mixture equation for $\mathrm{A} 12 \mathrm{O} 3$ mapped against experimental scatter plot data for four different volume loadings.

Figure 4.16: Theoretical Birchak mixture equation for $\mathrm{A} 12 \mathrm{O} 3$ mapped against experimental scatter plot data for four different volume loadings. 50

Figure 4.17: Theoretical Poon-Shin Mixture equation for A12O3 mapped against experimental scatter plot data for four different volume loadings.

Figure 4.18: Theoretical EMT mixture equation for A12O3mapped against experimental scatter plot data for four different volume loadings

Figure 4.19: Theoretical Maxwell-Garnett mixture equation for Al2O3mapped against experimental scatter plot data for four different volume loadings.

Figure 4.20: Theoretical Jayasundere-Smith mixture equation for Al2O3mapped against experimental scatter plot data for four different volume loadings....

Figure 4.21: Theoretical Parallel Mixture equation for $\mathrm{CeO}_{2}$ mapped against experimental scatter plot data for four different volume loadings.

Figure 4.22: Theoretical Series Mixture equation for $\mathrm{CeO}_{2}$ mapped against experimental scatter plot data for four different volume loadings....

Figure 4.23: Theoretical Logarithmic Mixture equation for $\mathrm{CeO}_{2}$ mapped against experimental scatter plot data for four different volume loadings.

Figure 4.24: Theoretical Lichtenecker mixture equation for $\mathrm{CeO}_{2}$ mapped against experimental scatter plot data for four different volume loadings.

Figure 4.25: Theoretical Looyenga mixture equation for $\mathrm{CeO}_{2}$ mapped against experimental scatter plot data for four different volume loadings

Figure 4.26: Theoretical Birchak mixture equation for $\mathrm{CeO}_{2}$ mapped against experimental scatter plot data for four different volume loadings.

Figure 4.27: Theoretical Poon-Shin Mixture equation for $\mathrm{CeO}_{2}$ mapped against experimental scatter plot data for four different volume loadings. 
Figure 4.28: Theoretical EMT mixture equation for $\mathrm{CeO}_{2}$ mapped against experimental scatter plot data for four different volume loadings

Figure 4.29: Theoretical Maxwell-Garnett mixture equation for $\mathrm{CeO}_{2}$ mapped against experimental scatter plot data for four different volume loadings 64

Figure 4.30: Theoretical Jayasundere-Smith mixture equation for $\mathrm{CeO}_{2}$ mapped against experimental scatter plot data for four different volume loadings

Figure 4.31: Theoretical Parallel Mixture equation for $\mathrm{CeO}_{2}$ mapped against experimental scatter plot data for four different volume loadings. 67

Figure 4.32: Theoretical Series Mixture equation for $\mathrm{CeO}_{2}$ mapped against experimental scatter plot data for four different volume loadings 68

Figure 4.33: Theoretical Logarithmic Mixture equation for $\mathrm{CeO}_{2}$ mapped against experimental scatter plot data for four different volume loadings.

Figure 4.34: Theoretical Lichtenecker mixture equation for $\mathrm{CeO}_{2}$ mapped against experimental scatter plot data for four different volume loadings. 70

Figure 4.35: Theoretical Looyenga mixture equation for $\mathrm{CeO}_{2}$ mapped against experimental scatter plot data for four different volume loadings.

Figure 4.36: Theoretical Birchak mixture equation for $\mathrm{CeO}_{2}$ mapped against experimental scatter plot data for four different volume loadings.

Figure 4.37: Theoretical Poon-Shin Mixture equation for $\mathrm{CeO}_{2}$ mapped against experimental scatter plot data for four different volume loadings.

Figure 4.38: Theoretical EMT mixture equation for $\mathrm{CeO}_{2}$ mapped against experimental scatter plot data for four different volume loadings 74

Figure 4.39: Theoretical Maxwell-Garnett mixture equation for $\mathrm{CeO}_{2}$ mapped against experimental scatter plot data for four different volume loadings.... 75

Figure 4.40: Theoretical Jayasundere-Smith mixture equation for $\mathrm{CeO}_{2}$ mapped against experimental scatter plot data for four different volume loadings..... 76 


\section{LIST OF TABLES}

Table 3.1: Powders used in the study to determine the accuracy of the mixture equations for twophase homogenous composites. Associated relevant information such as the supplier, density and

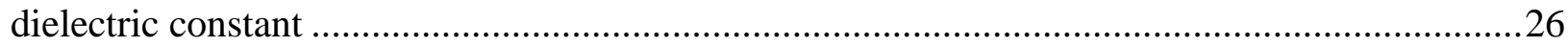

Table 4.1: Absolute Percent Error for $\mathrm{SiO}_{2}$ composites using Parallel mixing equation .............34

Table 4.2: Absolute Percent Error for $\mathrm{SiO}_{2}$ composites using Series mixing equation.................35

Table 4.3: Absolute Percent Error for $\mathrm{SiO}_{2}$ composites using Logarithmic mixing equation ......36

Table 4.4: Absolute Percent Error for $\mathrm{SiO}_{2}$ composites using Lichtenecker mixing equation....37

Table 4.5: Absolute Percent Error for $\mathrm{SiO}_{2}$ composites using Looyenga mixing equation..........38

Table 4.6: Absolute Percent Error for $\mathrm{SiO}_{2}$ composites using Birchak mixing equation .............39

Table 4.7: Absolute Percent Error for $\mathrm{SiO}_{2}$ composites using Poon-Shin mixing equation.........40

Table 4.8: Absolute Percent Error for $\mathrm{SiO}_{2}$ composites using EMT mixing equation .................41

Table 4.9: Absolute Percent Error for $\mathrm{SiO}_{2}$ composites using Maxwell-Garnett mixing equation .42

Table 4.10: Absolute Percent Error for $\mathrm{SiO}_{2}$ composites using Jayasundere-Smith mixing equation .43

Table 4.11: Absolute Percent Error for $\mathrm{Al}_{2} \mathrm{O}_{3}$ composites using Parallel mixing equation .........44

Table 4.12: Absolute Percent Error for $\mathrm{Al}_{2} \mathrm{O}_{3}$ composites using Series mixing equation............46

Table 4.13: Absolute Percent Error for $\mathrm{Al}_{2} \mathrm{O}_{3}$ composites using Logarithmic mixing equation .47

Table 4.14: Absolute Percent Error for $\mathrm{Al}_{2} \mathrm{O}_{3}$ composites using Lichtenecker mixing equation

Table 4.15: Absolute Percent Error for $\mathrm{Al}_{2} \mathrm{O}_{3}$ composites using Looyenga mixing equation.....49

Table 4.16: Absolute Percent Error for $\mathrm{Al}_{2} \mathrm{O}_{3}$ composites using Birchak mixing equation.........50

Table 4.17: Absolute Percent Error for $\mathrm{Al}_{2} \mathrm{O}_{3}$ composites using Poon-Shin mixing equation....51

Table 4.18: Absolute Percent Error for $\mathrm{Al}_{2} \mathrm{O}_{3}$ composites using EMT mixing equation .............52

Table 4.19: Absolute Percent Error for $\mathrm{Al}_{2} \mathrm{O}_{3}$ composites using Maxwell-Garnett mixing equation .53

Table 4.20: Absolute Percent Error for $\mathrm{Al}_{2} \mathrm{O}_{3}$ composites using Jayasundere-Smith mixing equation .54 
Table 4.21: Absolute Percent Error for $\mathrm{CeO}_{2}$ composites using Parallel mixing equation .........56

Table 4.22: Absolute Percent Error for $\mathrm{CeO}_{2}$ composites using Series mixing equation............57

Table 4.23: Absolute Percent Error for $\mathrm{CeO}_{2}$ composites using Logarithmic mixing equation ..58

Table 4.24: Absolute Percent Error for $\mathrm{CeO}_{2}$ composites using Lichtenecker mixing equation .59

Table 4.25: Absolute Percent Error for $\mathrm{CeO}_{2}$ composites using Looyenga mixing equation .....60

Table 4.26: Absolute Percent Error for $\mathrm{CeO}_{2}$ composites using Birchak mixing equation.........61

Table 4.27: Absolute Percent Error for $\mathrm{CeO}_{2}$ composites using Poon-Shin mixing equation.....62

Table 4.28: Absolute Percent Error for $\mathrm{CeO}_{2}$ composites using EMT mixing equation ............63

Table 4.29: Absolute Percent Error for $\mathrm{CeO}_{2}$ composites using Maxwell-Garnett mixing equation .64

Table 4.30: Absolute Percent Error for $\mathrm{CeO}_{2}$ composites using Jayasundere-Smith mixing equation...... .65

Table 4.31: Absolute Percent Error for $\mathrm{TiO}_{2}$ composites using Parallel mixing equation ..........67

Table 4.32: Absolute Percent Error for $\mathrm{TiO}_{2}$ composites using Series mixing equation............68

Table 4.33: Absolute Percent Error for $\mathrm{TiO}_{2}$ composites using Logarithmic mixing equation ...69

Table 4.34: Absolute Percent Error for $\mathrm{TiO}_{2}$ composites using Lichtenecker mixing equation .70

Table 4.35: Absolute Percent Error for $\mathrm{TiO}_{2}$ composites using Looyenga mixing equation ......71

Table 4.36: Absolute Percent Error for $\mathrm{TiO}_{2}$ composites using Birchak mixing equation..........72

Table 4.37: Absolute Percent Error for $\mathrm{TiO}_{2}$ composites using Poon-Shin mixing equation .....73

Table 4.38: Absolute Percent Error for $\mathrm{TiO}_{2}$ composites using EMT mixing equation ..............74

Table 4.39: Absolute Percent Error for $\mathrm{TiO}_{2}$ composites using Maxwell-Garnett mixing equation .75

Table 4.40: Absolute Percent Error for $\mathrm{TiO}_{2}$ composites using Jayasundere-Smith mixing equation .76

Table 5.1: The best mixing equation for each powdery material studied and the associated averaged error over the volume fractions studied between the equation and the experimental values. The EMT mixing equation is recommended over the Maxwell-Garnett for simplicity despite a statistically insignificant improvement of error when using the latter equation............78

Table 5.2: Suggested mixing equation based on the ratio of powder's dielectric properties to matrix material's dielectric properties .78 


\section{CHAPTER 1: INTRODUCTION}

\subsection{Background}

The experimental characterization of how electromagnetic (EM) waves interact with powdery materials is critical to new devices and process developments at $\mathrm{GHz}$ frequencies. While the physics of this wave-matter interaction at microwave wavelengths has been well established, the experimental measurement technique to derive the properties is often subject to considerable random error. These random errors are typically a result of inconsistency of the testing procedural method and large uncertainty in the mathematical approach used to determine the constitutive dielectric properties from a mixture. Moreover, there will be variability in the dielectric constant of a material between measurements even using the same sample preparation method, dependent upon the dipole-dipole interaction within the sample ${ }^{1}$. Currently in literature there are no guidelines or best practice methodologies that allow researchers to consistently and accurately measure the dielectric constant of powdery materials. In considering all the aforementioned uncertainties in the dielectric measurement of powdery materials this paper outlines and justifies several best practice guidelines for determined the dielectrics of powdery materials.

One of the targeted applications of these powdery materials is for the transformation of chemical processes at microwave frequencies that have the potential to achieve new processing windows. With new processing windows comes the potential for new product distribution at higher conversion efficiencies, permitting new modular reactor designs. Often these chemical processes, require the use of a catalytic material that have tailored microwave absorption properties. In tailoring these microwave sensitive catalysts there are several physical attributes that contribute to how well these particles absorb EM radiation; the particle shape, size, electrical conductivity, and heterogeneity all influence the bulk dielectric properties ${ }^{2}$. Moreover, powdery materials exemplify a majority of these problematic attributes with irregular shape and widely varying material properties.

The complexity of these catalyst materials leads to inaccurate dielectric constant measurements which is extrapolated by the difficulties associated with the different measurement methods. There are multiple measurement techniques, such as rectangular free-space waveguide, open-ended probe, and coaxial precision airline that are used commonly in literature to measure the dielectric properties of materials ${ }^{3,4,5}$. The coaxial precision airline is the preferred method as it provides a precise measurement (low random error) of the transmission lines, which can readily be transformed into a complex dielectric constant. This study will focus on the use of a coaxial airline method with non-magnetic materials.

A brief survey of the current literature reveals several preparation methods used to measure powdery material in a high precision coaxial airline. These methods include sintering pellets of powdery materials and die pressing powders into pellets. Each of these methods come with their own difficulties and drawbacks, resulting in inconsistency of reported values. Air inclusion in pure powder testing is one of the greatest challenges; no matter how packed a powder, there will be air present in the plug creating increased variability during testing ${ }^{6}$. While sintering powders is a long complex process requiring extensive equipment and can often be time consuming. This coupled 
with bulk changes to the materials dielectric properties and density make it a less than ideal method for the testing of complex powders ${ }^{7,8}$. A better approach that retains the morphology of the powders is to cast the sample into a paraffin matrix. In addition to retaining the morphology, this method is less expensive, easier, and faster than sintering pellets. Moreover, it avoids any concern with transformation of the materials crystalline phase by post-heating or partial annealing of the sample during sintering. This makes it the preferred method, which is corroborated by many other studies in the literature.

\subsection{Objective}

The main objective of this study is to create a quick, easy, and cheap methodology for the dielectric testing of powdery materials in the frequency range of $1-10 \mathrm{GHz}$ that is accurate and repeatable. This study looks to use the paraffin composite method to test the powders dielectric constant without the need for extensive preprocessing and to avoid any changes to the structure of the powdery materials. Casting the powder in paraffin adds an additional phase to the transmission line measurement making it a composite. There are several mathematical expressions that can be used to calculate the composite properties of homogenous mixtures and can then be solved using the inverse of the mixing equation for the powder's dielectric properties. Though this is a wellcited practice, there is a lack of information on the procedural method of how to process these paraffin composites and select the correct mixing equation to achieve a high precision, low random error result ${ }^{9,10,11}$.

In order to provide clarity on this problem the study focused on two aims. The first aim was to determine if there is an ideal volume loading of powdery material into the paraffin matrix. This aim was achieved by analyzing the accuracy of 10 common two-phase mixture equations in calculating the dielectric constant of different volume loadings, using powdery materials of known dielectric constants. A look at the absolute percent error between the predictive values of the mixture equations and the measured dielectric constant of a composite can reveal what volume loadings range is more accurate for all mixing equations.

The second aim was to determine which equation was most accurate for a specific range of dielectric constants. Four different powdery materials with known frequency independent (in the microwave range) dielectric constants ranging from 2-100 were analyzed at four different volume loadings and analyzed with the same 10 mixing equations. Once again, the absolute percent error of each equation was calculated based on the output of the equations and the measured dielectric constant of the composite. This allows for a conclusion on which equation to use if the general range of the dielectric constant of the powder is known. 


\subsection{Significance}

The lack of a standard method in testing powdery materials is leading to an overabundance of studies being published that can't be compared to one another or researchers are incorrectly comparing studies that are fundamentally different. This study will provide researchers a standardized method of material preparation and measurement of a powder's dielectric properties that will decrease measurement error and provide a platform for research comparison. It will also lead to more accurate measurements of complex materials such as magnetic, paramagnetic, and heterogeneous/mixed-phase powders with a simple preparation method that will not alter the morphology of the material. 


\section{CHAPTER 2: TECHNOLOGY AND THEORY}

\subsection{Microwave Technology}

\subsubsection{Microwaves}

Microwaves are a form of electromagnetic radiation with a varying range of wavelengths from $3 \mathrm{~m}$ to $3 \mathrm{~mm}$ possessing frequencies of $1 \mathrm{GHz}$ to $100 \mathrm{GHz}$. Microwaves unlike radio frequencies travel by line of sight rather than as ground waves or as reflections from the ionosphere $^{12}$. Microwaves fall within the inferred and radio waves in the electromagnetic spectrum which is shown in Figure 2.13.

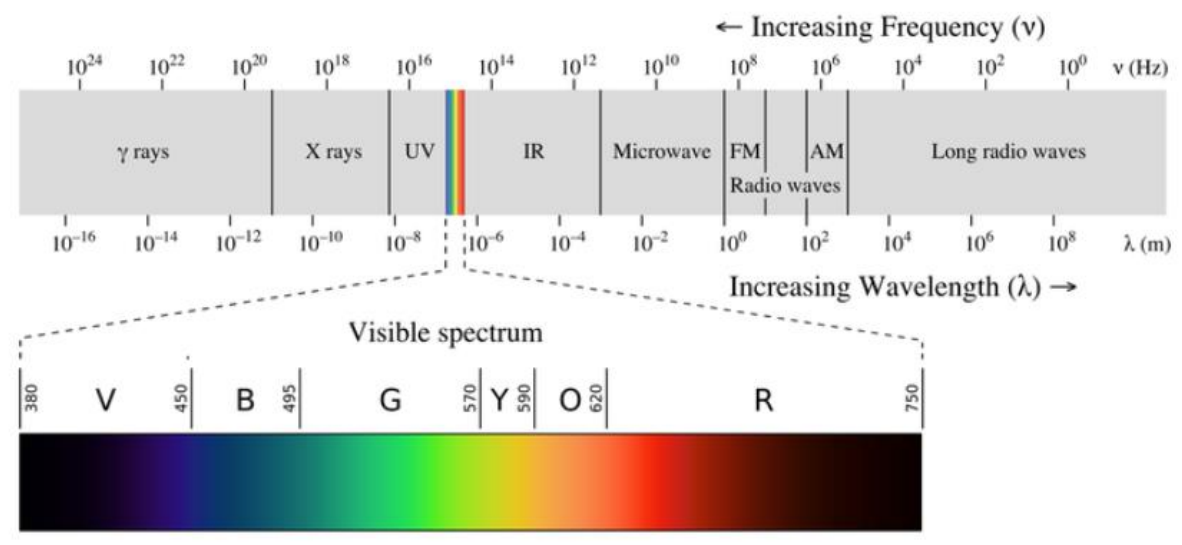

Figure 2.1: Electromagnetic spectrum with a visualization of the visible spectrum shown as a subset of electromagnetic radiation ${ }^{13}$.

Microwaves are used in a wide variety of applications such as point-to-point communication links, wireless networks, microwave radio relay networks, radar, medical diathermy, cancer treatments, remote sensing, satellite communication, spacecraft communication, radio astronomy, spectroscopy, industrial heating, collision avoidance systems, particle accelerators, garage door openers and keyless entry systems, and for cooking food in microwave ovens $^{14}$.

Microwaves consist of electromagnetic waves made up of two components, an electric field and a magnetic field. Microwaves are synchronized oscillations of the electric and magnetic fields both of which propagate at the speed of light. These two waves are commonly perpendicular to one another and to the direction of the energy, with this perpendicular wave propagation forming a transverse wave, this is shown in Figure 2.2 $2^{13}$. 


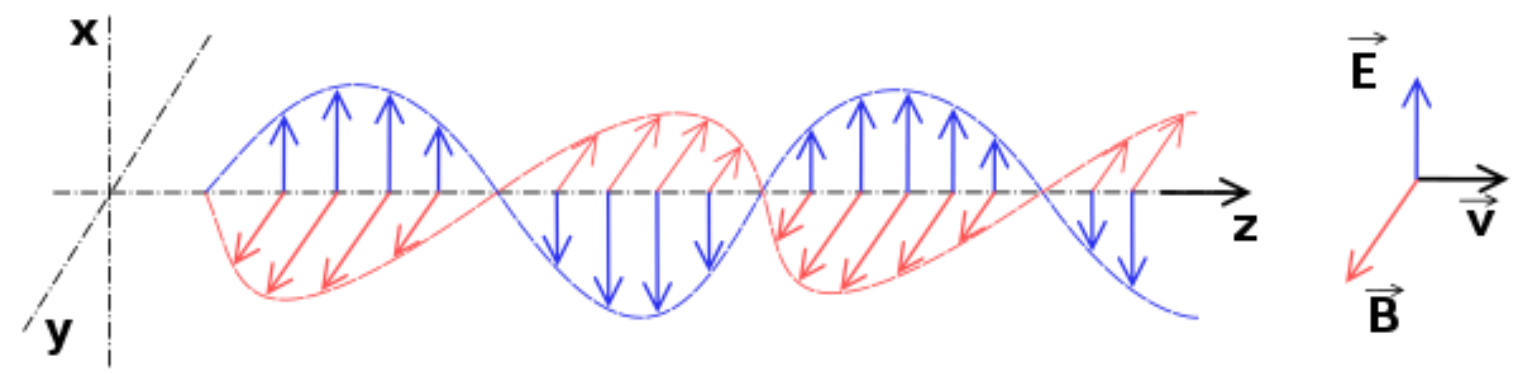

Figure 2.2 An electromagnetic wave propagating in the $+\mathrm{z}$ direction through a homogenous, isotropic, dissipationless medium. The wave is linearly polarized, where the electric field is shown in blue and the magnetic field is shown in red. The electric field oscillates in the $\pm \mathrm{x}$ direction while the magnetic field oscillates in the $\pm \mathrm{y}$ direction ${ }^{13}$.

Microwaves posses several unique characteristics and advantages over what can be seen at other wavelengths. These electromagnetic waves drastically reduce the time of heat conduction in a sample by directly heating the material ${ }^{15}$. These quick heating rates have been used in a variety of applications used in everyday life. Especially important for this study is the use of microwaves in the fields of organic chemistry, catalytic chemistry, inorganic material chemistry and analytical chemistry. Microwaves are especially useful in these fields over conventional heating as they drive regioselectivity, regular radical reaction, molecule orientation, high crystallization, anisotropic crystal, specific solid-phase diffusion, and strong reducing reaction ${ }^{15}$. The formation of temperature gradients at the microscale when using microwaves for catalyzed reactions if controlled would allow for huge advances in the field of catalytic chemistry. Microwaves are a type of non-ionizing radiation meaning that they do not contain enough energy to ionize or change substances.

\subsection{Microwave Material Interactions}

High-frequency electromagnetic waves effect solid materials in a multitude of ways depending on their different material classifications. Most materials used in microwave applications are designed to either pass a conduction current or prevent its flow as completely as possible. Conductors reflect microwaves from their surface without being effectively heated by the microwave. The electric field generated moves electrons freely from the surface of the material thereby heating the material via the resistivity of the heating material ${ }^{16,17}$. Conductive material can be regarded as a nonconducting dielectric with resistance in parallel. This allows for the microwaves to reflect off the material without absorption, losses, or heat generation. An illustrative example of this is shown in Figure 2.3a ${ }^{17}$.

Dielectric materials are characterized as materials that have changeable dipole interactions which result in heat generation ${ }^{17}$. The passage of microwave radiation through the medium generates absorption and heat generation throughout. ${ }^{16,16}$. Electromagnetic waves can be applied to heat dielectric materials by applying the electric field to induce polarization of the charges within the material being heated. The polarization cannot match the rapid reversals of the electric 
field and thus induces the heating of the irradiated media. It can also result in dipolar moments, which are merely localized reorganization of polar molecules. The magnetic component of electromagnetic waves introduces magnetic moments into the material. This local reorganization of linked and free charges is what is commonly known as the polarization phenomena. The polarization phenomena have two main points, the storage of electromagnetic energy within the irradiated medium and the conversation of thermal energy in relation to the frequency of the electromagnetic stimulation ${ }^{18,19,16}$. An illustrative example of a dielectric material is shown in Figure 2.3c.

The reorganization of linked and free charges is the physical origin of polarization phenomena which is clearly explain using quantum theory. The interaction between an electric and or magnetic field and a dipole can be explain using quantum theory. Weak coupling between dipole and electric field lead to no quantified orientations existing. Dipoles are typically associated with chemical bonds, and movement of the dipole induces a correlative motion in the molecular bonds. The motion of the magnetic moment is independent of this molecular motion ${ }^{1916}$.

The physical orientation of polarization can be expressed by the quantity $\vec{P}$ which gives the contribution of matter with regards to that of a vacuum. The electric field and the polarization are linked with the Maxwell's equations. The displacement and the electric field can be expressed as $\vec{D}$ and $\vec{E}$ respectively, their relationship can be seen in Equation one. The dielectric permittivity is the ratio of the electric displacement to the electric field ${ }^{19,16}$.

$$
\vec{D}=\overrightarrow{\bar{\varepsilon} E}
$$

The contribution of matter to polarization can be give as $\vec{P}$ and the dielectric medium can be characterized by $\bar{\varepsilon}$. $\vec{P}$ describes a polarization process relating to the response of dipoles and charges applied to the field. The relationships between these different fields is expressed in Equation two ${ }^{15,21}$.

$$
\vec{D}=\vec{\varepsilon} E=\varepsilon_{0} \vec{E}+\vec{P}
$$

Insulating materials allow microwaves to penetrate the material without absorption, losses or heat generation. The electronic reorientation or distortions of the induced and/or permanent dipole can result in heat generation within the material ${ }^{16,17,22}$. The material has the ability to polarize within the electric field generated by the microwave. A schematic of an insulating material is shown in Figure 2.3b with some examples of insulators being quartz glass, porcelain, ceramics, Teflon exc. 

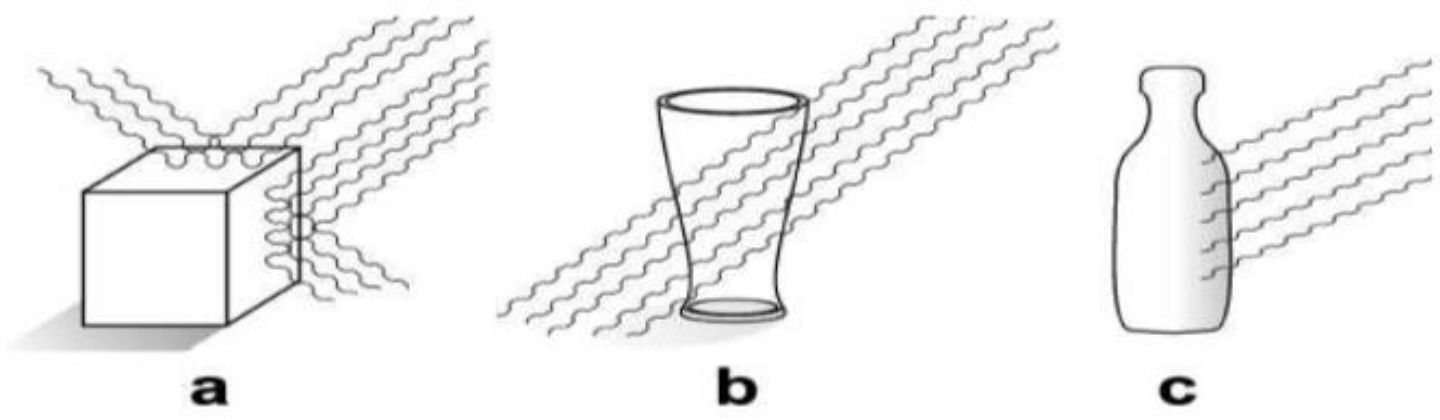

Figure 2.3: Subfigure a: electrical conducting material, subfigure b: insulating material, subfigure c: dielectric material ${ }^{17}$.

\subsubsection{Permittivity}

The capacitance encountered in the formation of an electric field of a medium is denoted as the absolute permittivity. This can be expressed as the amount of charge needed to generate one unit of electric flux in the medium being studied. Permittivity in essence is a materials ability to store an electric field in the polarization of the medium ${ }^{19}$. A material's dielectric medium usually is expressed as the relative permittivity of the material, this term is commonly called the dielectric constant in literature ${ }^{2,23}$. It can be expressed as kappa $\kappa$ which is the ratio of the absolute permittivity to the electric constant. Dielectric constant is not typically constant, it varies with position in the medium, the frequency of the field applied, humidity, temperature, and other parameters. In a nonlinear medium the dielectric constant can vary with the strength of the applied electric field ${ }^{19}$.

$$
\kappa=\varepsilon_{r}=\frac{\varepsilon}{\varepsilon_{0}}
$$

The dielectric constant is directly proportional to the electric susceptibility $\chi$, which is a measurement of how easily a dielectric polarizes in response to an electric field. The relation of these terms is given in equations 4 and 5 .

$$
\begin{gathered}
\chi=\kappa-1 \\
\varepsilon=\varepsilon_{r} \varepsilon_{0}=(1+\chi) \varepsilon_{0}
\end{gathered}
$$

The two main points of wave-matter interactions can be expressed by the two components of the dielectric constant.

$$
\varepsilon=\varepsilon^{\prime}-j \varepsilon^{\prime \prime}=\varepsilon_{0} \varepsilon_{r}^{\prime}-j \varepsilon_{0} \varepsilon_{r}^{\prime \prime}
$$

Where $\varepsilon^{\prime}, \varepsilon^{\prime \prime}, \varepsilon_{r}^{\prime}$, and $\varepsilon_{r}^{\prime \prime}$ are the real and imaginary parts of the complex dielectric permittivity and the real and imaginary parts of the relative complex dielectric permittivity. The ability of a material to store electromagnetic energy is expressed as the real part and the thermal 
conversion potential is proportional to the imaginary part $^{24}$. In Figure 2.4 the effects of different process on both parts of the permittivity are shown.

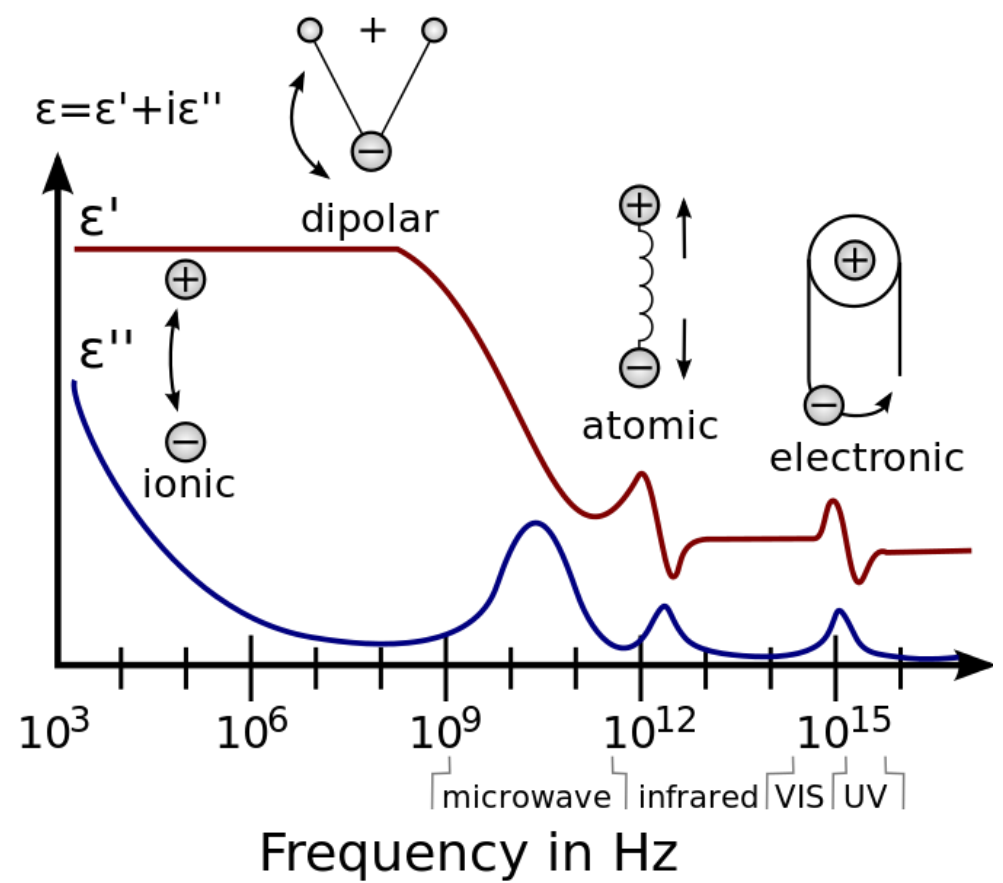

Figure 2.4: Dielectric permittivity spectrum over a wide range of frequencies.

Various processes are labeled on the image: ionic and dipolar relaxation, atomic and electronic resonances at higher energies ${ }^{67}$.

The electric loss of a material can be expressed in terms of the real and imaginary parts of the relative complex dielectric permittivity. This term is commonly referred to in literature as the loss tangent of a material. The most important aspect of the loss tangent is that it is proportional to a material's ability to absorb heat ${ }^{19,24,25}$.

\subsubsection{Permeability}

$$
\tan \delta_{e}=\frac{\varepsilon^{\prime \prime}}{\varepsilon^{\prime}}
$$

In electromagnetism the degree of magnetization of a material in response to a magnetic field is termed permeability ${ }^{24,25}$. The permeability is a measurement of the ability of a material to support the formation of a magnetic field within itself ${ }^{19}$. Permeability is directly related to the magnetic field $\vec{B}$. The relation is shown in Equation 8, where $\vec{H}$ is the auxiliary magnetic field. The auxiliary magnetic field influences dipole migration and magnetic dipole reorientation in a medium resulting in different magnetic fields ${ }^{19}$.

$$
\vec{B}=\mu \vec{H}
$$

The permeability is termed $\mu$, it is a second rank tensor for an isotropic medium and a scalar for an isotropic medium ${ }^{26}$. Like permittivity, permeability is not typically constant, it varies 
with position in the medium, the frequency of the field applied, humidity, temperature, and other parameters. In a nonlinear medium the dielectric constant can vary with the strength of the applied magnetic field ${ }^{27,28,29}$.

Like permittivity, there is a relative permeability that is merely the ratio of the permeability of a specific medium to the permeability of a free space. The relative permeability is directly related to the magnetic susceptibility ${ }^{19}$.

$$
\begin{gathered}
\mu_{r}=\frac{\mu}{\mu_{0}} \\
\chi_{m}=\mu_{r}-1
\end{gathered}
$$

The relative permeability has both a real and imaginary portion just like that of the dielectric constant. Where the real part is the magnetic permeability and the imaginary part is the magnetic loss. The ratio of which is a measure of how much power is lost in a material versus how much is stored ${ }^{19}$. The losses of a material are induced by the domain walls and from the electron spin resonance ${ }^{12,19,30}$. The material can be placed at the magnetic field maxima in order to allow for maximum absorption of microwave energy ${ }^{12,19,30}$.

$$
\begin{gathered}
\mu=\mu^{\prime}-j \mu^{\prime \prime}=\mu_{0} \mu_{r}^{\prime}-j \mu_{0} \mu_{r}^{\prime \prime} \\
\tan \delta_{m}=\frac{\mu^{\prime \prime}}{\mu^{\prime}}
\end{gathered}
$$

\subsubsection{Microscopic, Local and Macroscopic Fields}

When a semi-infinite medium has an electromagnetic field applied the material will show effects from the particle back-reaction field and the applied field ${ }^{19}$. This results in charges and spins inside the medium which react with the local fields but not directly with the applied field. In the case of a dielectric material, the surface-charge dipole-depolarization fields that oppose the applied field will change the macroscopic and local fields in the material when interacting with an electromagnetic field ${ }^{19}$. This relationship grows more complex as consideration is made for the time-dependent high-frequency fields. In this circumstance the dipole orientations and thus the electromagnetic fields can be affected by a number of factors, including depolarization, demagnetization, thermal expansion, exchange, and anisotropy interactions ${ }^{12,19,30}$.

When modeling the relationships between the applied, macroscopic, local, and microscopic fields a special attention must be payed to where the fields originate from and their interactions with one another. External charges generate the applied fields, while the macroscopic fields are merely the averaged quantities in the medium. The macroscopic fields can be implicitly defined through the constitutive relationships with boundary conditions.

\subsubsection{Local Electromagnetic Fields in Materials}

The effective local field is commonly defined as the Lorentz field in literature, where the Lorentz field is defined as the field in a cavity that is varved out of a material around a specific 
site. The local field for a Lorentz spherical cavity can be defined as the sum of the applied $\left(E_{a}\right)$, depolarization $\left(E_{\text {dep }}\right)$, Lorentz $\left(E_{\text {Lorentz }}\right)$, and atomic fields $\left(E_{\text {atom }}\right)$ as shown in Equation $13^{16,19,30}$.

$$
E_{l}=E_{a}+E_{\text {dep }}+E_{\text {Lorentz }}+E_{\text {atom }}
$$

Where for a cubic lattice in the sphere the applied field can be related to the macroscopic field and polarization by Equation $14^{16}$.

$$
E_{l}=E+\frac{1}{3 \varepsilon_{0}} P
$$

This polarization field (P) for a molecule is expressed as a function of the local field (E), which can in turn be expressed as a function of the macroscopic field. These relationships can be seen in equations 15 and $16^{16}$.

$$
\begin{aligned}
& p \approx \alpha E_{l} \\
& E_{l}=\beta E
\end{aligned}
$$

The relationship is between the fields is difficult to calculate, this is due to the local field's relationship to the macroscopic field, the polarizabilities, permittivity, and permeability absorb parts of the local field.

\subsection{Measurement Models for Material Properties}

\subsubsection{Transmission/Reflection measurement techniques for lossy materials}

The characterization of microwave material interactions can be defined as a mathematical model, where the waves that are reflected and transmitted through the material at a certain frequency are measured. In a transmission/reflection (TR) measurement, a material is placed into a waveguide or coaxial line and subjected to a microwave with a known frequency ${ }^{26}$. The material reflects part of that wave while allowing for some of it to pass through. The study of this effect revels the specimen's dielectric properties. The reflection and transmission data are known as scattering data. The scattering data must be solved using the electromagnetic boundary-value problem in order to determine the materials properties ${ }^{26}$.

\subsubsection{Scattering Parameters}

Scattering parameters (S-parameters) are a type of small-signal AC commonly used to characterize RF components. S-parameters establish small-signal characteristics of a device at a specific bias and temperature ${ }^{31}$. They are measured by making the measuring device impeded between a 50-ohm load and a source, drastically reducing the chance of oscillations to occur. Sparameters have the distinct advantage of not varying in magnitude at points along a lossless transmission line because they are traveling waves not terminal voltages ${ }^{31}$. A signal wave for a two-port electrical-element is represented in Figure 2.5, where $\mathrm{a}_{1}$ is the wave into port $1, \mathrm{a}_{2}$ is the wave into port $2, b_{1}$ is the wave out of port 1 , and $b_{2}$ is the wave out of port 2 . 


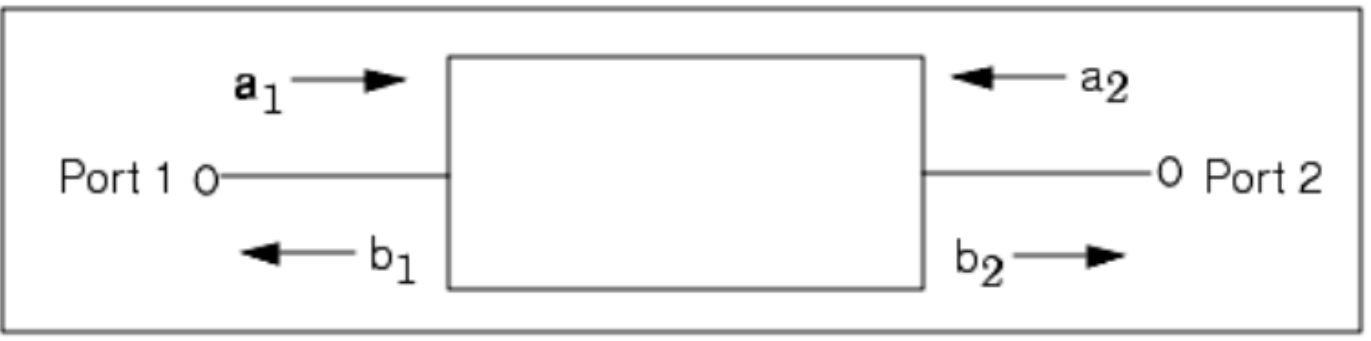

Figure 2.5: Single wave in a two-port electrical-element. Simple representation of a standard 2 port measurement for S-parameters ${ }^{32}$.

A conventual element S-parameters for microwaves can be defined as in equations 17 and $18^{18}$. Where $\mathrm{s}_{11}$ is the port- 1 reflection coefficient, $\mathrm{s}_{22}$ is the port- 2 reflection coefficient, $\mathrm{s}_{21}$ is the forward transmission coefficient, and $\mathrm{s}_{12}$ is the reverse transmission coefficient. For a 50 ohm system with the two-port setup, each port is terminated at $50 \mathrm{ohms}$ and the $\mathrm{s}_{21}$ parameter represents the voltage gain of the element from port 1 to port $2^{32}$. S-parameters are commonly displayed as magnitude plus phase of the wave being measured or as a real plus imaginary number converted from the magnitude and phase.

$$
\begin{aligned}
& b_{1}=a_{1} s_{11}+a_{2} a_{12} \\
& b_{2}=a_{1} s_{21}+a_{2} a_{22}
\end{aligned}
$$

\subsection{Instrumentation}

There are several methods used in literature to measure the above-mentioned material properties each with different strengths and weaknesses. No one technique can characterize every material for every frequency leading to a need for a plethora of techniques based on several key factors. The selection of a measuring techniques depends on several significant factors such as, frequency, accuracy, temperature, material nature, sample size and or thickness, containing or noncontacting, destructive or non-destructive and cost. Most dielectric property measurement techniques can be broken into two categories; resonant and non-resonant ${ }^{33,34}$. Resonant methods characterize materials at discreet frequency points where the dielectric material is used as a resonant element ${ }^{33}$. The drawbacks to this method are that the sample must be a low loss material. The resonant method technique also known as perturbation method requires samples to be placed into a resonant cavity ${ }^{33}$. The resonant cavity causes perturbations that result in resonant frequency shifts. This form of the resonant method is used for low to moderate loss samples ${ }^{33,34}$.

The non-resonant method is used to measure frequencies over a broad range. This technique is the more prevalent one in literature as it utilized the transmission and reflection coefficients discussed in section 2.3.2 $2^{33}$. Some of the most common non-resonant techniques in literature are the coaxial airline method, waveguide method, free space method, and coaxial probe method $^{33,34}$.

\subsubsection{Coaxial Line}

The coaxial airline is part of the transmission line method, utilizing the measurement of a reflected signal and transmitted signal. A coaxial transmission line is a cylindrical test cell with a 
center conductor running concentric, cut to the exact length as the test cell, a schematic is shown in Figure 2.7. The coaxial line is characterized by the material filling the entire cross section with no air gaps existing at the walls ${ }^{33}$. The coaxial line technique can measure magnetic materials, it is however limited at its lower frequencies based on the sample lengths. The coaxial airline technique can cover a broad frequency range and is best for lossy machinable solids ${ }^{31}$.

The coaxial airline method is one of the transmission line methods commonly used in literature and has their associated advantages and disadvantages. These advantages include the ability to cover a wide frequency range from $50 \mathrm{MHz}$ to more than $100 \mathrm{GHz}$ as well as to measure anisotropic materials. Coaxial airlines are used to measure solid and powdery materials with low to medium $\operatorname{loss}^{33,35}$. It is one of the most accurate measuring techniques available for the testing of material properties. This is because the bandwidth of the coaxial airline is smaller than that of some of the other techniques discussed in this study. The presence of a center conductor in the coaxial airline method avoids the creation of any higher order modes that would cause increased error $^{33,35}$.

The disadvantages of the transmission/reflection line methods include the air-gap effects and the difficulties of discontinuities associated with samples that are multiples of one-half wavelengths ${ }^{33,35}<$ sup $>35</$ sup $>$. The presence of the center conductor makes the creation of testing samples slightly more difficult than other methods as well as reduces the amount of power that the testing cell can handle 33,35 .

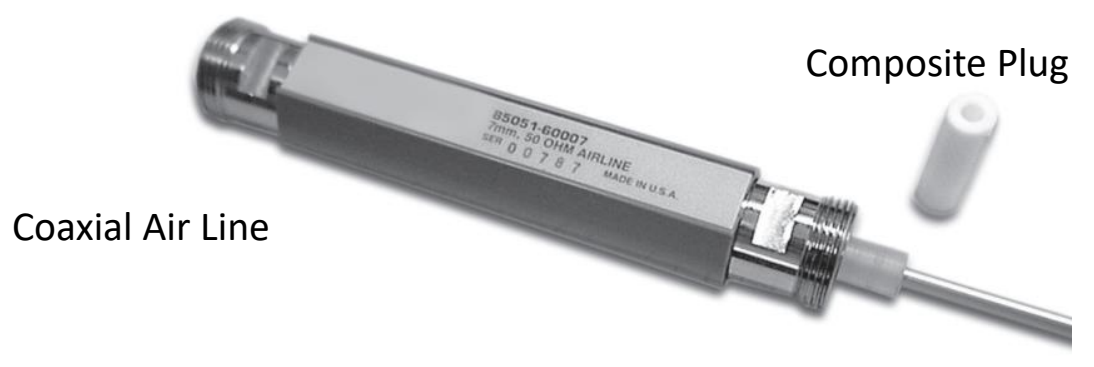

Figure 2.6 Keysight Network high precision coaxial airline. Used to measure the scattering parameters and calculate the associated material properties. Example of a testable material is shown as the composite ${ }^{36}$.

\subsubsection{Waveguide}

The waveguide measurement technique can take several different shapes but is typically a rectangular cross section, a schematic is shown in Figure 2.8. Like the coaxial line, the waveguide method requires the sample to fill the entire cross section and no airgaps to exist at the walls ${ }^{33}$. The waveguide technique can also measure magnetic materials with limitation at lower 
frequencies. The waveguide method can measure anisotropic materials while the coaxial method cannot. High precision machining of samples is also required for the waveguide ${ }^{31}$.

When using the waveguide method to measure material properties it shares many of the same advantages and drawbacks as the coaxial method as they are both transmission line measurements. The waveguide method is used to measure samples with medium to high loss ${ }^{33,35}$. Waveguides have easy to manufacture shapes making sample preparation comparatively simple. Since the waveguide is filled with only air and the sample, no power is lost through radiation with dielectric loss being negligible. At high power the waveguide technique is able to give accurate measurements because of the fact that air has a breakdown voltage of $30 \mathrm{KV} / \mathrm{cm}^{33,35}$.

The disadvantages associated with all transmission/reflection line methods have to do with the disassociations when the sample is a multiple of one have wavelength and the effects of airgaps on the sample. For waveguides in particular, the outer wall has to be thick making it quite expensive and susceptible to power loss through heating. The lack of an inner conductor limits the measurable bandwidth of the waveguide and make it vulnerable to the generation of higher order modes within the waveguide ${ }^{33,35}$.

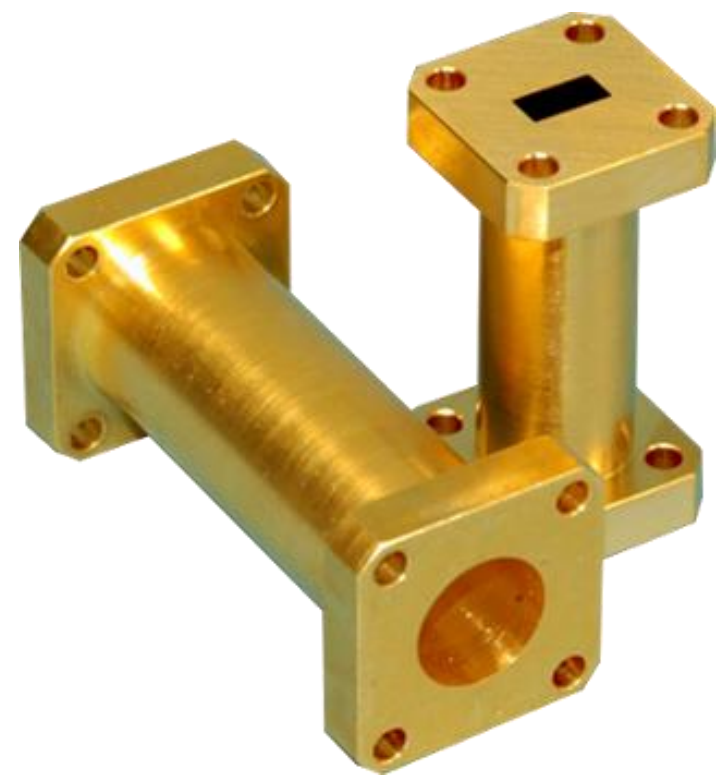

Figure 2.7: Waveguides for use in vector network analyzer, shown are both a circular and rectangular waveguide ${ }^{68}$.

\subsubsection{Free Space}

Free-space measurement techniques use antennas that focus microwave energy at or through a slab of material without the need for a test setup. This method assumes that the material being measured is large, flat, and is a parallel faced sample. The sample is also assumed to be homogenous during testing. This free-space measurement is non-contacting as well as nondestructive. Free-space measurements are done at high frequencies and can sustain operations at high temperatures. An illustration of the free space method is shown in Figure 2.9. The free space 
method can have their antenna polarization varied for anisotropic materials and can measure magnetic materials ${ }^{31,33}$.

When using the free space method to measure material properties there are several advantages of using this technique over the others discussed in this study. The free space method covers a wide range of frequencies while requiring no contact. Free space sample preparation is very easy as it is best for large flat solid materials and can be tested at high temperatures ${ }^{33,35}$. It has relatively moderate accuracy for high and low loss materials. Some of the problems associated with this method are the diffraction problem from the material edges, along with a low end threshold based on the practical sample size $\mathrm{e}^{33,35}$.

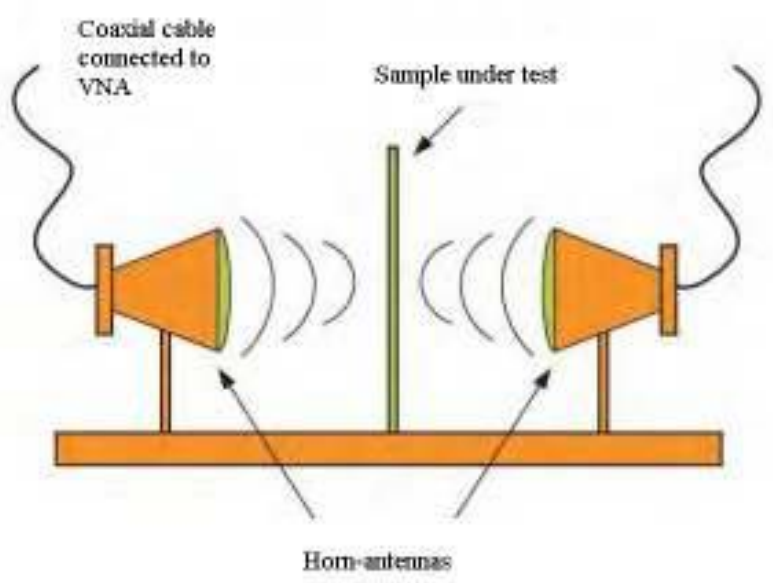

Figure 2.8: Illustration of the Free space method during testing of material properties ${ }^{31}$.

\subsubsection{Coaxial Probe Method}

Open ended coaxial probe method measures a material by immersing the probe into a liquid or touching it to the surface of a solid (or powder). The probes electromagnetic field changes as it comes into contact with the material allowing for a measurement of the reflected signal $\left(\mathrm{S}_{11}\right)$ and related to the dielectric constant of the material. The coaxial probe method is rated to test materials in the range of -40 to $+200{ }^{\circ} \mathrm{C}$, leaving it often termed as a high temperature probe. The sample being measured must be appear infinite to the probe, meaning there must be enough of the sample so that the thickness allows accurate calculations ${ }^{31}$. Figure 2.10 shows a Keysight coaxial probe that comes as standard with the $\mathrm{VNA}^{36}$.

The coaxial probe method has several distinct advantages it covers broadband frequencies, it can measure isotropic and homogenous materials, sample preparation is easy and fast, it is nondestructive and has very high accuracy for high-loss materials. This method is best when used to measure semi-solids or liquids rather than large solid materials ${ }^{33,35}$. Some of the drawbacks for this method include the above discussed airgaps that can cause errors in the measuring of material properties. This technique also required repetitive calibrations in order to produce repeatable 
results $^{33,35}$. Typically, the coaxial probe method requires a large amount of sample material to achieve material measurements.

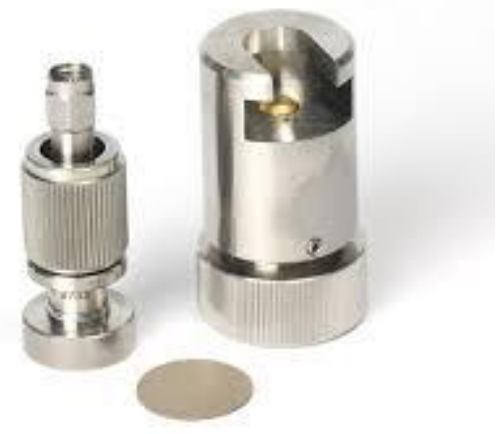

Figure 2.9: Standard Keysight High Temperature Coaxial Probe, used to collect scattering parameters from a material so a VNA can calculate the material properties ${ }^{36}$.

\subsubsection{Network Analyzers}

Network analyzers are the preferred method for the collection of data on electromagnetic wave, material interactions. Network analyzers work by measuring the scattering parameters in order to characterize a material. Vector network analyzers (VNA) measure both amplitude and phase, allowing for more detailed information to be gathered about the material being measured, an example is shown in Figure 2.10. Network analyzers are subject to various sources of error such as, Nonlinearity of mixers, gain and phase drifts in amplifiers, noise introduced by the analog to digital converter, imperfect tracking in dual channel systems, imperfect matching at connectors and imperfect calibration standards ${ }^{26,36}$. 


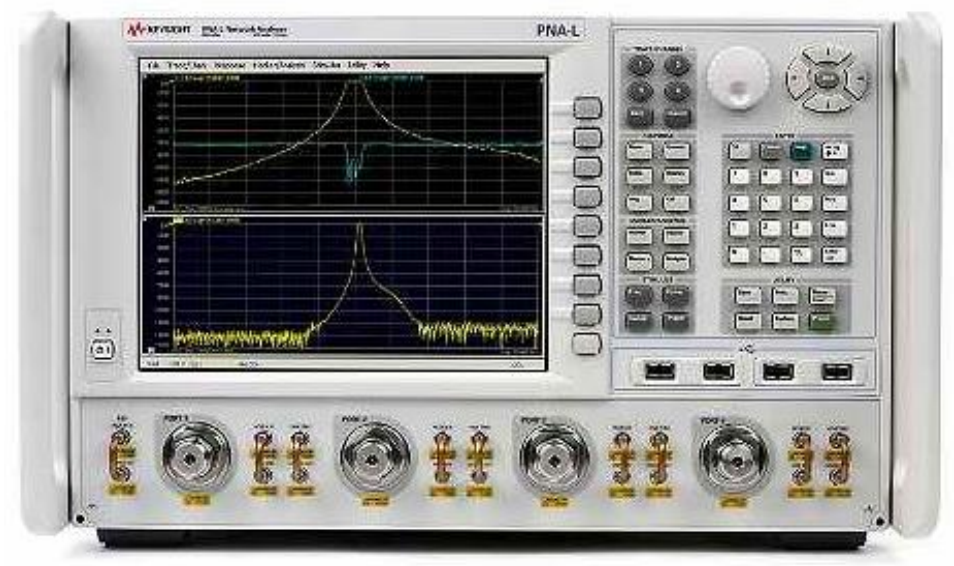

Figure 2.10: Keysight Network Analyzer part of the vector network analyzer family of machinery. Used primarily to measure the scattering parameters and calculate the associated material properties. VNA's are used for many of the different techniques mentioned in the following sections ${ }^{36}$.

\subsection{Error}

\subsubsection{Random Uncertainties and Error}

In transmission/reflection measurement techniques there are several different types of error, one of them being random uncertainties of the calibration and from the specimen itself. The three main types of random uncertainties and error sources typically related to transmission/reflection measurements are errors in measuring the magnitude and phase of the scattering parameters, error in specimen length and error in reference plane positions ${ }^{26}$. To counteract this problem a differential uncertainty analysis can be applied to both $\mathrm{s}_{11}$ and $\mathrm{s}_{21}$ separately. For both $\mathrm{s}_{11}$ and $\mathrm{s}_{21}$ the dominate uncertainty is the phase, with longer specimens having less uncertainty. It has been found in literature that at higher frequencies S-parameters have larger uncertainties in phase ${ }^{26}$.

\subsubsection{Systematic Uncertainties}

The other type of error associated with transmission/reflection measurements is systematic uncertainties. These uncertainties can be broken down into several main types, gaps between the specimen and specimen holder and specimen holder dimensional variations and line losses and connector mismatch $^{26}$. There are standard equations in literature that are made to handle the first type of uncertainties for gaps around the specimen ${ }^{37,38}$. Along with airgaps other systematic uncertainties include short-circuit and waveguide wall imperfections and $\operatorname{losses}^{26}$. Waveguide losses can be corrected with by taking a measurement of an empty waveguide and calculating the appropriate correction factor or attenuation coefficient. For airgaps additional measurements using a resonator of the same material in the frequency band being measured will determine the required gap in the correction formula.

\subsubsection{Corrections to Data}

With the many possible errors associated with dielectric measurement testing and the difficulty of data collection, corrections must be made once a measurement has been obtained. The 
corrections must account for the systematic uncertainties and if possible the random uncertainties and error. Known uncertainties associated with transmission/reflection measurements are airgaps around the sample, wall imperfections, and losses. Airgap corrections are most important when considering the coaxial method of testing with particular emphasis on the center conductor ${ }^{26}$. For both coaxial and waveguide method, airgap correction are particularly important in whichever region has the strongest electromagnetic field ${ }^{37,38}$. Both waveguides and coaxial lines at ambient temperature will experience some power loss because they are not perfectly conducting. The different propagation modes will be attenuated to some degree because of this power loss ${ }^{26}$.

\subsection{Permeability and Permittivity Calculations}

\subsubsection{Nicolson-Ross-Weir}

The most widely used method for calculating permittivity and permeability from Sparameters is the Nicolson, Ross, Weir method (NRW) $)^{39,29}$. This method is applicable over the range of $100 \mathrm{MHz}$ to $18 \mathrm{GHz}$ when using a computer-controlled network analyzer. However it is subject to singularities when the specimen length is a multiple of one-half wavelength in the materia ${ }^{26}$. This is especially prevalent for low-loss materials as it is impossible to measure the phase of $S_{11}$ accurately. NRW takes measurements in the frequency domain rather than the time domain ${ }^{24,25}$ which avoids the need of using a Fourier transform to calculate permittivity and permeability. The NRW method works for both waveguides and coaxial lines using discrete frequencies in less than $20 \mathrm{kHz}$ steps ${ }^{29}$.

\subsubsection{Nicolson-Ross-Weir Polynomial Method}

The NRW polynomial method is an iterative technique to fit material properties to a polynomial. This method increments the method of the polynomial until the difference between Sparameters calculated from the polynomial and the measured S-parameters are less than the expected system performance. This method can also be done using a user specified maximum order for the polynomial ${ }^{23,40}$.

\subsubsection{NIST Iterative Solution}

For a general iterative solution if the material being tested has a known length and reference plane it is possible to use an iterative solution to solve for the permittivity and permeability. Using various linear combinations of the scattering equations and solving them iteratively will result in a stable solution for permetivity ${ }^{26}$. This iterative method however can be unstable when solving for both permittivity and permeability, with integrals of one-half wavelength causing the instability due to the TEM mode resonance. The NIST iterative solution is only suitable for permittivity calculations as it assumes the permeability is equal to one. A solution is obtained using a NewtonRaphson's root finding method ${ }^{35}$. The common inaccuracy peaks associated with the NRW method are avoid when using the NIST iterative method, making it suitable for long samples and low loss materials ${ }^{35}$. However, a disadvantage of this method is the need for a good initial guess.

\subsubsection{NIST Non-Iterative}

The NIST non-iterative method like the iterative method is only applicable for permittivity calculations as it also assumes the permeability of one. The non-iterative method is similar to the NRW method but with a different formulation of equation based on the assumption of permeability of one. Unlike NRW this method is stable over the whole range of frequencies for any sample 
length. An advantage the non-iterative method has over the iterative one is the lack of an initial guess with both of them providing high accuracy. The non-iterative method can also be solved very when compared to some of the other methods ${ }^{35}$.

\subsubsection{Short Circuit Line}

The short circuit line (SCL) method is used in coaxial and waveguide measurements and features only one port. Like the NIST methods the SCL method is applicable for permittivity only and uses the same Newton-Raphson numerical approach ${ }^{35}$. This method like the iterative one requires a good initial guess in-order to avoid convergence to any local optima ${ }^{41}$. The SCL method is best for broadband measurements with ling samples and low loss materials. The distance from the sample to the short-circuit termination must be known to a high degree of accuracy in order to obtain usable results. To obtain the permittivity a strong electric field is preferable for accurate results ${ }^{41}$.

\subsection{Microwave Heating}

The use of microwave heating for chemical applications such as chemical synthesis, catalyst, analytical chemistry, materials, polymers, drug discovery and biochemistry has become a major foundation of research ${ }^{21,20,42}$. The use of microwave energy to treat materials such as vermiculite is a long-standing practice. However, there persist a lack of understand about the dielectric behavior of this material in the microwave regime. An understanding of vermiculites dielectric behavior would allow microwave heating to be used for electrical separation technology $y^{20}$. This technology can be used in the separation of vermiculite ores from gangue materials during mining ${ }^{20}$. Microwave heating has been used extensively in chemical processing to the effect of controlling the shape of powder particles ${ }^{21}$. This is accomplished by controlling the energy delivered to the processes and rapid production of the particles. The dielectric constant controls the anomalous behavior of the powder particles at a microscopic scale, resulting in different thermal distributions. The key controlling factor that allows the process of microwave heating to be achieved is the dielectric constant of the material being processed.

\subsection{Powdery Materials}

Powdery materials are good for many applications because of several important factors, the dominate one being there incredibly high surface area. Fine powders have an exposed surface area of over a million times greater than that of undivided material ${ }^{43}$. Powdery materials physical behavior is governed interparticle forces, in particular the gas-particle interaction, geometry of particle positions and geometry of particle contacts when in ambient gases ${ }^{43,44}$. When the particles are small and dry the interparticle attractive forces are much higher than the single particle weights leading to aggregation. The strong attractive forces and strong gas interactions experienced by powdery material are what sets it apart from the behavior of large solids ${ }^{44}$.

The coupling of microwaves with powdery catalyst materials is a very active area of research with many promising results ${ }^{15}$. Heterogenous catalyzed reactions provide desirable reaction fields. If selected heating a unique property of microwaves is applied to certain processes it can provide incredibly unique results. if a nonpolar sample or gas are involved in a microwave catalyzed chemical process then this would result in only the catalyst being heated. The 
understanding of heterogenous powdery catalyst can greatly increase the possible reactions used in a multitude of fields.

\subsection{Specimen Preparations for Powdery Material}

\subsubsection{Sintering}

Powdery sample preparation for many different procedures requiring a process called sintering to form the desired shape and material characteristics. Sintering involves heating a powder at high temperatures any typically also involves pressing a powder into a desired temperature. Sintering effects many different physical attributes of powders including porosity and grain size resulting in changes to hardness, yield stress, compressive strength, and wear resistance ${ }^{45}$. For most powders as sintering temperature increases grain size increases while porosity is reduced ${ }^{45}$, this makes finding a balance between them to maximize mechanical behavior an important factor in powder processing. It has also been found in literature that sintering has a direct effect on density and lattice structure ${ }^{46}$. These effects can have repercussions on the dielectric constant and other parameters affecting them in unknown and uncontrollable ways. Sandi et al. shows a direct correlation between sintering temperature and dielectric constant ${ }^{47}$. Sintering can help in many processes to form the desired shape, but sintering can also have unknown and unpredictable consequences on internal material parameters. As figure 2.11 shows sintering has a drastic effect on particle morphology ${ }^{48}$, this is especially true for multi-phase powders like catalyst. These changes in morphology as a result of sintering would render most catalyst useless.

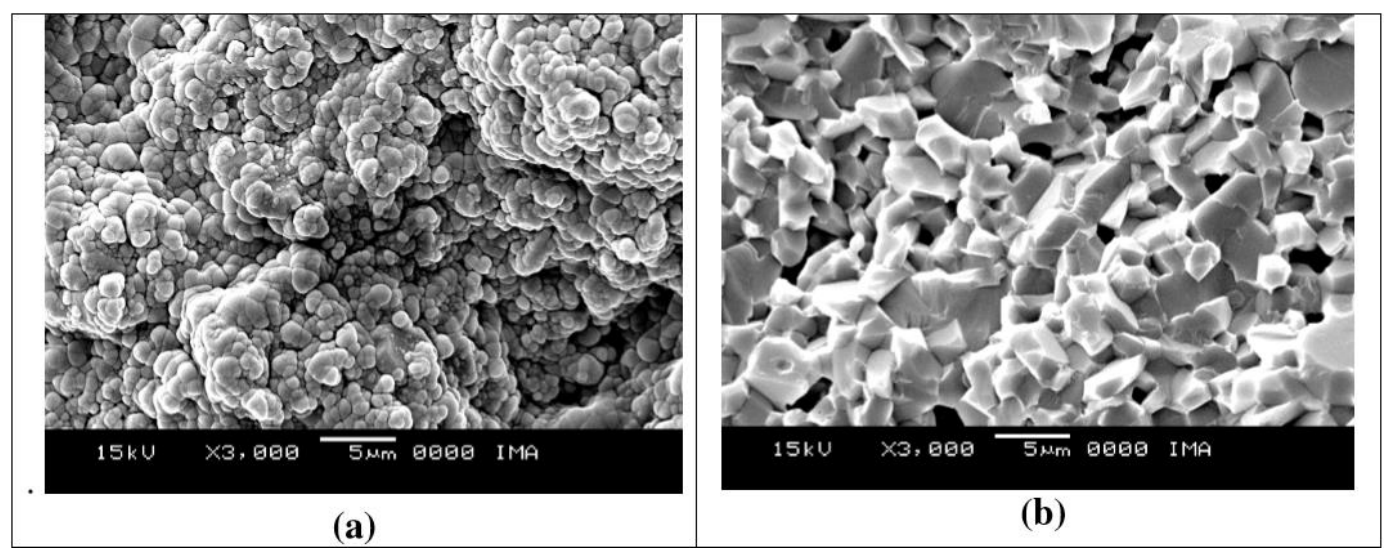

Figure 2.11: (a) Alumina pellet with individual grains, (b) well sintered pellet with defined cleavage plans at $1580^{\circ} \mathrm{C}^{48}$.

\subsubsection{Pressing}

Pressing uses pressure to form powder into the desired shape, either using hot or cold pressing $^{49}$. Hot pressing involves packing a powder into the desired shape at an elevated temperature. This technique is usually coupled with sintering to achieve the desired shape. Cold pressing is accomplished by applying pressure on a loose column of powders in a closed die to form a compact powder ${ }^{50}$. It can be further classified into two more types of pressing, axial pressing or isostatic pressing. The effects of pressure on powders is highly dependent on their particle size, shape, composition, and size distribution ${ }^{49}$. Pressing powders has several drawbacks 
with the uneven distribution of density causing the most problems in industrial and scientific applications. Some of the other common problems with powder pressing are punch and die fractures, slip cracks and cleavage fractures ${ }^{49}, 50$. Figure 2.12 shows the effect of particle size in powder pressing and defines well the uneven distribution of density always present in this methodology $\mathrm{y}^{51}$.

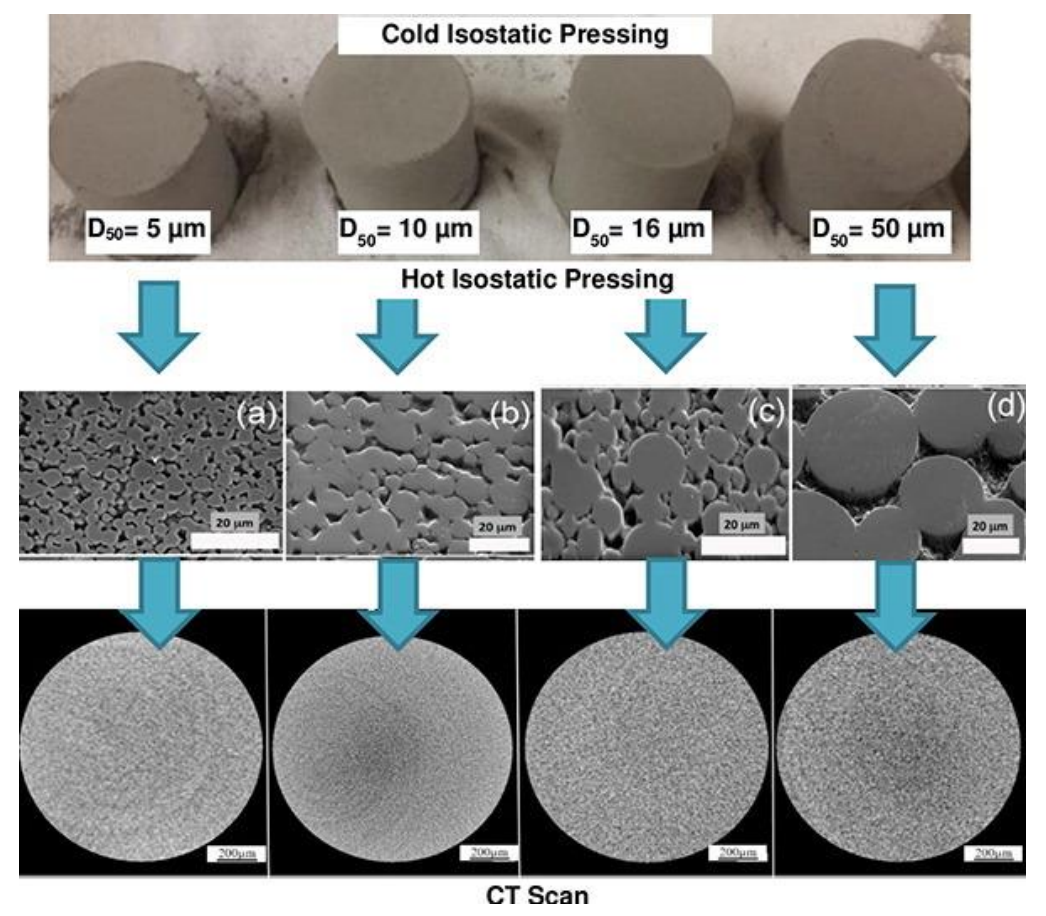

Figure 2.12: Effects of particle size on both hot isostatic pressing and cold isostatic pressing ${ }^{51}$.

\subsubsection{Paraffin Matrix}

Paraffin wax is widely used in literature to set powders into a matrix for scientific testing, it is a cheap and well characterized material. It comes in several different forms with fully refined solid paraffin existing at room temperature with a melting point of around $58{ }^{\circ} \mathrm{C}{ }^{52,53}$. This low melting point and well understood characteristics make it an obvious choice for room temperature dielectric testing. The wax can be melted and combined with powders at different volume loadings using mechanical agitation. The two-phase mixture can be cast into whatever form is desired for testing. This methodology has been around since the 1950's and requires the usage of two-phase homogenous mixture equations in order to calculate the dielectric constant of the powder ${ }^{91,54}$. This methodology permits for control over many of the variables that are lacking in the other methods. Things such as the elimination of air within the sample as well as control over the exact amount of the two-phases being used. One of the big advantages of using a paraffin matrix is that it is accomplished at below $75^{\circ} \mathrm{C}$ thereby avoiding undesirable sintering effects. This ensures it has no limits in the type of powder that can be measured. The different mixture equations associated with this method along with their benefits and drawbacks will be talked about in section 2.8. Using this 
methodology allows for the reduction of unknown variables resulting in more accurate test results then the other methods presented in this paper.

\subsection{Mixture equations for Two Phase Homogenous Composites}

The process of determining the dielectric constant is a complex process with no exact solution existing. There are several equations in literature that attempt to take the dielectric constant of two different materials and predict their combined dielectric constant. These equations are of use in the paraffin composite methodology as they can be set to solve for the dielectric constant of the constitutive material with the composites dielectric constant being experimentally tested. These equations are almost exclusively based on only four variables, the dielectric constant of the powder and its volume loading, the dielectric constant of the inclusion matrix and its volume loading and the dielectric constant of the two-phase composite. The EMT mixture equation discussed in section 2.8.8 has an additional variable of a shape factor constant. The equations all aim to predict the behavior of the dipole-dipole interactions between the different phases of the materials, with varying degrees of success ${ }^{19}$. The difficulty in predicting the dielectric constant of a two-phase homogenous composite lies in the fact that no exact solution existing for the electrostatic problem in a random heterogenous geometry ${ }^{1}$. This study focuses on measuring the dielectric constant of the two-phase mixture and back-calculating the dielectric constant of powders dielectric constant.

\subsubsection{Parallel Mixing Equation}

One of the standard equations for predicting the dielectric constant of a two-phase homogenous composite is the parallel mixing equation. Equation 19 shows the parallel mixing equation also sometimes called the linear law, it is part of a family of predictive equations called the power law family. This formula is abdicable if the theoretical configuration of the mixture is formed of plates or other types of inclusion where no depolarization is induced ${ }^{55}$. This mixture equation is made to mimic that of a parallel plate capacitor. The following sections use the same nomenclature where $\varepsilon_{m i x}, \varepsilon_{m}$, and $\varepsilon_{p}$ stand for the dielectric constant of the mixture composite, matrix material (paraffin), and powdery material respectively. $V_{p}$ is the volume loading of the powder and $V_{m}$ is the volume loading of the paraffin ${ }^{1}$.

$$
\varepsilon_{m i x}=V_{p} \varepsilon_{p}+V_{m} \varepsilon_{m}
$$

\subsubsection{Series Mixing Equation}

Another equation for predicting the dielectric constant of a composite is the series mixing equation. Equation 20 is setup so that the dielectric constant of the components behave like that of a series capacitor ${ }^{54}$.

$$
\left(\varepsilon_{m i x}\right)^{-1}=V_{p}\left(\varepsilon_{p}\right)^{-1}+V_{m}\left(\varepsilon_{m i x}\right)^{-1}
$$




\subsubsection{Logarithmic Mixing Equation}

The Logarithmic mixture equation can be shown to be derive from the famous MaxwellGarnet equations ${ }^{56}$. It is another empirical equation that aims to predict the behavior of two intermixed dielectric constants. The formula was obtained by further assuming a random distribution of shapes and orientations for each component enabling the charge density at any position to be replaced by the mean charge density of the mixture ${ }^{56}$.

$$
\ln \left(\varepsilon_{m i x}\right)=V_{p} \ln \left(\varepsilon_{p}\right)+V_{m} \ln \left(\varepsilon_{m}\right)
$$

\subsubsection{Lichtenecker Mixing Equation}

The Lichtenecker mixing equation is another of the power-law family of equations. This equation averages the logarithms of the permittivity's in order to mathematically predict the dielectric constant of the mixture ${ }^{55}$.

$$
\varepsilon_{\text {mix }}=\varepsilon_{p}^{V_{p}} * \varepsilon_{m}^{V_{m}}
$$

\subsubsection{Looyenga Mixing Equation}

The Looyenga mixture equation is one of the differential mixing models available in literate. It attempts to mathematically predict the internal microstructure interaction between dipoles. These equations are derived based on differential analysis rather than on first principle calculations. This equation is considered to be similar to the Bruggeman family of equations because of the symmetry between matrix and inclusion ${ }^{1}$.

$$
\varepsilon_{m i x}{ }^{1 / 3}=V_{m} \varepsilon_{m}{ }^{1 / 3}+V_{p} \varepsilon_{p}{ }^{1 / 3}
$$

\subsubsection{Birchak Mixing Equation}

The Birchak mixing equation is also part of the power-law family of equations were the permittivity is averaged by volume weights. For this formula the square roots of each components permittivity add up to the square root of the mixtures permittivity. This mathematical model also can be used to state that the refractive index of a mixture's nonmagnetic gases is the volume average of the indices of each component a useful tool in optical physics ${ }^{1}$.

$$
\varepsilon_{m i x}{ }^{1 / 2}=V_{m} \varepsilon_{m}{ }^{1 / 2}+V_{p} \varepsilon_{p}{ }^{1 / 2}
$$

\subsubsection{Poon-Shin Mixing Equation}

The Poon-Shin Mixture equation is derived from the polarization of each inclusion related to the electric field along with the electrical displacement as a function of electrical field and polarization. The Poon-Shin model revolves around the idea of the displacement field as a function of a single particle's sum forces. Those forces are broken down to the medium and the polarization

of the particles that are embedded in the medium ${ }^{5754}$. This equation is desired for when the 
dielectric constant of the inclusion particle is greater than that of the matrix material, for our application this translates to $\varepsilon_{p}<\varepsilon_{m}{ }^{57}$.

$$
\varepsilon_{\text {mix }}=\varepsilon_{m}\left[1+\frac{V_{P}\left(\frac{\varepsilon_{p}}{\varepsilon_{m}}-1\right)}{V_{P}+\left(\frac{V_{m}}{3}\right)\left[\frac{\varepsilon_{p}}{\varepsilon_{m}} V_{m}+V_{p}+2\right]}\right]
$$

\subsubsection{Effective Medium Theory Mixing Equation}

The Effective Medium Theory mixing equation (EMT) is one of the Maxwell-Garnet family of equations. It is an algebraic formula were the mixtures dielectric constant is calculated as a function of the constituent permittivity's their volume fraction and a shape factor denoted $\mathrm{n}$. This model attempts to idealize the geometry and model the microstructure using simple forms ${ }^{30,54}$. The EMT mixing equation is designed to include first-order scattering effects of the inclusions. The contribution to the imaginary part of the effective permittivity is estimated by calculating the energy that the electric dipoles radiate ${ }^{30,55}$. Where $\mathrm{n}$ acts in the equation as a depolarization factor.

$$
\varepsilon_{\text {mix }}=\varepsilon_{m}\left[1+\frac{V_{p}\left(\varepsilon_{p}-\varepsilon_{m}\right)}{\varepsilon_{m}+n V_{p}\left(\varepsilon_{p}-\varepsilon_{m}\right)}\right]
$$

\subsubsection{Maxwell-Garnet Mixing Equation}

The Maxwell-Garnet Mixing equation is one of the oldest and most popular mixture equations for two-phase dielectric composites. The equation was derived based on ignoring the associated density fluctuations about a mean value and instead focusing on the dipole character of scattering ${ }^{1,58}$. This approach can be used to include the finite-size effects of scattering, with the main limitation being the lack of discrimination between two random media. This is especially true when the media has the same density of scattering but have different statistical distributions ${ }^{1,55,59,30}$. It is commonly claimed that weak particle interaction is a condition of the Maxwell-Garnet theory.

$$
\varepsilon_{m i x}=\varepsilon_{m}+3 * V_{p} \varepsilon_{m}\left(\frac{\varepsilon_{p}-\varepsilon_{m}}{\varepsilon_{p}+2 \varepsilon_{m}-V_{p}\left(\varepsilon_{p}-\varepsilon_{m}\right)}\right)
$$

\subsubsection{Jayasundere-Smith Mixing Equation}

The Jayasundere-Smith Mixing equation was derived in order to predict the dielectric constant of binary piezoelectric0-3 composites. Jaysundere and Smith derived their expression by modifying Kerner's expression. Which focused on the interactions of isolated dielectric spheres in a dielectric continuum. The system that this equation is design to handle is composed of piezoelectric sphere with a dielectric sphere dispersed in a constant medium. Where the spheres dielectric is always greater than that of the medium ${ }^{60}$. 


$$
\varepsilon_{\text {mix }}=\frac{\varepsilon_{m} V_{m}+\varepsilon_{p} V_{p}\left[\frac{3 \varepsilon_{m}}{\left(\varepsilon_{p}-2 \varepsilon_{m}\right)}\right]\left[1+\frac{3 V_{p}\left(\varepsilon_{p}-\varepsilon_{m}\right)}{\left(\varepsilon_{p}+2 \varepsilon_{m}\right)}\right]}{V_{m}+\frac{V_{p}\left(3 \varepsilon_{m}\right)}{\left(\varepsilon_{p}+2 \varepsilon_{m}\right)}\left[1+\frac{3 V_{p}\left(\varepsilon_{p}-\varepsilon_{m}\right)}{\left(\varepsilon_{p}+2 \varepsilon_{m}\right)}\right.}
$$

\subsection{Dielectric Constant Bounds}

\subsubsection{Wiener Bounds}

As previously discussed, different mixing models predict different permittivity values for the same mixture. These predictions are bound by a range of predictive values, with the loosest bounds being termed the Wiener bounds. The maximum bounds for the dielectric constant of the mixture is the same as Equation 18, where the equation mimics a parallel capacitor circuit ${ }^{1}$. In this equation the mixing formula is assuming an aligned ellipsoid with a depolarization factor of $0^{1}$. The minimum permittivity values for a mixture are given by the Equation 19 where the equation mimics a series capacitor circuit ${ }^{55}$. This equation also assumes aligned ellipsoids where the depolarization factor is 1 . These values hold true regardless of if $\varepsilon_{p}<\varepsilon_{m}$ or $\varepsilon_{p}>\varepsilon_{m}$ meaning these equations remain independent of the mixture components. Figure 2.13 shows a representative illustration of the upper bound (left) and lower bound (right).
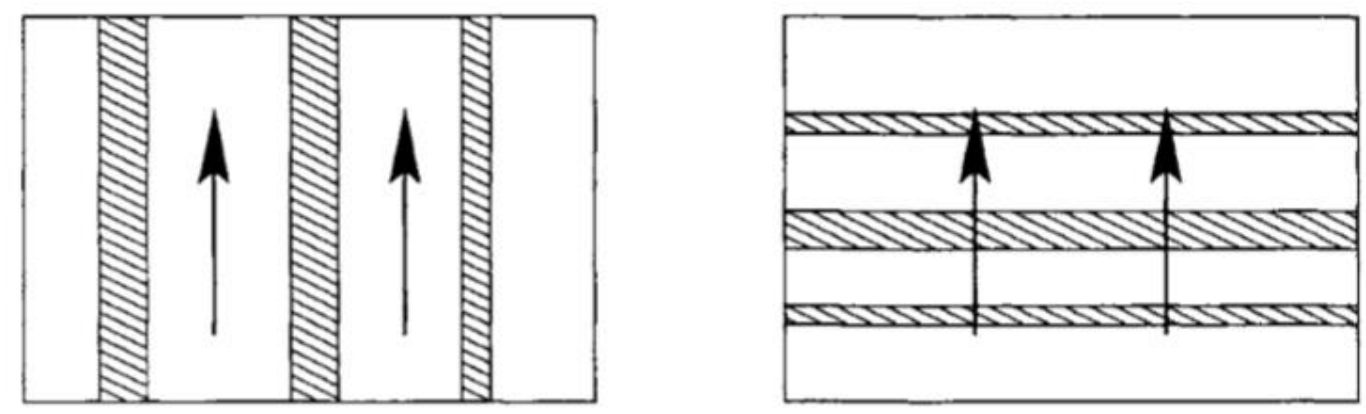

Figure 2.13: The maximum effective permittivity for a given volume fraction of inclusions manifest if the inclusions are in the direction of the flux as shown on the left side. The minimum effective permittivity for a given volume fraction of inclusions corresponds to when the flux is forced to pass through the phase with lower permittivity ${ }^{55}$.

\subsubsection{Hashin-Shtrikman Bounds}

Stricter bounds then the Winer bounds are available in literature, termed the HashinShtrikman bounds. This allows for researchers to get a better idea of the upper and lower limit of the dielectric mixture. An establishment of variational theorems that can then be used to derive limits for permittivity of a mixture is accomplished using these bounds ${ }^{1,27}$. equations 28 and 29 are the upper and lower limit of the Hashin-Shtrikman Bounds, they both assume isotropy in two spatial dimensions ${ }^{27}$. The Hashin-Shtrikman bounds and the Wiener bounds for a dielectric 
inclusion of 10 are shown in Figure 2.14, this helps to illustrate how much different the structure of the Hashin-Shtrikman Bounds are from the Wiener bounds.

$$
\begin{gathered}
\varepsilon_{\text {mix }(\max )}=\varepsilon_{m}+\frac{V_{p}}{\frac{1}{\varepsilon_{p}-\varepsilon_{m}}+\frac{V_{m}}{2 \varepsilon_{m}}} \\
\varepsilon_{\text {mix (min) }}=\varepsilon_{p}+\frac{V_{m}}{\frac{1}{\varepsilon_{m}-\varepsilon_{p}}+\frac{V_{p}}{2 \varepsilon_{p}}}
\end{gathered}
$$

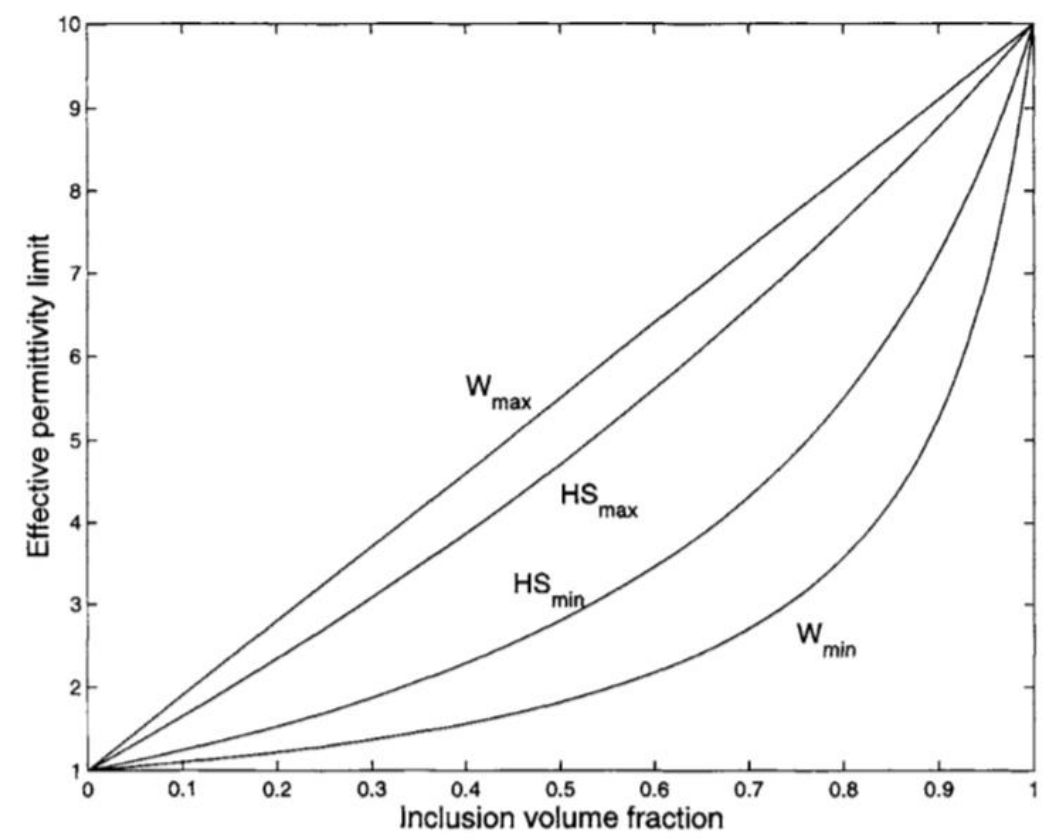

Figure 2.14: Wiener bounds and Hashin-Shtrikman bounds for dielectric constant of a mixture with a dielectric inclusion of $10^{27}$. 


\section{CHAPTER 3: EXPERIMENTAL TESING OF POWDERY MATERIALS}

\subsection{Powder Selection}

In the effort to establish general guidelines and best practice methodology this study looked at four different well characterized powdery materials. Silicon Dioxide $\left(\mathrm{SiO}_{2}\right)$, Aluminum Oxide $\left(\mathrm{Al}_{2} \mathrm{O}_{3}\right)$, Cerium Oxide $\left(\mathrm{CeO}_{2}\right)$ and Anatase Titanium Oxide $\left(\mathrm{TiO}_{2}\right)$. These powders were all commercially available and well understood homogenous powders. The powders were ideal for this study because of their widely varying frequency independent dielectric constant from $3.5-75^{61}$, $62,63,64,65$. It was important to select powders whose dielectric constants were frequency independent in the microwave range to remove additional variables. The variability of the dielectric constants within the dialectic bounds would have resulted in added complexity when sorting through data to determine the accuracy of the mixture equations.

Table 3.1: Powders used in the study to determine the accuracy of the mixture equations for twophase homogenous composites. Associated relevant information such as the supplier, density and dielectric constant.

\begin{tabular}{|c|c|c|c|c|}
\hline Powder & Supplier & Density $\left(\mathbf{g} / \mathbf{c m}^{\mathbf{3}}\right)$ & $\begin{array}{c}\text { Particle Size } \\
(\boldsymbol{\mu m})\end{array}$ & $\begin{array}{c}\text { Dielectric } \\
\text { Constant }\end{array}$ \\
\hline $\mathrm{SiO}_{2}$ & $\begin{array}{c}\text { Sigma-Aldrich at } \\
99.9 \% \text { purity }\end{array}$ & 2.20 & 67 & 3.5 \\
\hline $\mathrm{Al}_{2} \mathrm{O}_{3}$ & $\begin{array}{c}\text { Inframat } \\
\text { Advanced } \\
\text { Materials at } \\
99.7 \% \text { purity }\end{array}$ & 4.00 & 45 & 9.8 \\
\hline $\mathrm{CeO}_{2}$ & $\begin{array}{c}\text { Alfa Aesar at } \\
99.5 \% \text { purity }\end{array}$ & 7.22 & 67 & 21.3 \\
\hline $\mathrm{TiO}_{2}$ & $\begin{array}{c}\text { Acros Organics at } \\
99.5 \% \text { purity }\end{array}$ & 4.23 & 67 & 70 \\
\hline
\end{tabular}

\subsection{Sample Preparation}

\subsubsection{Casting Mold Creation}

In order to utilize the benefits of the paraffin composite method the two-phase composite has to fill all the voids in a given volume. To accomplish this a casting mold needed to be created in the form of an inverted test cell. Measurements of the test cell were preformed using calibers to get accurate measurements. The inner test cell conductor was measured and found to have a diameter of $3 \mathrm{~mm}$ while the inner diameter of the outer conductor had a diameter of $7 \mathrm{~mm}$. Using this information, a CAD file was designed that would allow for a liquid mixture to be cast and cured into the proper design. The CAD design is shown in Figure 3.1 A, it was made using a formlabs 3D printer, a final product with a cured composite is also shown in Figure 3.1 B. Not pictured is the outer sleeve that has an inner diameter of $7 \mathrm{~mm}$ to match that of the coaxial airline. 


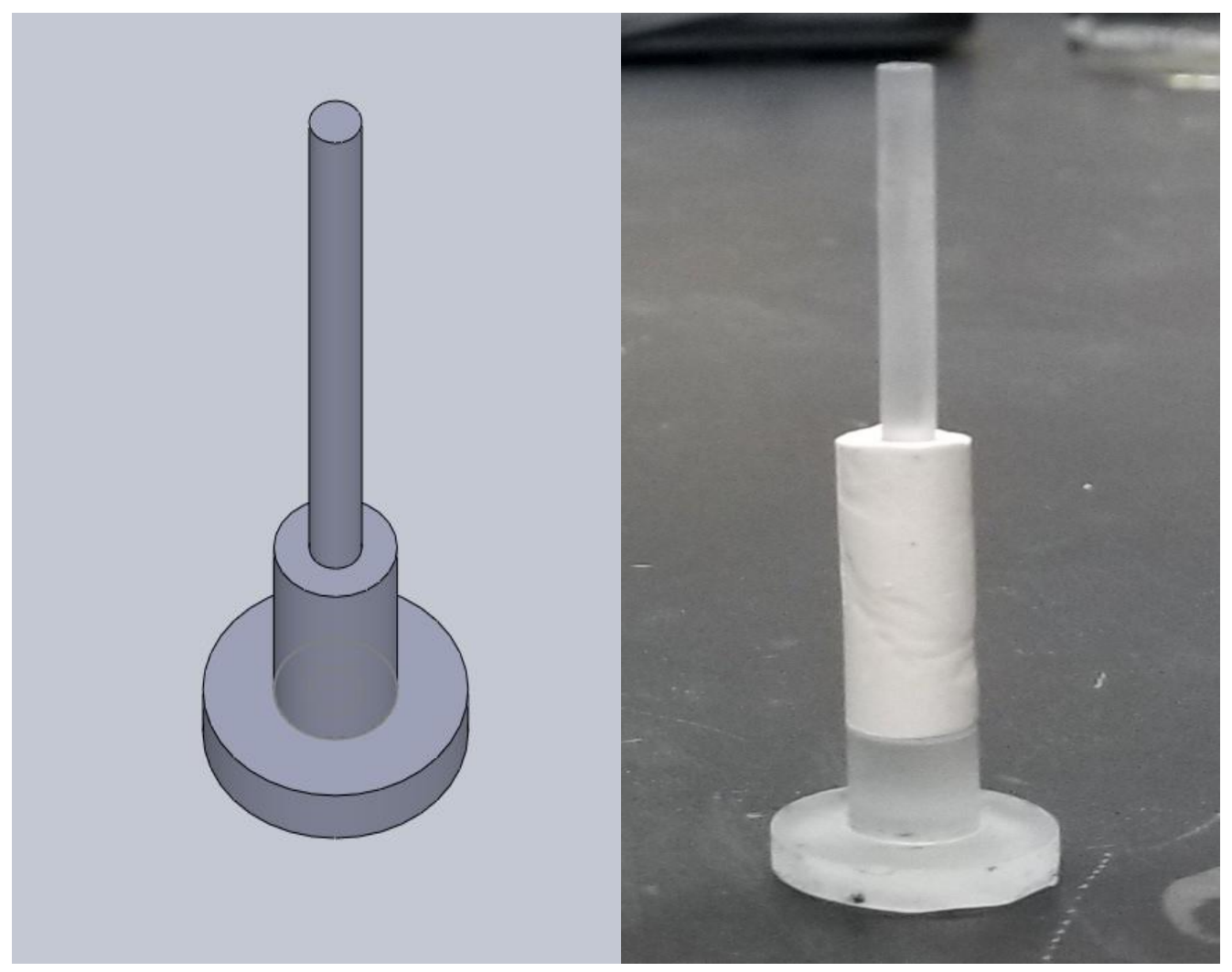

(a)

(b)

Figure 3.1: Subfigure a: 3-D rendering of casting mold for paraffin composite mixtures. The molds are reusable and provide consistency from batch to batch production. Subfigure $b$ the final 3D printed product with a composite mold attached for visualization.

\subsubsection{Volume Loading Selection}

Many studies in literature use different volume loadings of powder with no justification for their choice, this study looks to find a provide a methodology for comparison of these values. To this aim this study chose to look at four different volume loadings of powders, 5\%, 10\%, 20\%, and $30 \%$. These different volume percentages will also provide insight on any thresholds that might exist for this methodology, such as percolation and interparticle effect thresholds ${ }^{192}$. In order to get the correct volume loadings of powder and paraffin a corresponding weight percentage needed to be measured out. Using Equation 31 a volume percentage can be turned into weight percentage and measured out on a scale. Where $\rho_{m}$ and $\rho_{p}$ are the density of the paraffin matrix and the density of the powder respectively.

$$
\text { MasS }_{\%_{\text {Powder }}}=\left(1+\frac{V_{m} \rho_{m}}{V_{p} \rho_{p}}\right)^{-1} * 100
$$

\subsubsection{Composite Mixing}

To create the composite the solid powders needed to be mixed with a liquid paraffin substance at the desired volume loading. The first step is to decide on the volume loading that is 
desired and measure out the corresponding weight percentage using Equation 30. Once the paraffin and powder has been measured they can be combined into a single beaker and placed into a water bath that has been heated to $70{ }^{\circ} \mathrm{C}$. Once the paraffin has melted and is completely liquid mechanical agitation can be used to mix the substances together. For particular viscous substances a sonic wand can be used to help ensure thorough mixture of the solution.

\subsubsection{Curing Process}

Once mixed the solution can be cast into the prepared casting molds up to four per batch. Four is the upper limit when using the water bath method to heat the paraffin as it will start to solidify as soon as it gets to a temperature of $55^{\circ} \mathrm{C}$ or below ${ }^{53}$. The molds are filled to the top and allowed to cool at room temperature for 7 minutes until they become a complete solid again. Once solidified the metal sleeve surrounding the casting mold is removed by slowly twisting it around the sample to break any seals that may have formed whilst also pulling the sleeve off. Once the sleeve is removed the same twisting and pulling process is repeated for the sample to remove it from the center parturition. Once removed the sample can be cut to any desired length, with this study focusing on $10 \mathrm{~mm}$ sample length plugs.

\subsection{Testing Parameters}

Dielectric testing is a high precision measurement technique that is susceptible to high amounts of noise and instability. To reduce these errors careful control of the testing parameters is required. The experimentation in this study was done using a $0.70 \mathrm{~cm}$ diameter coaxial airline (HP model no. 85051-60010) connected to a Keysight N5231A PNA-L microwave network analyzer known as a VNA. The VNA comes with an extensive calibration kit that allows for a complete tuning of phase ambiguities associated with any dislocation of the calibration planes or coaxial lines. The calibration includes reference shorts and open circuits as well as throughput calibrations to allow for the VNA to read zero across the entire spectrum. The test for this study were taken in ambient conditions at room temperature.

\subsection{Sample Testing}

\subsubsection{Testing Cell Preparation}

Preparing the dielectric testing cell for sample testing is imperative for accurate and repeatable results. A cotton swap that has been dipped into isopropanol can be used to remove any debris or dirt that is inside of the cell. The VNA's APC connector's need to be thoroughly cleaned in the same manner to ensure a clean connection where the connector meats the testing cell. The interface between the connectors and the testing cell are where the S-parameters are measured from, specifically the inner and outer face of the conductors. These are the most important aspects of the VNA to keep clean and well maintained. Once the VNA has been cleaned and prepared it is important to ensure that the calibration is still valid. This can be done by connecting the two-ports together and checking the phase measurement to ensure that it is still at zero.

\subsubsection{Sample Loading in Coaxial Test Cell}

Once the samples have been prepared and the testing setup has been cleaned the sample can be loaded in and tested. The loading process is straight forward with the samples loaded into one end of the coaxial line around the center conductor. Figure 3.2 shows a sample loaded into the coaxial line around the inner conductor. In order for this method to be effective no airgaps can 
exist between the sample and the inner or outer conductor. It is also important to ensure no debris from the paraffin composite are blocking the testing interfaces. Straight smooth edges will allow for more accurate measurements of the S-parameters. Once the sample is loaded compressed air can be used to remove any excess debris from the testing setup.

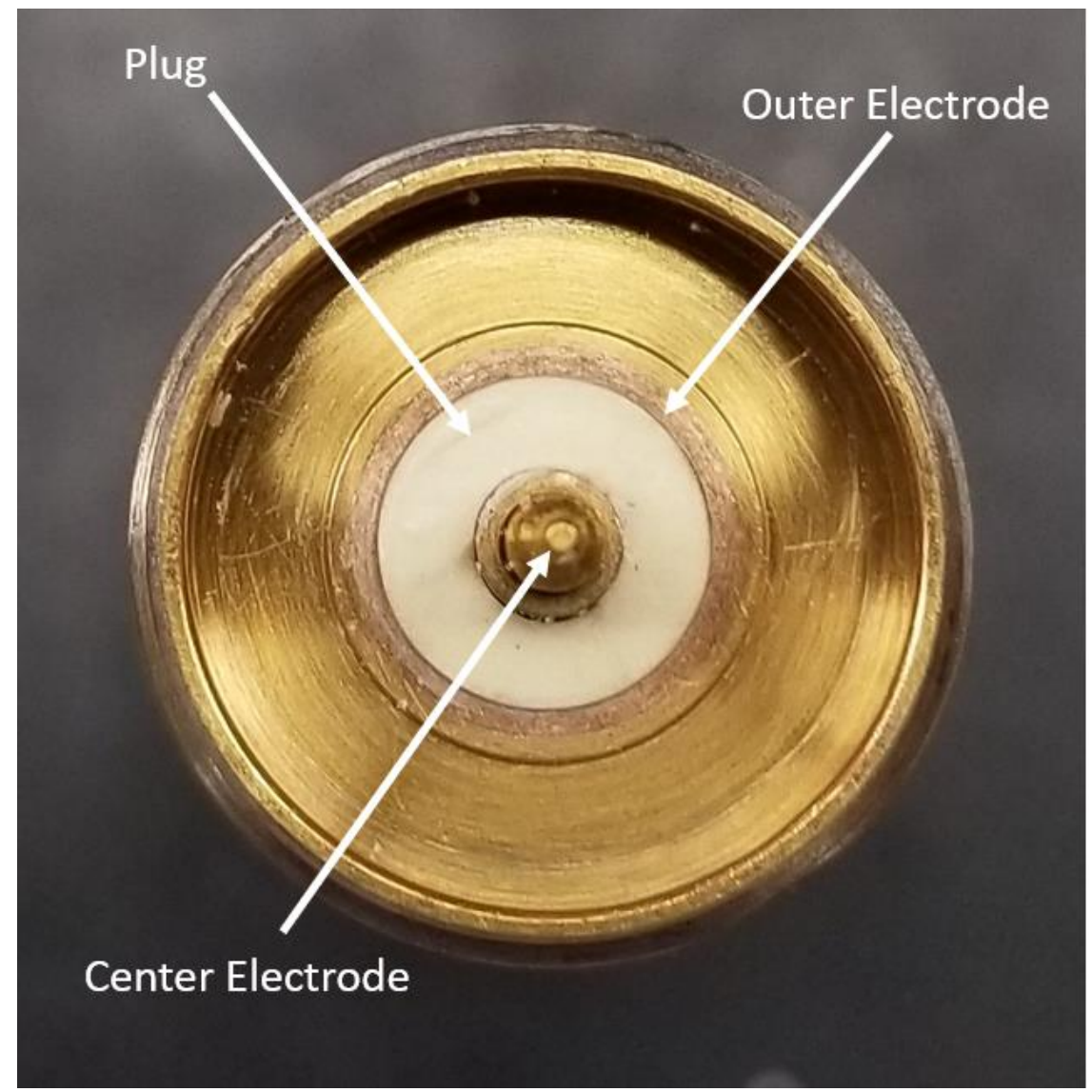

Figure 3.2: Paraffin-powder material composite plug loaded into the precision airline. The plug fills all the space between the center electrode and the outer electrode to ensure no airgaps exist.

\subsubsection{Testing Initiation}

Once the sample has been loaded and is securely in place with no debris a testing procedure can be conducted. Both ends of the airline need to be securely fashioned to the APC connectors in the same orientation that the calibration was performed at. In Figure 3.3 a loaded test cell is shown that is prepared for testing. Once the test cell has been loaded a mathematical model (section 2.3) needs to be selected that transform the S-parameters into the permittivity and permeability. This study used the NRW polynomial method to calculate the permittivity of the composite plugs in order to avoid any discontinuities associated with one half wavelength measurements. Once the model has been selected, the user must define the testing range and number of measurement points. For this study the dielectric properties from 1-10 GHz were investigated using 1601 equally spaced points. Once the model has been selected the VNA will begin measuring and computing until all the designated measurements have been achieved. 


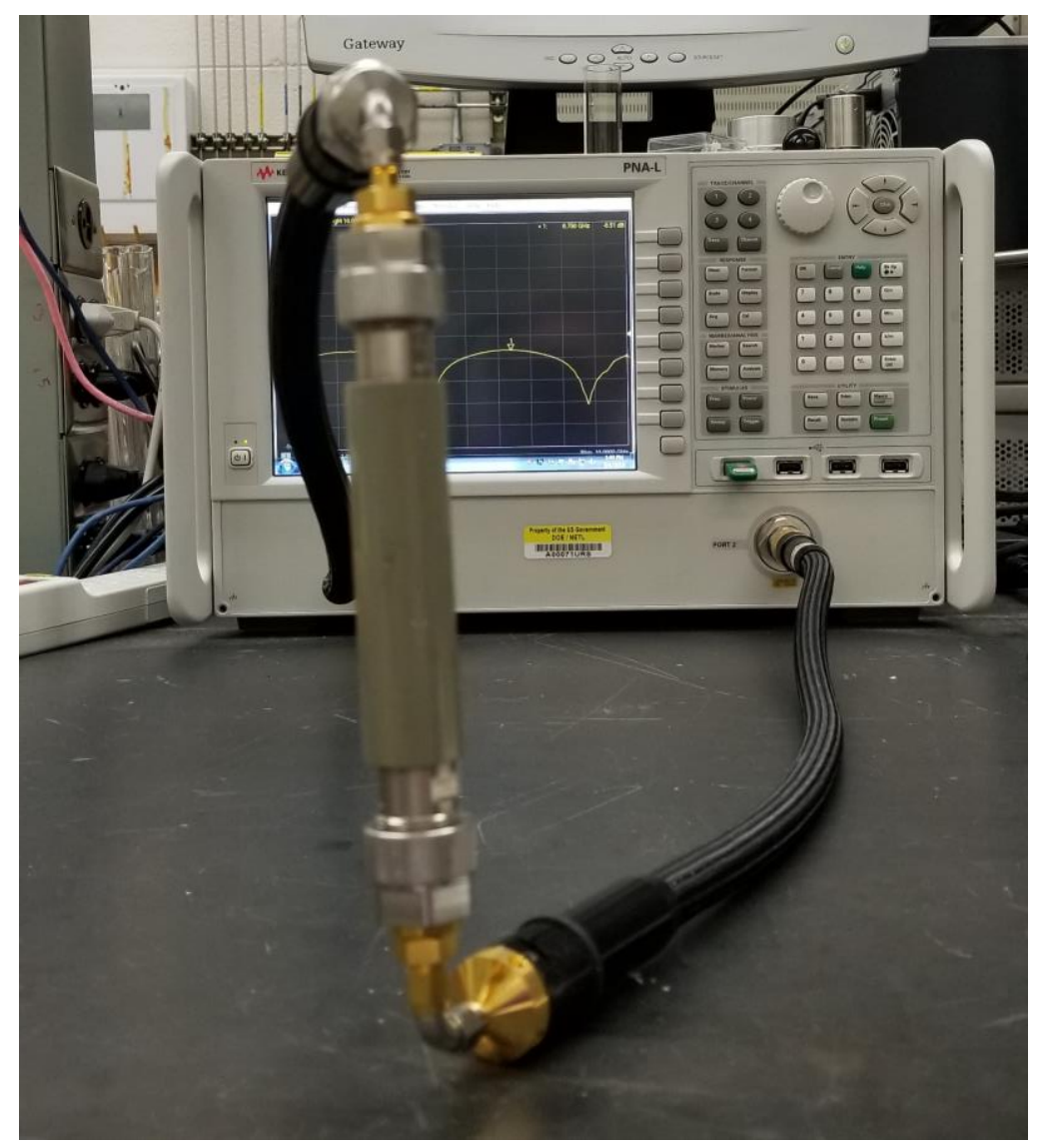

Figure 3.3: VNA setup for high precision coaxial airline testing. Two port VNA with a $10 \mathrm{CM}$ testing line.

\subsection{CT Imaging}

\subsubsection{Dispersion Verification}

For the measurements to be valid for their intended purposes it is paramount that they have a homogenous distribution in the paraffin matrix. To accomplish this CT images were taken from all volume loadings of the composites. The homogeneity of the composites is a requirement in order to make the two-phase dielectric mixture equations valid. An equal dispersion of the powder inside the matrix also will allow for more accurate initial dielectric measurements. This will also increase the accuracy of the mixture equations helping for a determination of the ideal equation to use for these applications. Figure 3.4 shows a $\mathrm{Al}_{2} \mathrm{O}_{3}$ composite that has been $\mathrm{CT}$ scanned and the homogeneity was confirmed using FIJI by analyzing particles per unit volume ${ }^{66}$. The statistical results showed that the volume percentage of particles within the given control volume was on always $\pm 1 \%$ of the desired volume loading. CT scanning also allows for verification that no cracks formed or air bubbles during the curing process. 


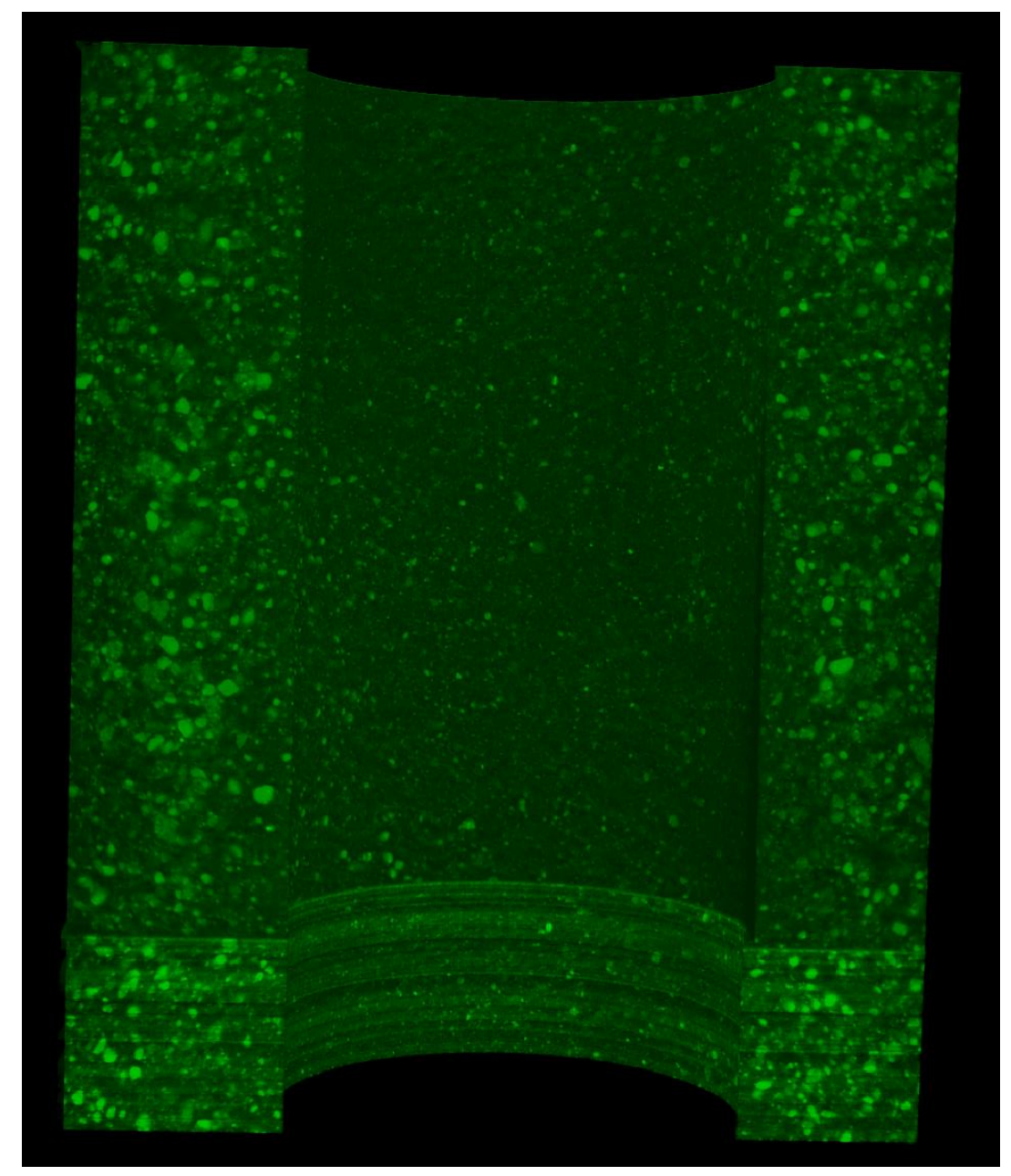

Figure 3.4: Cross sectional view of an $\mathrm{Al}_{2} \mathrm{O}_{3}$ that allows for a visual confirmation of powder distribution within the paraffin matrix. The lines on the bottom are a consequence of beam hardening in the CT scanner ${ }^{66}$.

\subsubsection{Volume Loading Verification}

Another important parameter for the mixture equations is the volume percentages. Since the equations are only reliant upon 4 parameters and two of them are volume loadings it is important to ensure that they are as accurate as possible. FIJI software allows for a volumetric analysis of individual plugs by analyzing the number of particles in the given volume. This was done to ensure that plugs were getting the proper distribution of particles during the mixture and curing stage. The CT scans verified that particles were not conglomerating during any of the stages and overloading any single of the plugs made in a batch. Figure 3.5 shows a CT scan of a $30 \%$ $\mathrm{CeO}_{2}$ composite plug that was used to verify volume loading. Having accurate volume reduces the number of uncontrolled variables in the analysis, allowing for a more complete analysis of the mixture equations. 


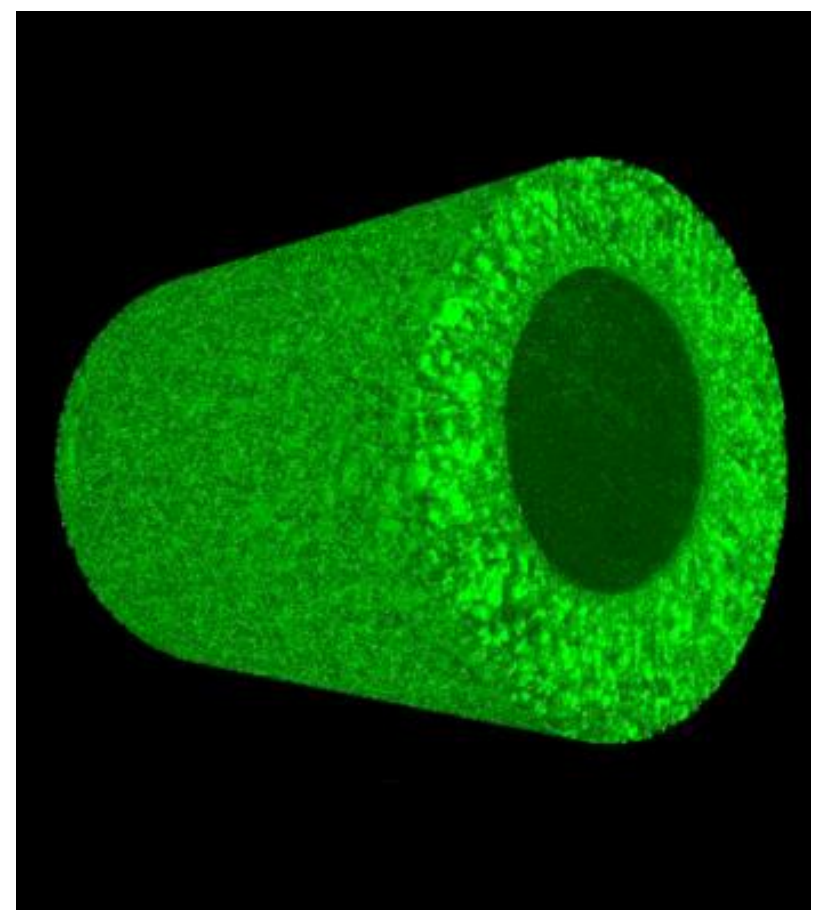

Figure 3.5: 3D rendering from $\mathrm{CT}$ images of a $\mathrm{CeO}_{2} 30 \%$ volume loading composite. Reconstruction was preformed using FIJI and volume loading was verified using there built in software ${ }^{66}$. 


\section{CHAPTER 4: EXPERIMENTAL DIELECTRIC TESTING RESULTS}

For the purpose of investigating the aims of this study, the theoretical output of the mixture equations are compared to the experimentally measured composite values. In this chapter each powder's dielectric constant from literature are used to solve the individual mixture equations for volume loadings of $1-50 \%$. These results are mapped against a scatter plot of the experimental values obtained using the coaxial airline method for volume loadings of 5\%, 10\%, 20\%, and 30\%. The scatter plots are comprised of the 1601 data points taken from 1-10 GHz for each of the 24 individual composites, with 6 composites being tested at each volume loading. The scatter plots show the averaged dielectric constant for each of the volume loading along with an associated tdistribution error bar that shows their range of values corresponding to the unique volume loadings. This is a necessary distribution because of the variability of the dipole-dipole interactions from one measurement to the next ${ }^{55}$.

The absolute percent error for individual volume loadings are calculated to obtain a better understanding of volume loadings effect on each mixture equations precision. The absolute percent error is calculated based on the difference between the average dielectric constant of the mixture and the output of the mixture equation at the corresponding volume loading. This numerical analysis helps to validate what is seen graphically and to help isolate the study of each volume loading. While the graphical results can be used mainly to study the trends of the equations as volume loading increases and to a lesser extent the individual accuracy of each volume loading. With specific focus paid to how the dielectric inclusion ratio $\left(\varepsilon_{p} / \varepsilon_{m}\right)$ for powder to paraffin affects equation accuracy, for both volume loadings and mixture equation.

\subsection{Silicon Dioxide Composite Testing Results}

The theoretical outcomes of the mixture equations as a function of volume loading are graphed against the experimental scatter plot data in Figures 4.1-4.10 for $\mathrm{SiO}_{2}$. The mixture equation uses the dielectric constant of $\mathrm{SiO}_{2}\left(\varepsilon_{p}=3.5\right)$ from literate and the measured dielectric constant of paraffin $\left(\varepsilon_{m}=2.3\right)$ to calculate the output. These compounds were found to have a dielectric inclusion ration of $\varepsilon_{p} / \varepsilon_{m}=1.5$. The absolute percent error between the different measured $\mathrm{SiO}_{2}$ composite and the theoretical output of each mixture equation are represented in Tables 4.1-4.10.

\subsubsection{Theoretical Parallel Mixing Equation Results for $\mathrm{SiO}_{2}$}

The Parallel Mixing equation is the first equation looked at for $\mathrm{SiO}_{2}$, an analysis of its accuracy across volume loadings can be obtained relatively easily. It had a calculated average percent error across all volume loadings of $1.73 \%$ within never more than $3.04 \%$ error for any single volume loading. It should be noted from Figure 4.1 that the equation varies outside of the $\mathrm{t}-$ distribution at high volume loadings (>20\%) Even though when the prediction does vary outside of the t-distribution at high volume loadings the absolute error still remains less than $5 \%$. 
Nevertheless, because it is not within the distribution of the scattering data this equation can be rejected as a viable option for this low dielectric inclusion ratio at high volume loadings.

Table 4.1: Absolute Percent Error for $\mathrm{SiO}_{2}$ composites using Parallel Mixing equation.

\begin{tabular}{|l|l|l|l|l|}
\hline $\mathrm{SiO}_{2}$ & $\mathbf{5 \%}$ VL & $\mathbf{1 0 \%}$ VL & $\mathbf{2 0 \%}$ VL & 30\% VL \\
\hline Percent Error & $1.07 \%$ & $2.13 \%$ & $0.69 \%$ & $3.04 \%$ \\
\hline
\end{tabular}

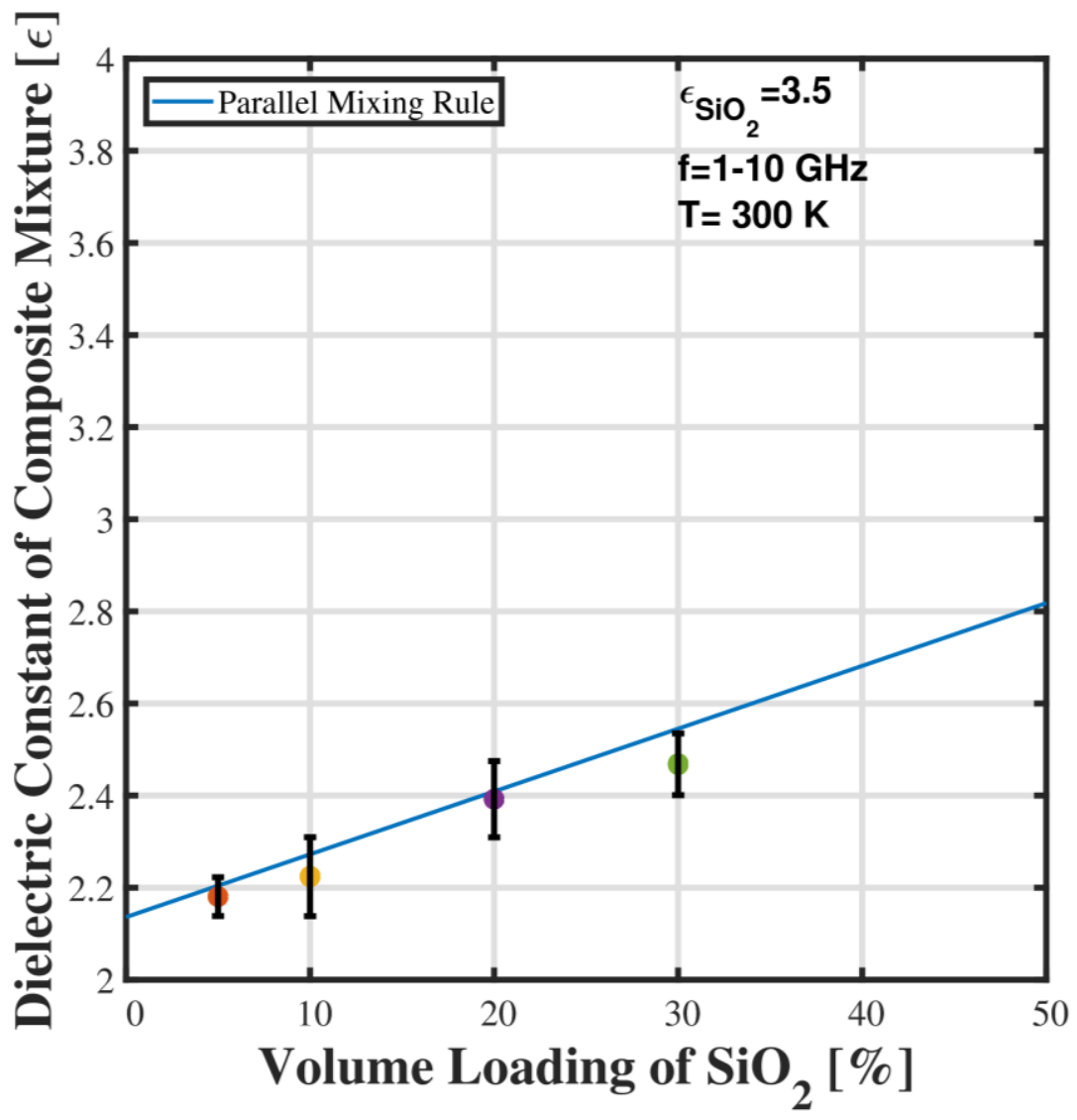

Figure 4.1: Theoretical Parallel mixture equation for $\mathrm{SiO}_{2}$ mapped against experimental scatter plot data for four different volume loadings. 


\subsubsection{Theoretical Series Mixing Equation Results for $\mathrm{SiO}_{2}$}

The next equation investigated is the series mixing equation for its validity in predicting the dielectric constant of the $\mathrm{SiO}_{2}$ composite. A visual analysis of the accuracy of the equation can be quickly surmised using Figure 4.2. This analysis can be quantified using Table 4.2, along with a more details about the individual volume loadings accuracies. The average percent error of the Series mixing equation across all volume loadings is $1.36 \%$, similar to that of previous equation. Unlike the Parallel Mixture equation, at higher volume loadings (>20\%) the Series Mixture equation intersects within the t-distribution. A comparison of Table 4.2 reveals that this equation is much more accurate at low volume loadings $(<20 \%)$ than it is at higher volume loadings.

Table 4.2: Absolute Percent Error for $\mathrm{SiO}_{2}$ composites using Series Mixing equation.

\begin{tabular}{|l|l|l|l|l|}
\hline $\mathrm{SiO}_{2}$ & $\mathbf{5 \%}$ VL & $\mathbf{1 0 \%}$ VL & $\mathbf{2 0 \%}$ VL & 30\% VL \\
\hline Percent Error & $0.09 \%$ & $0.06 \%$ & $3.26 \%$ & $2.03 \%$ \\
\hline
\end{tabular}

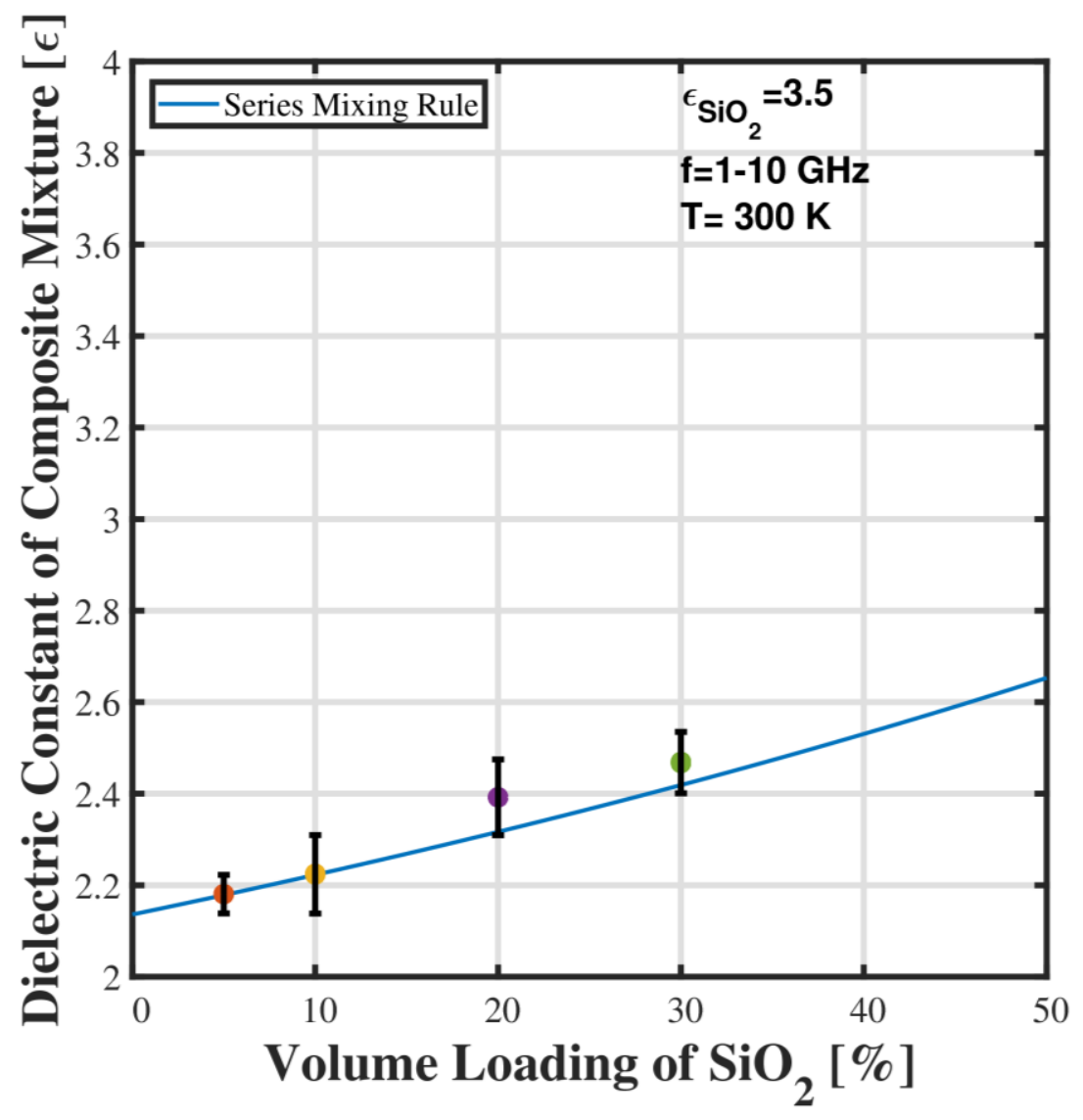

Figure 4.2: Theoretical Series mixture equation for $\mathrm{SiO}_{2}$ mapped against experimental scatter plot data for four different volume loadings. 


\subsubsection{Theoretical Logarithmic Mixing Equation Results for $\mathrm{SiO}_{2}$}

The Logarithmic Mixing equation is evaluated for its ability to map the increasing dielectric constant of the $\mathrm{SiO}_{2}$ loaded composite. The graphical information in Figure 4.3 clearly indicates that the logarithmic mixture equation is not suitable for this dielectric inclusion ratio. At no point does the theoretical output come close to the experimental distribution. A comparison with Table 4.3 shows quantitively that at each volume loading measured the Logarithmic Mixing equation massively miscalculates the dielectric constant of the mixture. The average percent error across all volume loadings for the Logarithmic Mixing equation is $25.97 \%$ which is high in comparison to the other equations for this powder.

Table 4.3: Absolute Percent Error for $\mathrm{SiO}_{2}$ composites using Logarithmic Mixing equation.

\begin{tabular}{|l|l|l|l|l|}
\hline $\mathrm{SiO}_{2}$ & $\mathbf{5 \%}$ VL & $\mathbf{1 0} \%$ VL & $\mathbf{2 0 \%}$ VL & 30\% VL \\
\hline Percent Error & $30.14 \%$ & $28.57 \%$ & $23.33 \%$ & $21.84 \%$ \\
\hline
\end{tabular}

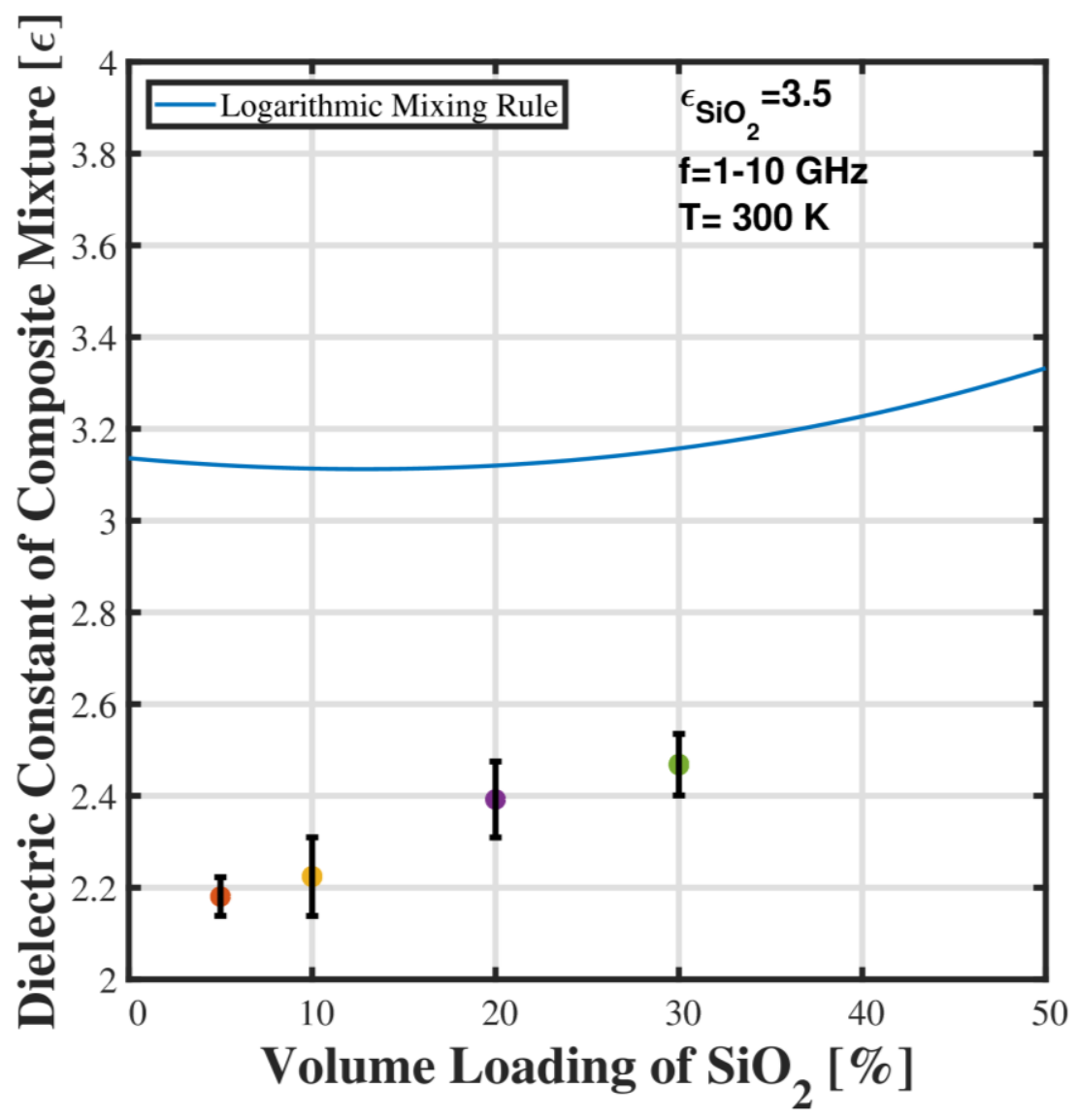

Figure 4.3: Theoretical Logarithmic mixture equation for $\mathrm{SiO}_{2}$ mapped against experimental scatter plot data for four different volume loadings. 


\subsubsection{Theoretical Lichtenecker Mixing Equation for $\mathrm{SiO}_{2}$}

The Lichtenecker mixing equation is also evaluated for its ability to accurately predict the dielectric constant of a $\mathrm{SiO}_{2}$ composite as a function of volume loading. Like most of the other equations the Lichtenecker has a linear relationship with volume loading. The graph reveals that the Lichtenecker mixing equation passes within the t-distribution for all volume loadings shown. A comparison of Table 4.4 shows that the percent error is never greater than $1.46 \%$ for any volume loading, with an average percent error of $0.78 \%$. This low error percentage indicates that the equation can most likely accurately follow the increasing trend of this compound as it approaches a volume loading of $100 \%$. Even though the actual relationship of dielectric constant to volume loadings isn't completely linear.

Table 4.4: Absolute Percent Error for $\mathrm{SiO}_{2}$ composites using Lichtenecker Mixing equation.

\begin{tabular}{|l|l|l|l|l|}
\hline $\mathrm{SiO}_{2}$ & $\mathbf{5 \%}$ VL & $\mathbf{1 0 \%}$ VL & $\mathbf{2 0 \%}$ VL & $\mathbf{3 0 \%}$ VL \\
\hline Percent Error & $0.41 \%$ & $0.90 \%$ & $1.46 \%$ & $0.37 \%$ \\
\hline
\end{tabular}

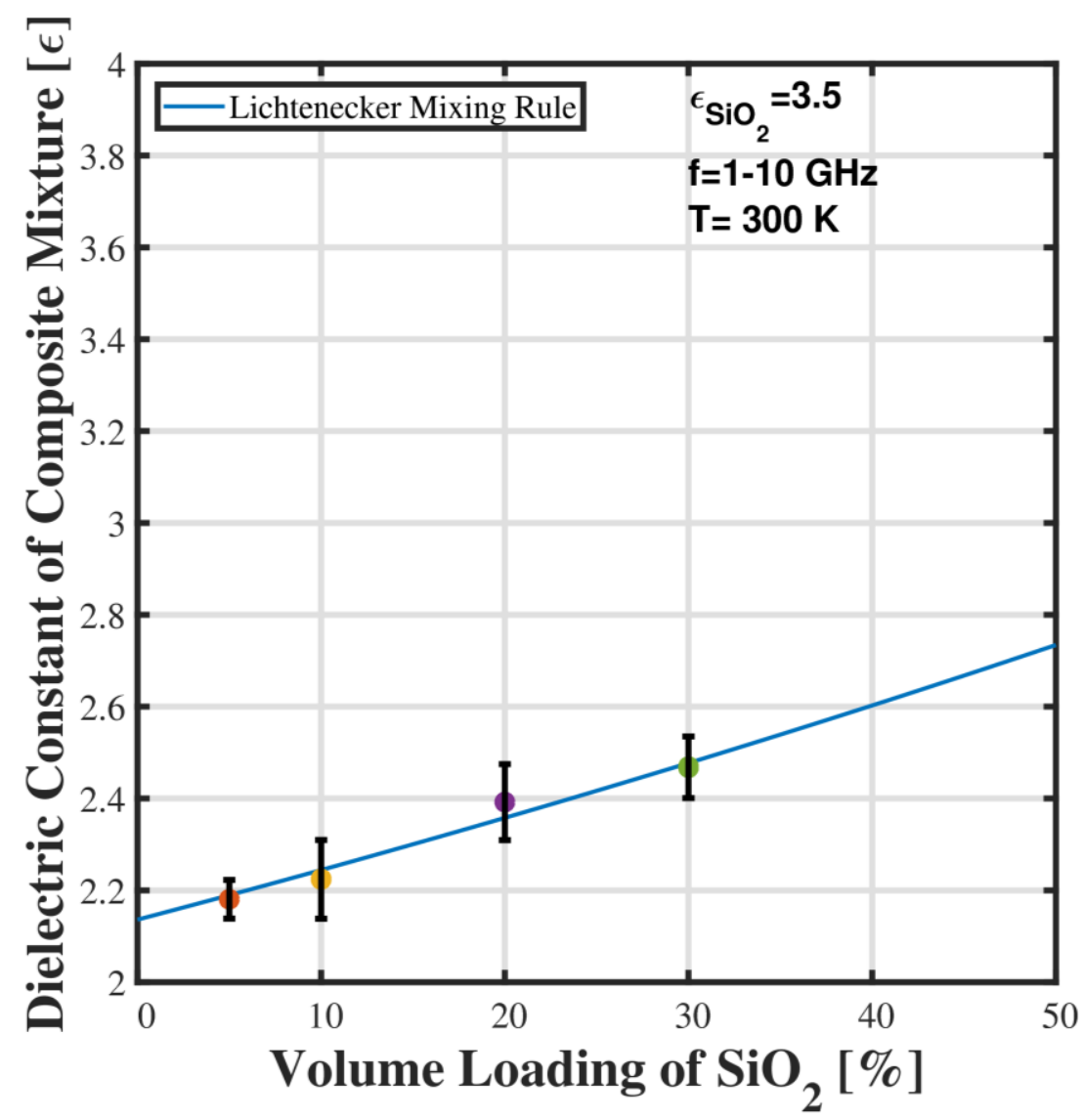

Figure 4.4: Theoretical Lichtenecker mixture equation for $\mathrm{SiO}_{2}$ mapped against experimental scatter plot data for four different volume loadings. 


\subsubsection{Theoretical Looyenga Mixing Equation for $\mathrm{SiO}_{2}$}

The Looyenga mixing rule is analyzed for its applicability in the purposes of this study. Table 4.5 shows that the accuracy of the Looyenga mixing equation never gives more than $1.24 \%$ error, with the overall error $0.97 \%$ for all volume loadings. While this is slightly higher than the previous equation it is better than the others so far reviewed in this study. These results can be clearly verified by a comparison with Figure 4.5 . The mixture equation once again passes through the t-distribution for all measured volume loadings providing an indicator that this might be another equation that is suitable for low dielectric inclusion ratios across all volume loadings.

Table 4.5: Absolute Percent Error for $\mathrm{SiO}_{2}$ composites using Looyenga Mixing equation.

\begin{tabular}{|l|l|l|l|l|}
\hline $\mathrm{SiO}_{2}$ & $\mathbf{5 \%}$ VL & $\mathbf{1 0 \%}$ VL & $\mathbf{2 0 \%}$ VL & $\mathbf{3 0 \%}$ VL \\
\hline Percent Error & $0.61 \%$ & $1.28 \%$ & $0.78 \%$ & $1.24 \%$ \\
\hline
\end{tabular}

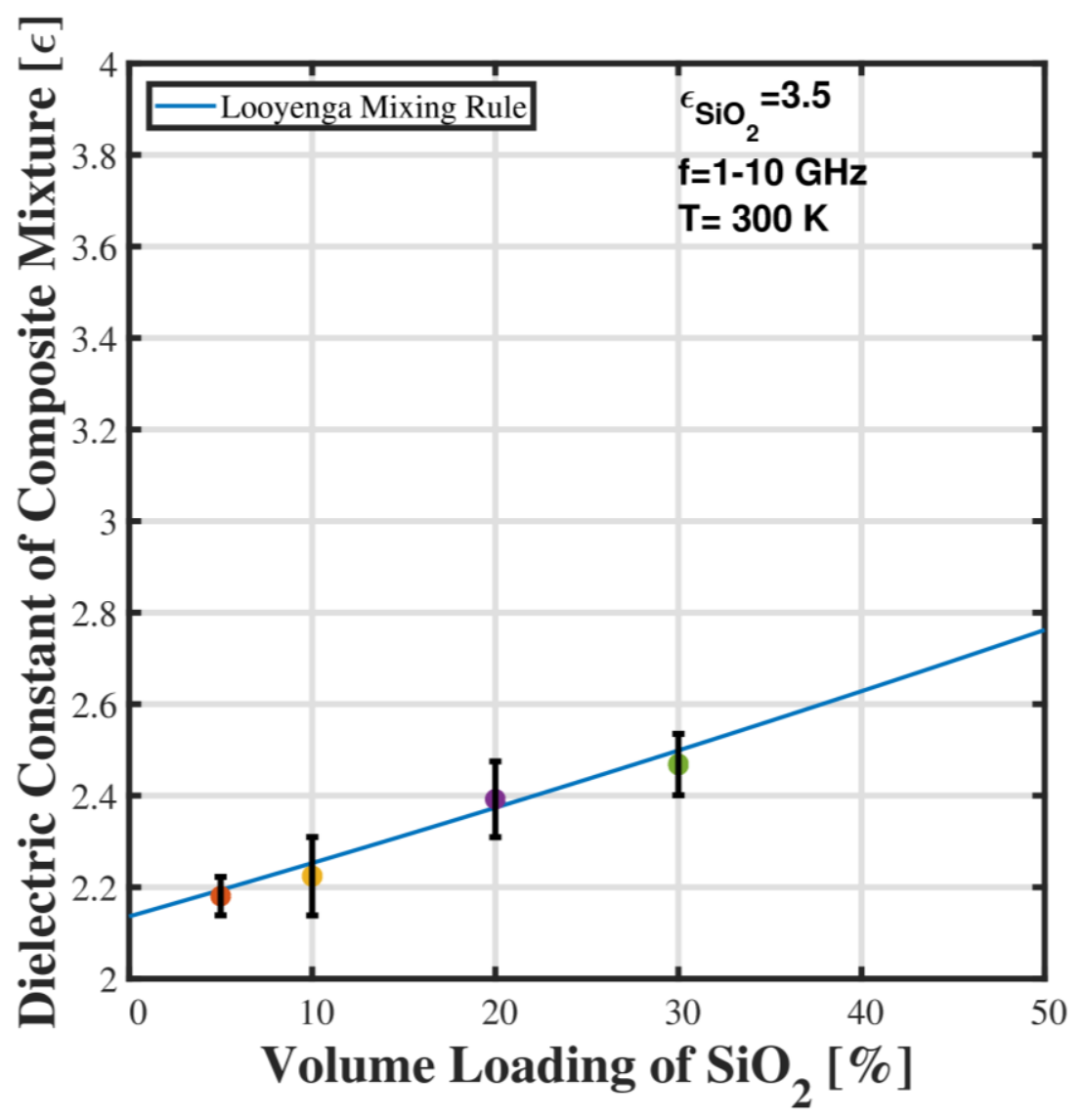

Figure 4.5: Theoretical Looyenga mixture equation for $\mathrm{SiO}_{2}$ mapped against experimental scatter plot data for four different volume loadings. 


\subsubsection{Theoretical Birchak Mixing Equation for $\mathrm{SiO}_{2}$}

Continuing the evaluation of the two-phase homogenous mixture equation's the Birchak mixing equation is evaluated across the different volume loadings. Like most of the other equations with the low dielectric inclusion ratio of $\mathrm{SiO}_{2}$ to paraffin, the Birchak mixing equation is extremely accurate. The Birchak equation never has a percent error of more than $1.68 \%$ error for any single volume loading, with an average percent error of only $1.07 \%$. While still not the most accurate equation discussed in this section, the Birchak equation's accuracy is extremely high for the nearly linear increasing experimental values. Once again this can be easily seen from Figure 4.6, where the Birchak mixing equation never varies outside of the t-distributions. This continues to support the idea that the most mixture equations are applicable at this low dielectric inclusion ratio.

Table 4.6: Absolute Percent Error for $\mathrm{SiO}_{2}$ composites using Birchak Mixing equation.

\begin{tabular}{|l|l|l|l|l|}
\hline $\mathrm{SiO}_{2}$ & $\mathbf{5 \%}$ VL & $\mathbf{1 0 \%}$ VL & $\mathbf{2 0 \%}$ VL & 30\% VL \\
\hline Percent Error & $0.72 \%$ & $1.48 \%$ & $0.42 \%$ & $1.68 \%$ \\
\hline
\end{tabular}

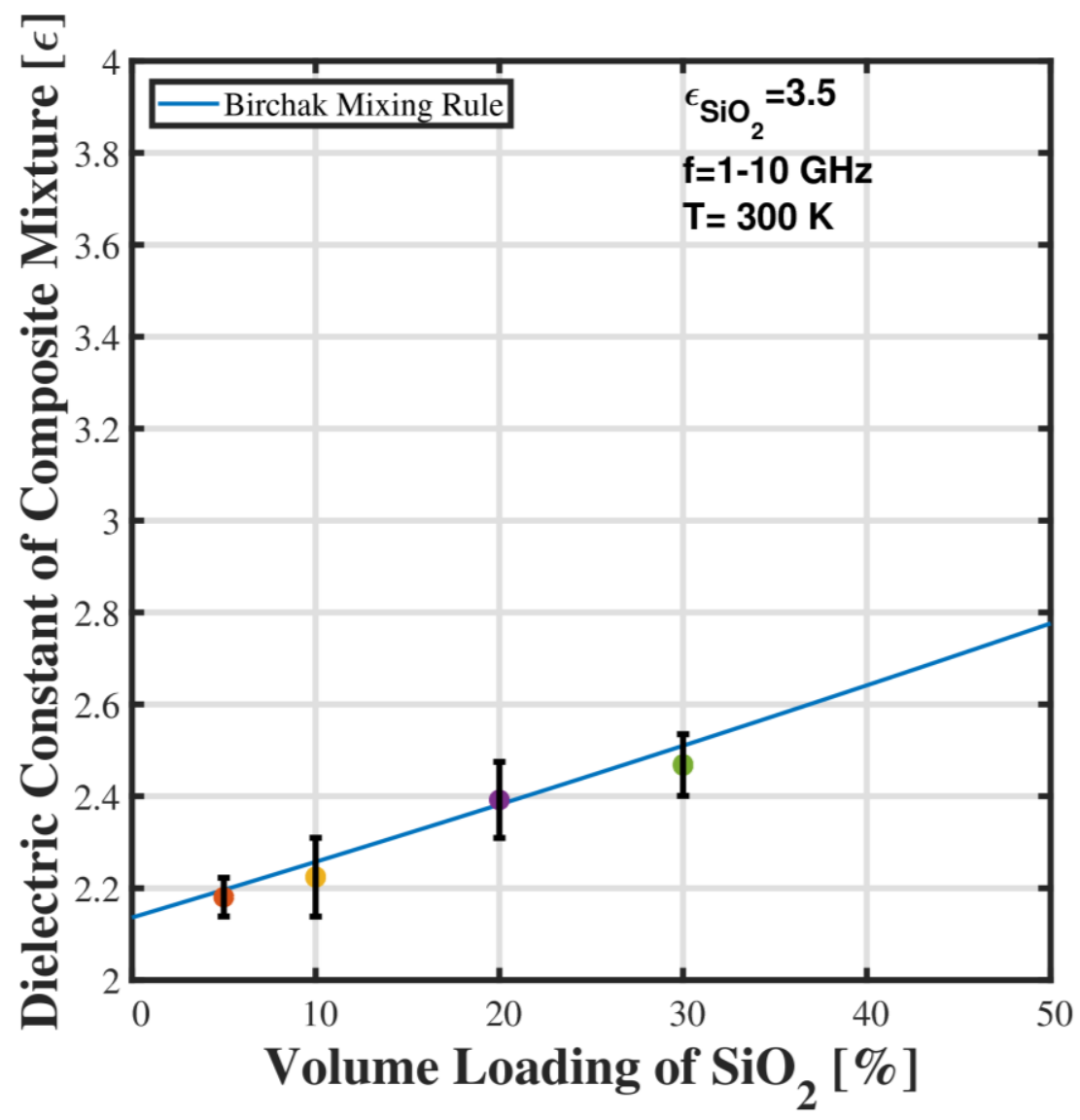

Figure 4.6: Theoretical Birchak mixture equation for $\mathrm{SiO}_{2}$ mapped against experimental scatter plot data for four different volume loadings. 


\subsubsection{Theoretical Poon-Shin Mixing Equation for $\mathrm{SiO}_{2}$}

An evaluation of the performance of the Poon-Shin Mixing equation was completed using experimental results of the previously discussed $\mathrm{SiO}_{2}$-paraffin composites. Like the other equations used for predictive analysis of this low dielectric inclusion ratio, the Poon-Shin mixing equation is very accurate. From Table 4.7 it can be seen that the mixture equation never has an error percentage greater than $1.54 \%$, with an average percent error of $1.01 \%$. An analysis of Figure 4.7 shows that this is another equation that does an excellent job of mapping the growth of the dielectric constant of the compound as a function of volume loading.

Table 4.7: Absolute Percent Error for $\mathrm{SiO}_{2}$ composites using Poon-Shin Mixing equation.

\begin{tabular}{|l|l|l|l|l|}
\hline $\mathrm{SiO}_{2}$ & $\mathbf{5 \%}$ VL & $\mathbf{1 0 \%}$ VL & $\mathbf{2 0 \%}$ VL & $\mathbf{3 0 \%}$ VL \\
\hline Percent Error & $0.58 \%$ & $1.26 \%$ & $0.67 \%$ & $1.54 \%$ \\
\hline
\end{tabular}

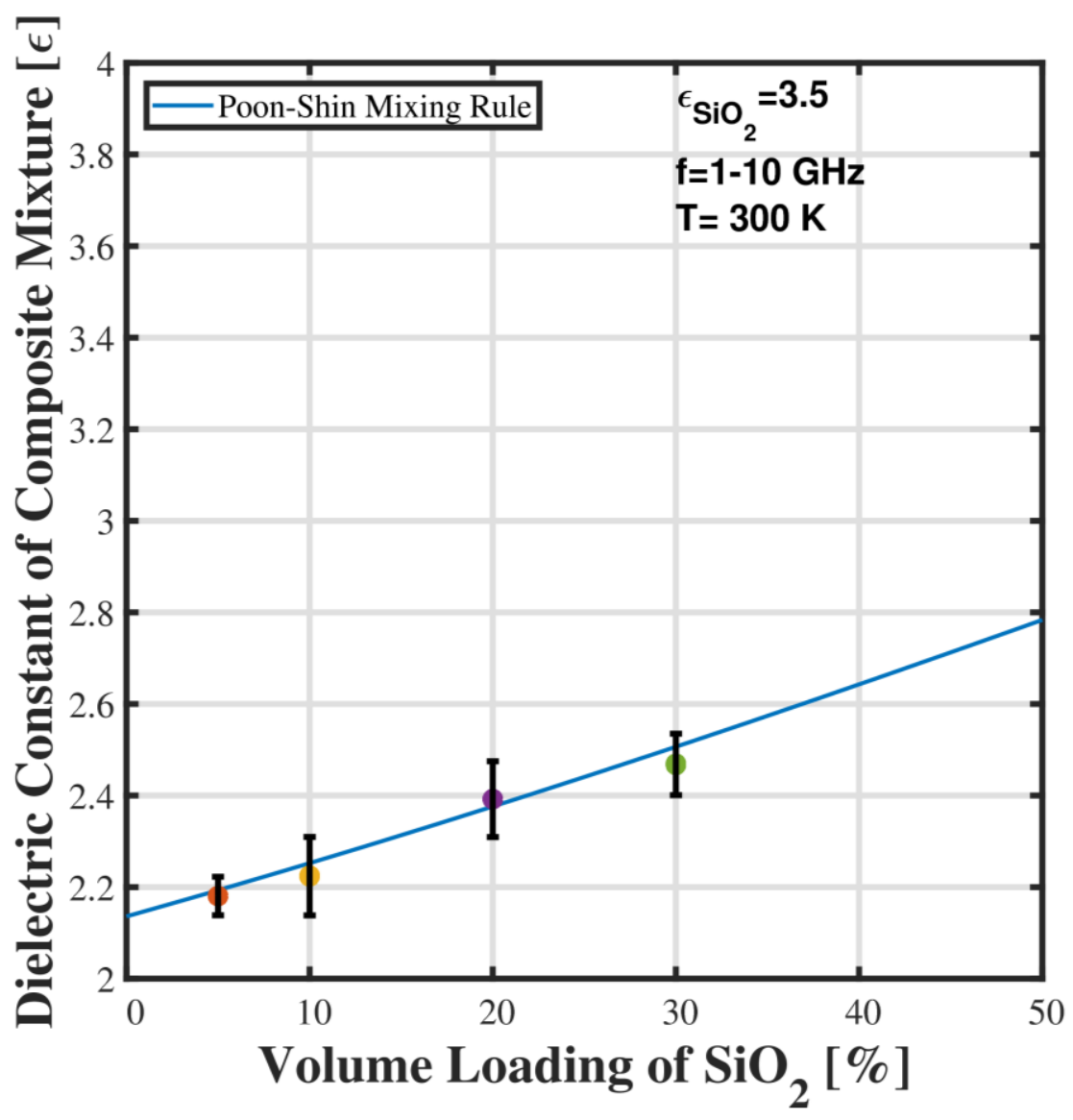

Figure 4.7: Theoretical Poon-Shin mixture equation for $\mathrm{SiO}_{2}$ mapped against experimental scatter plot data for four different volume loadings. 


\subsubsection{Theoretical Effective Medium Theory Mixing Equation for $\mathrm{SiO}_{2}$}

The EMT mixing equation is assessed for its capability to correctly predict the dielectric constant of $\mathrm{SiO}_{2}$ composites. Unlike most of the other equations the EMT has a slightly parabolic relationship with volume loading, this is a factor of the added shape factor variable in the EMT equation. The graph in Figure 4.8, reveals that the EMT mixing equation passes within the tdistribution for all volume loadings shown despite this more parabolic shape. A comparison of Table 4.4 shows that the percent error is never greater than $1.76 \%$ for any volume loading, with an average percent error of $0.94 \%$. This low error percentage indicates that the equation can accurately follow the increasing trend of this compound as it approaches the pure powder value.

Table 4.8: Absolute Percent Error for $\mathrm{SiO}_{2}$ composites using EMT Mixing equation.

\begin{tabular}{|l|l|l|l|l|}
\hline $\mathrm{SiO}_{2}$ & $\mathbf{5 \%}$ VL & $\mathbf{1 0 \%}$ VL & $\mathbf{2 0 \%}$ VL & 30\% VL \\
\hline Percent Error & $0.98 \%$ & $1.76 \%$ & $0.65 \%$ & $0.36 \%$ \\
\hline
\end{tabular}

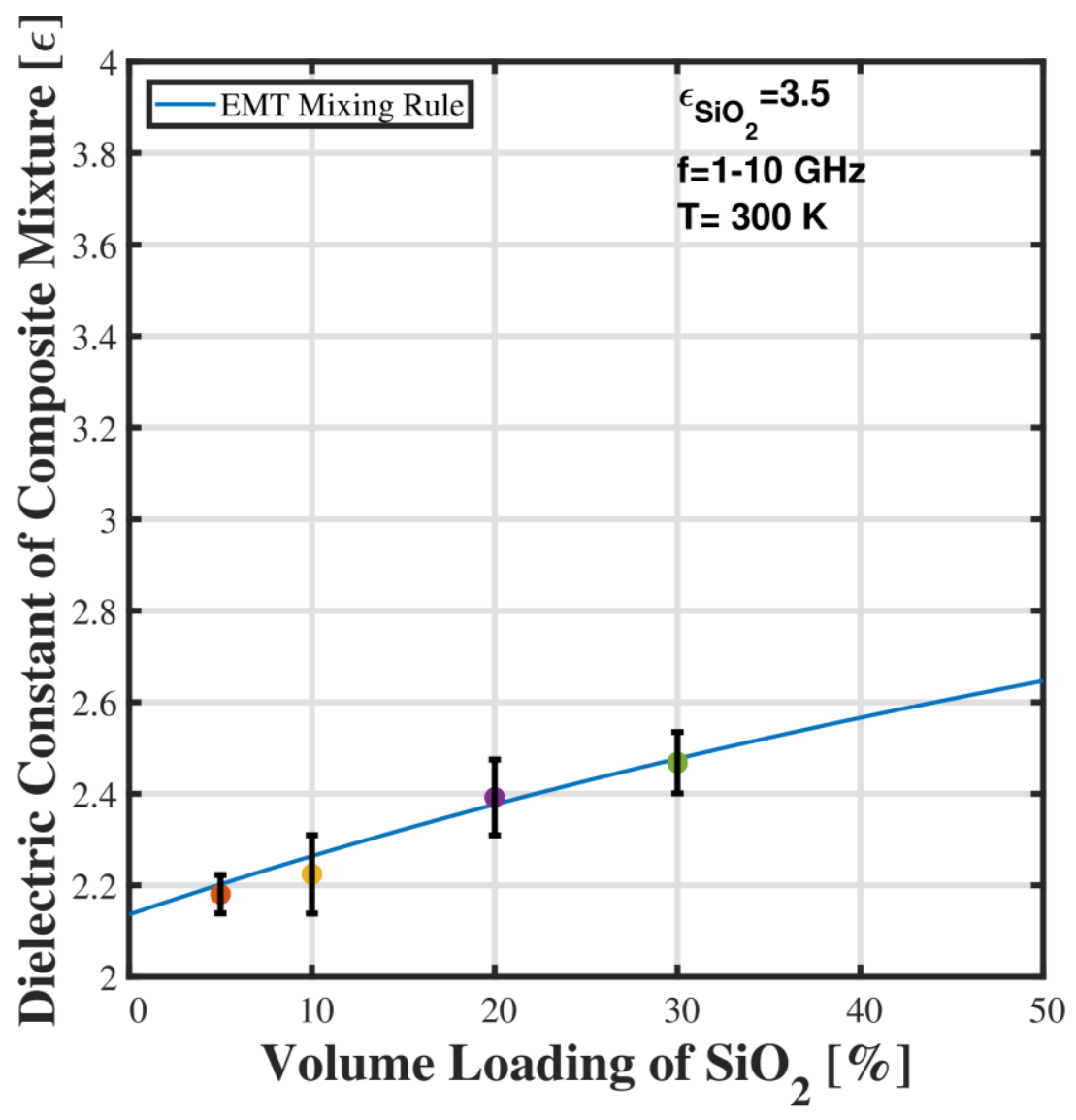

Figure 4.8: Theoretical EMT mixture equation for $\mathrm{SiO}_{2}$ mapped against experimental scatter plot data for four different volume 


\subsubsection{Theoretical Maxwell-Garnett Mixing Equation for $\mathrm{SiO}_{2}$}

The ongoing evaluation of a $\mathrm{SiO}_{2}$ two-phase homogenous composites ability to be mapped using a mixture equation's next uses the Maxwell-Garnet Mixing equation. Comparably to most of the other equations in this section, the Maxwell-Garnett mixing equation is extremely accurate. The Maxwell-Garnett equation never has a percent error of more than $1.18 \%$ error for any single volume loading, with an average percent error of only $0.92 \%$. This follows the same trend as the majority of the equations in this section with little deviation in the mixture equation accuracies being shown.

Table 4.9: Absolute Percent Error for $\mathrm{SiO}_{2}$ composites using Maxwell-Garnett Mixing equation.

\begin{tabular}{|l|l|l|l|l|}
\hline $\mathrm{SiO}_{2}$ & $\mathbf{5 \%}$ VL & $\mathbf{1 0 \%}$ VL & $\mathbf{2 0 \%}$ VL & $\mathbf{3 0 \%}$ VL \\
\hline Percent Error & $0.56 \%$ & $1.18 \%$ & $0.97 \%$ & $0.97 \%$ \\
\hline
\end{tabular}

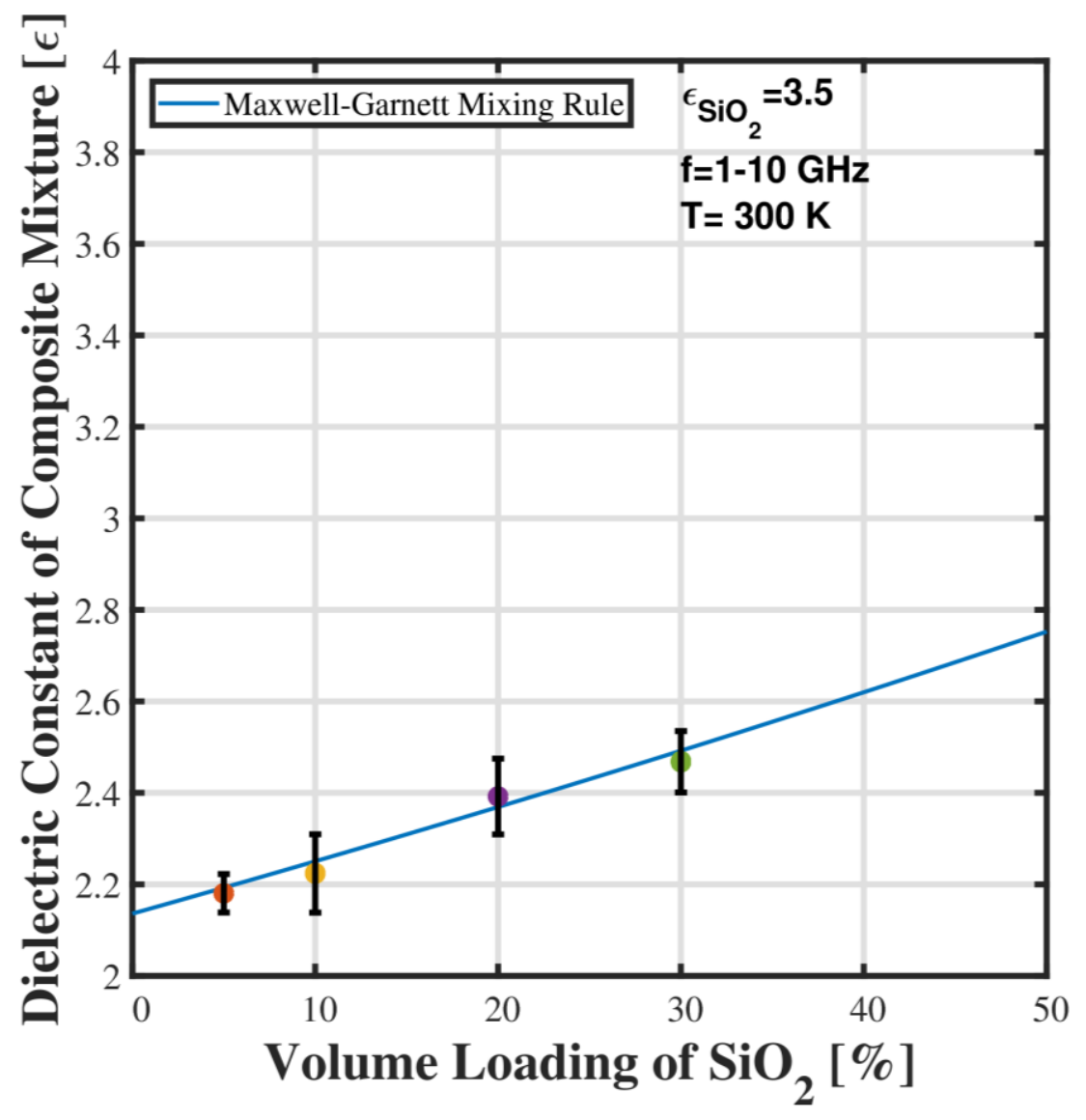

Figure 4.9: Theoretical Maxwell-Garnett mixture equation for $\mathrm{SiO}_{2}$ mapped against experimental scatter plot data for four different volume loadings. 


\subsubsection{Theoretical Jayasundere-Smith Mixing Equation for $\mathrm{SiO}_{2}$}

The final equation examined was the Jayasundere-Smith mixing equation, Table 4.10 shows that the accuracy of the Looyenga mixing equation never becomes more than $2.6 \%$ error. With the overall error $1.18 \%$ for all volume loadings, these results can be clearly clarified by a comparison with Figure 4.10. The mixture equation once again passes through the t-distribution for all measured volume loadings providing an indicator that this might be another equation that is suitable for low dielectric inclusion ratios. However, at high volume loadings $(>30 \%)$ the equation is barely inside the t-distribution, indicating that this equation might not be suitable for high volume loadings.

Table 4.10: Absolute Percent Error for $\mathrm{SiO}_{2}$ composites using Jayasundere-Smith Mixing equation.

\begin{tabular}{|l|l|l|l|l|}
\hline $\mathrm{SiO}_{2}$ & $\mathbf{5 \%}$ VL & $\mathbf{1 0 \%}$ VL & $\mathbf{2 0 \%}$ VL & $\mathbf{3 0 \%}$ VL \\
\hline Percent Error & $0.62 \%$ & $1.42 \%$ & $0.12 \%$ & $2.54 \%$ \\
\hline
\end{tabular}

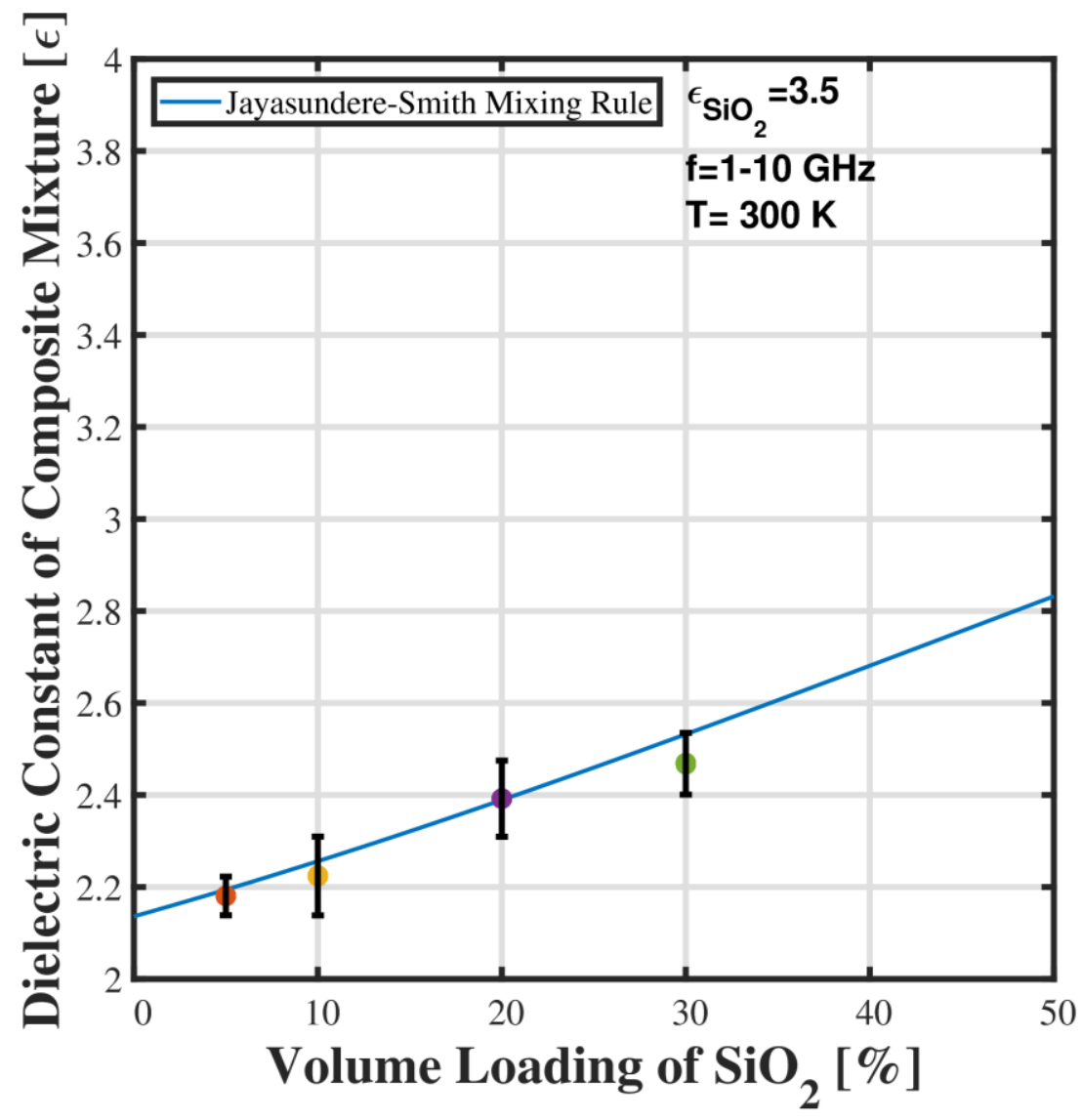

Figure 4.10: Theoretical Jayasundere-Smith mixture equation for $\mathrm{SiO}_{2}$ mapped against experimental scatter plot data for four different volume loadings. 


\subsection{Aluminum Oxide Dioxide Composite Testing Results}

After evaluating all the mixture equations for the low dielectric inclusion ration of 1.5 for $\mathrm{SiO}_{2}$ to paraffin the study next looks at a $\mathrm{Al}_{2} \mathrm{O}_{3}$ as the inclusion powder. The higher dielectric constant of $\mathrm{Al}_{2} \mathrm{O}_{3}\left(\varepsilon_{p}=9.8\right)$ results in a dielectric inclusion ratio of $\varepsilon_{p} / \varepsilon_{m}=4.3$. The theoretical outcomes of the mixture equations as a function of volume loading are graphed against the experimental scatter plot data in Figures 4.11-4.20 for $\mathrm{Al}_{2} \mathrm{O}_{3}$. The absolute percent error between the different measured $\mathrm{Al}_{2} \mathrm{O}_{3}$ composite and the theoretical output of each mixture equation are represented in Tables 4.11-4.20.

\subsubsection{Theoretical Parallel Mixing Equation Results for $\mathrm{Al}_{2} \mathrm{O}_{3}$}

The results of the of the Parallel Mixing equation's ability to predict $\mathrm{Al}_{2} \mathrm{O}_{3}$ compounds dielectric constant are discussed in this section. The Parallel Mixing equation was found to have an average percent error across all volume loadings of $14.82 \%$ this is an increase from the previous constitutive powder by $13.09 \%$. Figure 4.11 and Table 4.11 shows that the parallel mixing rule does a terrible job of predicting the behavior of the composites dielectric constant as volume loading increases. It can be seen from the Figure that the behavior of the measured composite's dielectric constant no longer behaves linearly. This is a direct consequence of the dielectric inclusion ratio. The increase in inclusion ratio corresponds to the large increase in error seen from the previous section.

Table 4.11: Absolute Percent Error for $\mathrm{Al}_{2} \mathrm{O}_{3}$ composites using the Parallel Mixing equation.

\begin{tabular}{|l|l|l|l|l|}
\hline $\mathrm{Al}_{2} \mathrm{O}_{3}$ & $\mathbf{5 \%} \mathbf{~ V L}$ & $\mathbf{1 0 \%}$ VL & $\mathbf{2 0 \%}$ VL & 30\% VL \\
\hline Percent Error & $4.78 \%$ & $7.37 \%$ & $19.44 \%$ & $27.68 \%$ \\
\hline
\end{tabular}




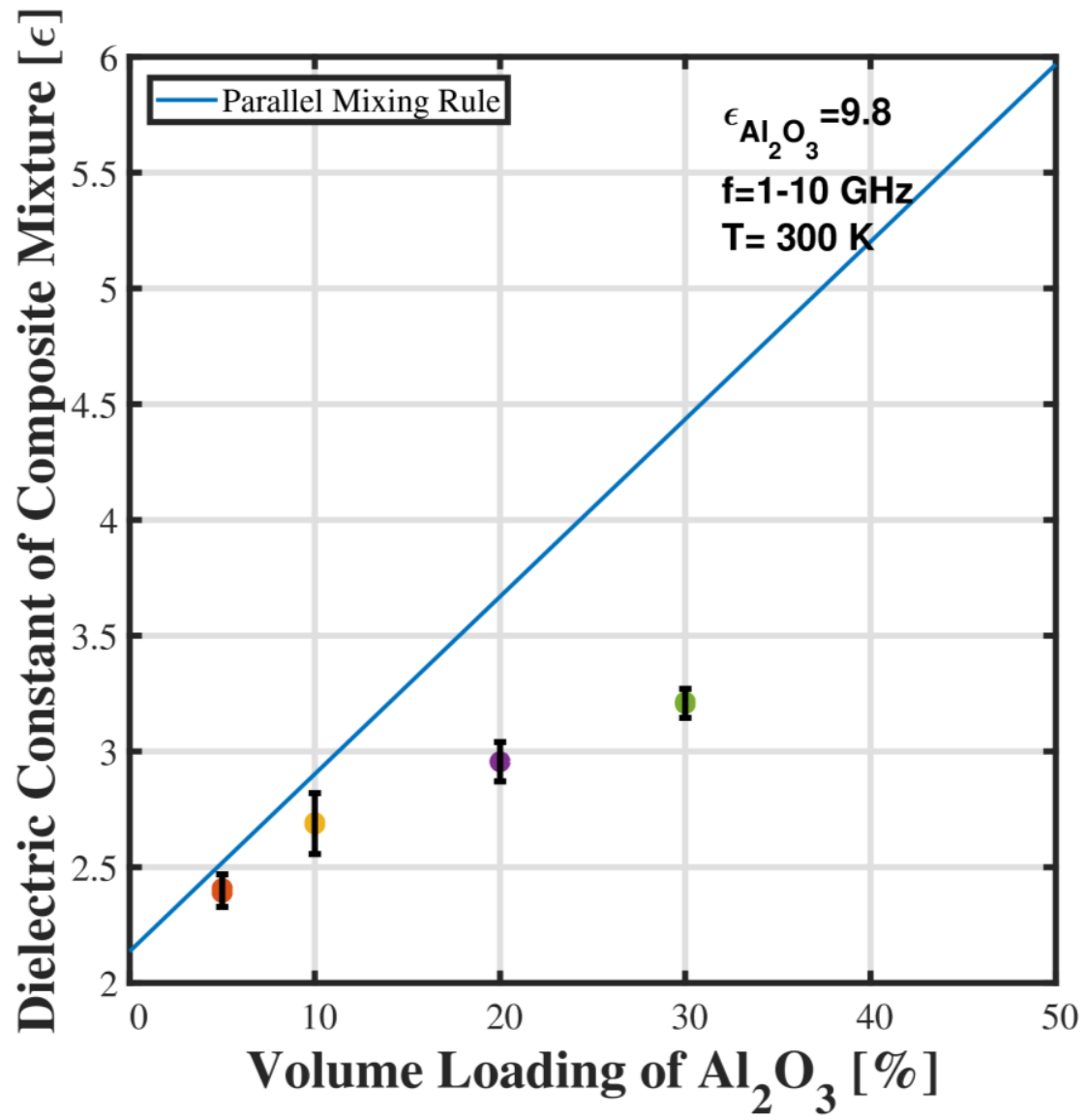

Figure 4.11: Theoretical Parallel mixture equation for $\mathrm{Al}_{2} \mathrm{O}_{3}$ mapped against experimental scatter plot data for four different volume loadings. 


\subsubsection{Theoretical Series Mixing Equation Results for $\mathrm{Al}_{2} \mathrm{O}_{3}$}

The subsequent equation examined is the series mixing equation for its validity in calculating the dielectric constant of the $\mathrm{Al}_{2} \mathrm{O}_{3}$ composite. A visual analysis of the accuracy of the equation can be quickly inferred using Figure 4.12 and quantified using Table 4.12. Leading swiftly to the conclusion that the series mixing rule does not work for this constitutive powder. The average percent error of the Series mixing equation across all volume loadings was found to be $13.90 \%$. This represents an increased error of $12.54 \%$ from the previous sections constitutive using the same equation. At none of the experimental measurement points does the series mixing equation pass within the t-distribution for this dielectric inclusion ratio. The series mixing rule chronically under predicts the dielectric constant of the compound within the volume loading studied.

Table 4.12: Absolute Percent Error for $\mathrm{Al}_{2} \mathrm{O}_{3}$ composites using the Series Mixing equation.

\begin{tabular}{|l|l|l|l|l|}
\hline $\mathrm{Al}_{2} \mathbf{O}_{3}$ & $\mathbf{5 \%}$ VL & $\mathbf{1 0 \%}$ VL & $\mathbf{2 0 \%}$ VL & $\mathbf{3 0 \%}$ VL \\
\hline Percent Error & $7.91 \%$ & $16.02 \%$ & $16.72 \%$ & $14.94 \%$ \\
\hline
\end{tabular}

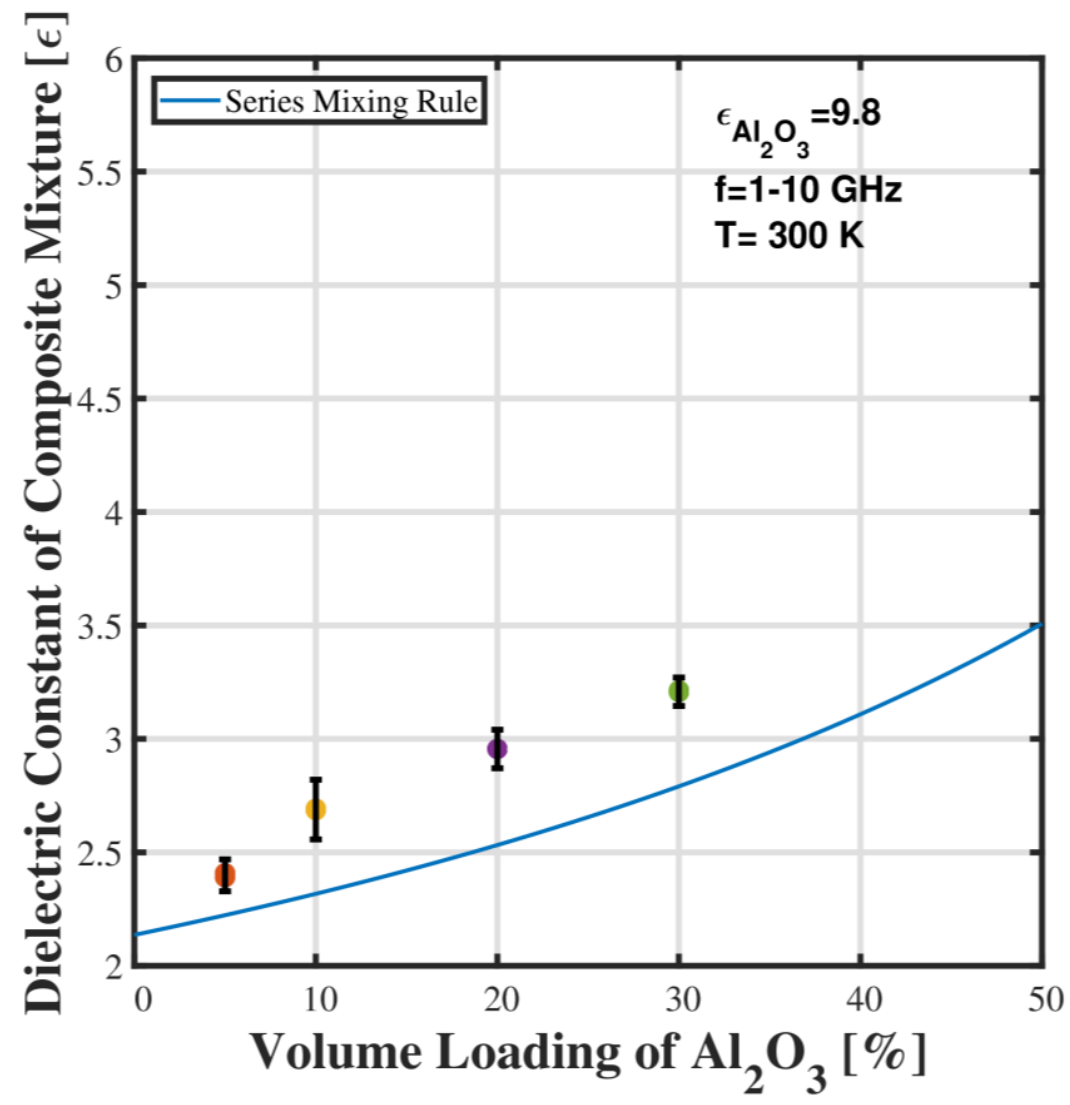

Figure 4.12: Theoretical Series mixture equation for $\mathrm{Al}_{2} \mathrm{O}_{3}$ mapped against experimental scatter plot data for four different volume loadings. 


\subsubsection{Theoretical Logarithmic Mixing Equation Results for $\mathrm{Al}_{2} \mathrm{O}_{3}$}

The Logarithmic Mixing equation is also evaluated for its ability to accurately predict the dielectric constant of $\mathrm{Al}_{2} \mathrm{O}_{3}$ composite's. The graphical information in Figure 4.13 clearly shows that the logarithmic mixture equation does not at any point pass within the t-distribution of the scatter plot. It does not follow the general pattern of the increasing dialectic constant for this composite at any of the measured points. An analysis of Table 4.13 shows that the percent error is an average of $16.95 \%$. This is actually an increase in overall accuracy of $9.02 \%$ from the previous section. The logarithmic equation in this application overpredicts the compounds dielectric equation at each point, clearly indicating that this equation is not valid for this inclusion ratio.

Table 4.13: Absolute Percent Error for $\mathrm{Al}_{2} \mathrm{O}_{3}$ composites using the Logarithmic Mixing equation.

\begin{tabular}{|l|l|l|l|l|}
\hline $\mathrm{Al}_{2} \mathrm{O}_{3}$ & $\mathbf{5 \%}$ VL & $\mathbf{1 0 \%}$ VL & $\mathbf{2 0 \%}$ VL & $\mathbf{3 0 \%}$ VL \\
\hline Percent Error & $24.50 \%$ & $16.93 \%$ & $13.42 \%$ & $12.94 \%$ \\
\hline
\end{tabular}

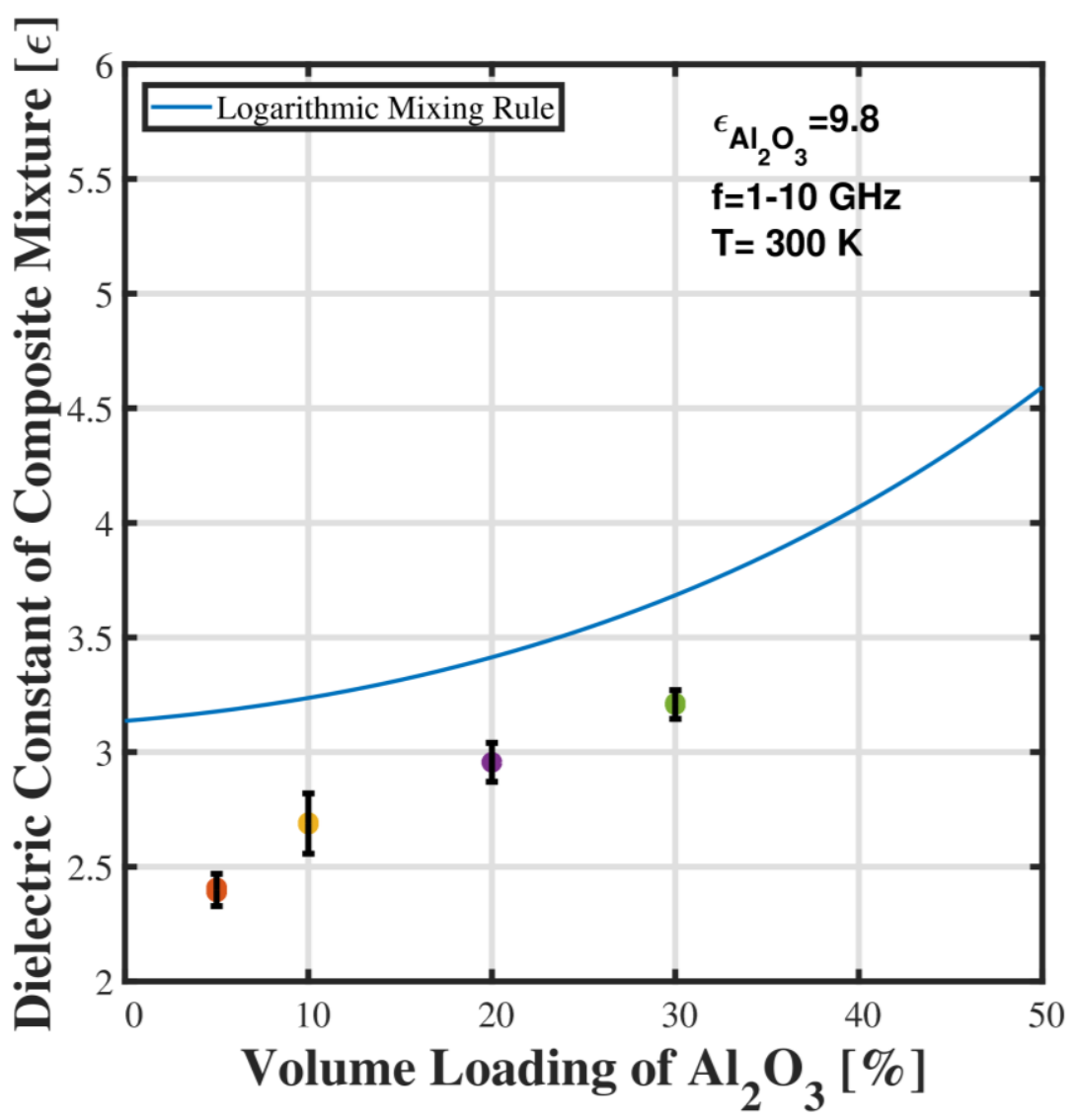

Figure 4.13: Theoretical Logarithmic mixture equation for $\mathrm{Al}_{2} \mathrm{O}_{3}$ mapped against experimental scatter plot data for four different volume loadings. 


\subsubsection{Theoretical Lichtenecker Mixing Equation for $\mathrm{Al}_{2} \mathrm{O}_{3}$}

Continuing the evaluation of $\mathrm{Al}_{2} \mathrm{O}_{3}$ two-phase homogenous composites, the Lichtenecker mixing equation is assessed. Like most of the other equations in this section, the Lichtenecker mixing equation struggles to map the behavior of the changes in dielectric constant as a function of volume loading. It is still relatively accurate despite predicting a higher dielectric constant rate of change then what is seen experimentally. The Lichtenecker equation has an average percent error of $4.77 \%$ an increase of only $3.99 \%$. Figure 4.14 shows that despite this good accuracy $(<5 \%)$ the Lichtenecker mixing equation only passes through the t-distribution at the $20 \%$ volume loading. This continues to support the idea that increasing the dielectric inclusion ratio leads to more difficulty in predicting the behavior of the composite.

Table 4.14: Absolute Percent Error for $\mathrm{Al}_{2} \mathrm{O}_{3}$ composites using the Lichtenecker Mixing equation.

\begin{tabular}{|l|l|l|l|l|}
\hline $\mathrm{Al}_{2} \mathrm{O}_{3}$ & $\mathbf{5 \%}$ VL & $\mathbf{1 0 \%}$ VL & $\mathbf{2 0 \%}$ VL & 30\% VL \\
\hline Percent Error & $4.07 \%$ & $8.08 \%$ & $2.02 \%$ & $4.92 \%$ \\
\hline
\end{tabular}

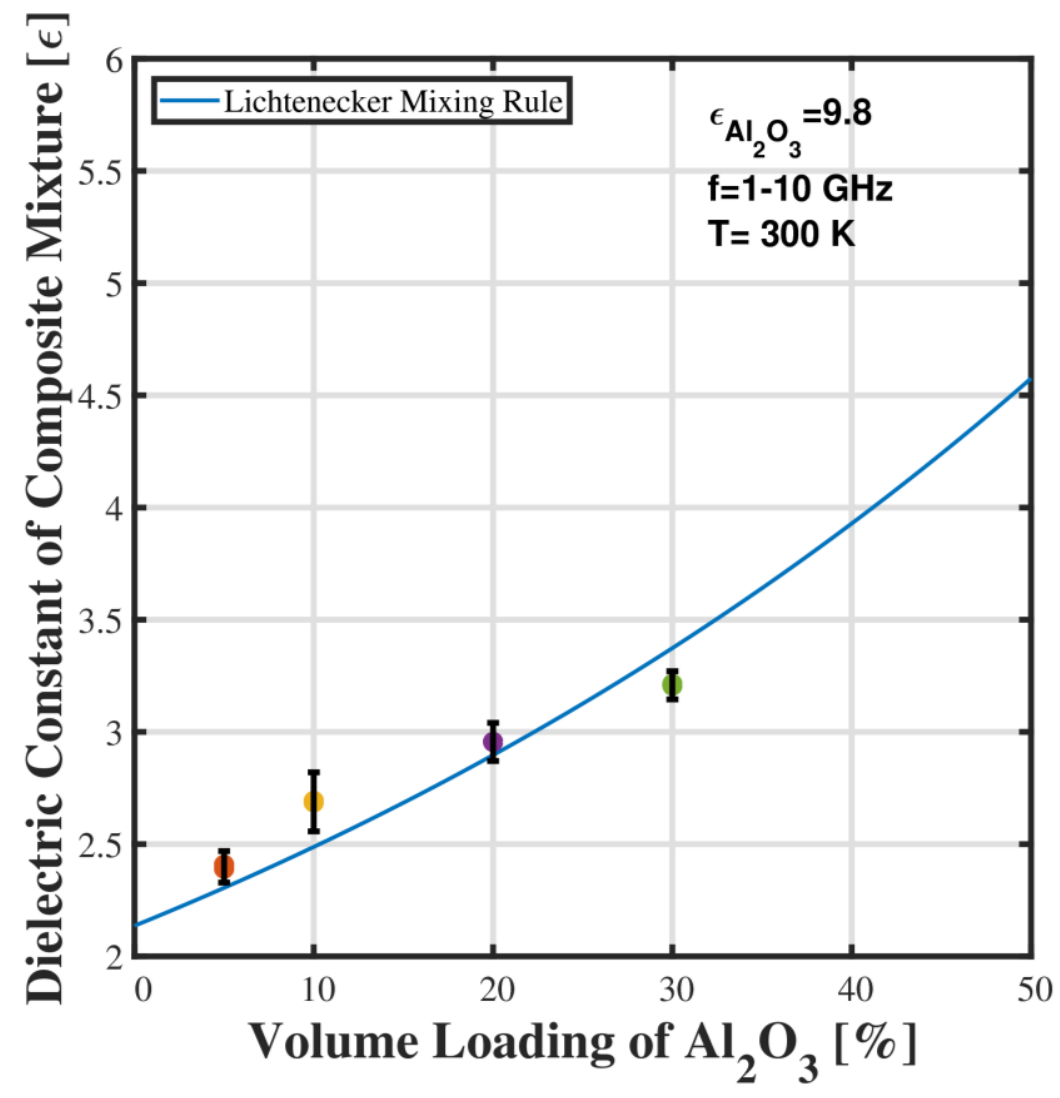

Figure 4.14: Theoretical Lichtenecker mixture equation for $\mathrm{Al}_{2} \mathrm{O}_{3}$ mapped against experimental scatter plot data for four different volume loadings. 


\subsubsection{Theoretical Looyenga Mixing Equation for $\mathrm{Al}_{2} \mathrm{O}_{3}$}

The ensuing equation examined is the series mixing equation for its accuracy in predicting the dielectric constant of the $\mathrm{Al}_{2} \mathrm{O}_{3}$ composite. A visual examination of the accuracy of the equation can be quickly construed using Figure 4.15. This analysis can be quantified using Table 4.15 showing that the average percent error of the Looyenga mixing equation across all volume loadings is $5.79 \%$. The equation is particular accurate at low volume loading $(<20 \%)$, as the volume loading increases though, the accuracy of the equation starts to deteriorate. Using the Looyenga mixing rule for a dielectric inclusion ratio of 4.3 resulted in an average increase in error of $4.82 \%$ from that of the previous sections dielectric inclusion ratio.

Table 4.15: Absolute Percent Error for $\mathrm{Al}_{2} \mathrm{O}_{3}$ composites using the Looyenga Mixing equation.

\begin{tabular}{|l|l|l|l|l|}
\hline $\mathrm{Al}_{2} \mathrm{O}_{3}$ & $\mathbf{5 \%}$ VL & $\mathbf{1 0 \%}$ VL & $\mathbf{2 0 \%}$ VL & $\mathbf{3 0 \%}$ VL \\
\hline Percent Error & $1.86 \%$ & $3.85 \%$ & $4.70 \%$ & $12.77 \%$ \\
\hline
\end{tabular}

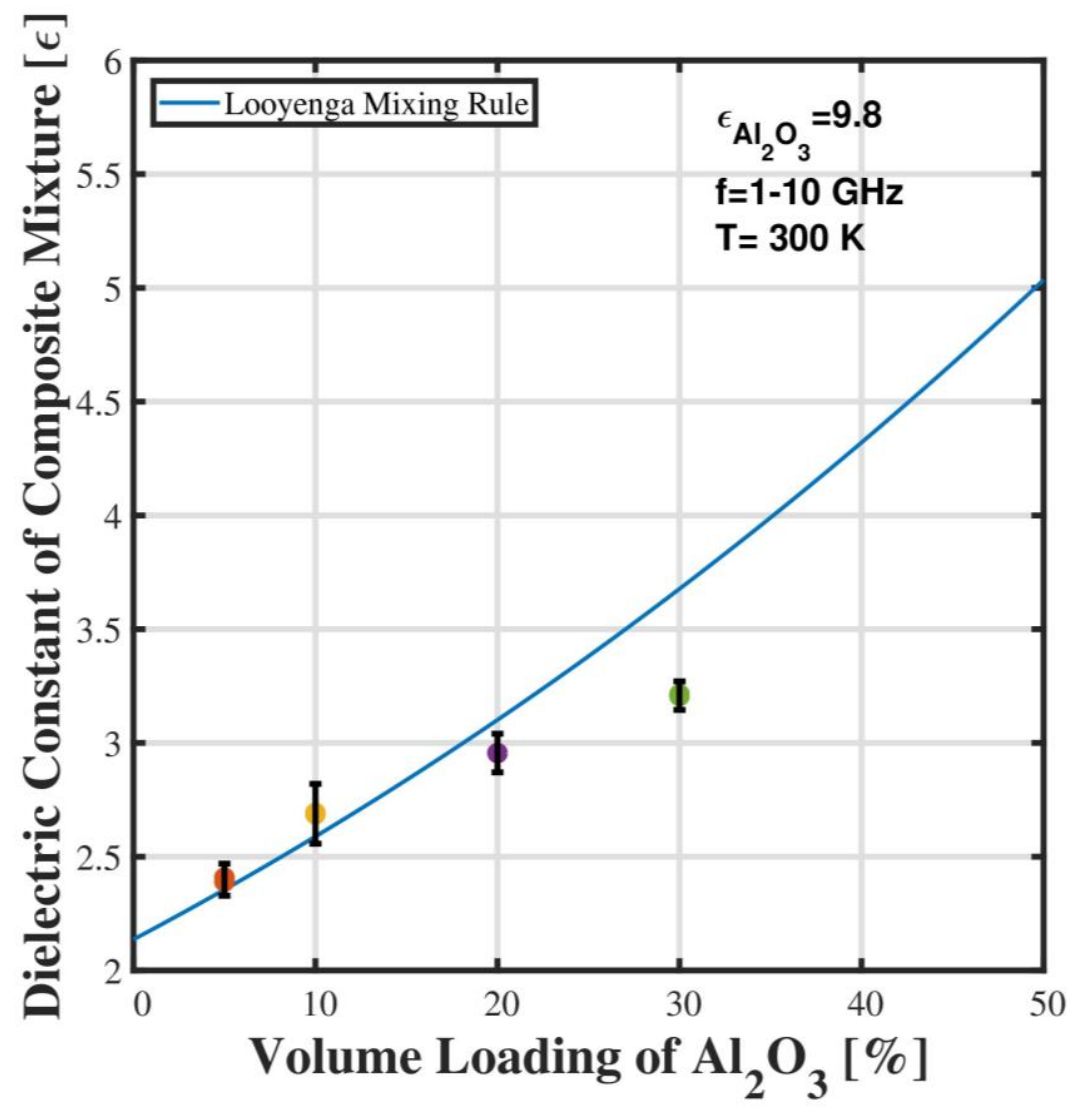

Figure 4.15: Theoretical Looyenga mixture equation for $\mathrm{Al}_{2} \mathrm{O}_{3}$ mapped against experimental scatter plot data for four different volume loadings. 


\subsubsection{Theoretical Birchak Mixing Equation for $\mathrm{Al}_{2} \mathrm{O}_{3}$}

The Birchak equation is used to study a $\mathrm{Al}_{2} \mathrm{O}_{3}$ two-phase homogenous composite increasing volume loadings. Like the Looyenga mixing equation (4.2.5) the Birchak equation is very accurate at low volume loadings, but the accuracy quickly wanes as volume loading increases. The Birchak equation has an average percent error of $6.72 \%$ an increase of $5.65 \%$ from the previous section. Once again this can be easily seen from Figure 4.16, where the Birchak mixing never varies outside of the t-distributions for low volume loadings. This shows that the rate of change of the dielectric constant as volume loading increases is not mathematically characterized for higher volume loadings. Indicating that this mixture equation would only be useful at low volume loadings.

Table 4.16: Absolute Percent Error for $\mathrm{Al}_{2} \mathrm{O}_{3}$ composites using the Birchak Mixing equation.

\begin{tabular}{|l|l|l|l|l|}
\hline $\mathrm{Al}_{2} \mathrm{O}_{3}$ & $\mathbf{5 \%}$ VL & $\mathbf{1 0 \%}$ VL & $\mathbf{2 0 \%}$ VL & $\mathbf{3 0 \%}$ VL \\
\hline Percent Error & $0.50 \%$ & $1.38 \%$ & $8.30 \%$ & $17.69 \%$ \\
\hline
\end{tabular}

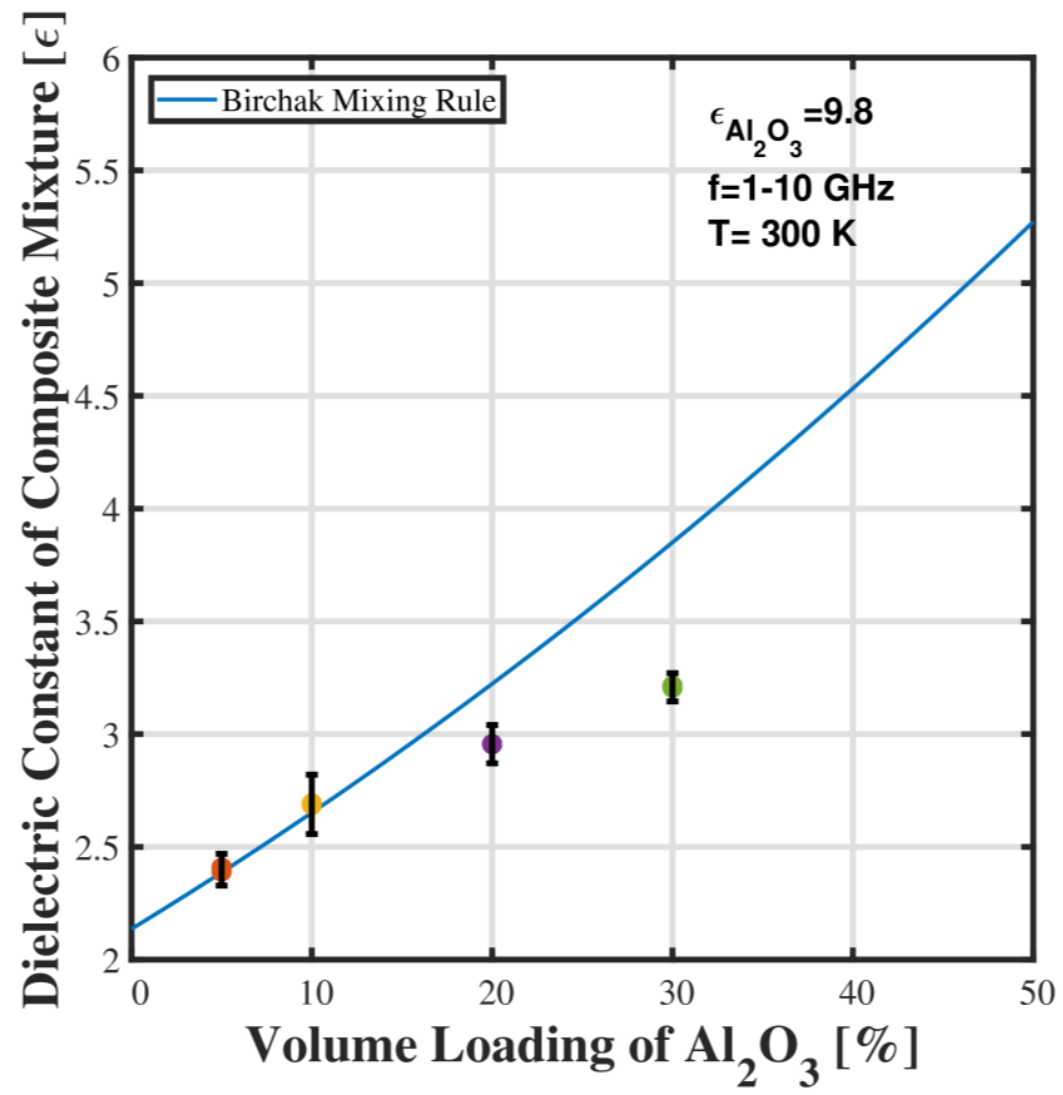

Figure 4.16: Theoretical Birchak mixture equation for $\mathrm{Al}_{2} \mathrm{O}_{3}$ mapped against experimental scatter plot data for four different volume loadings. 


\subsubsection{Theoretical Poon-Shin Mixing Equation for $\mathrm{Al}_{2} \mathrm{O}_{3}$}

Using the Poon-Shin Mixing equation experimental results of the previously discussed $\mathrm{Al}_{2} \mathrm{O}_{3}$-paraffin composites are studied. Like the other equations used for predictive analysis of this dielectric inclusion ratio, the Poon-Shin mixing equation is not extremely accurate. From Table 4.7 it can be seen that the mixture equation's accuracy is good at 5 and 20 percent but poor at 10 and 30 percent, with an average percent error of $5.50 \%$, which is an increase of $4.49 \%$ from the same analysis with $\mathrm{SiO}_{2}$. An analysis of Figure 4.17 shows that this is another equation that does a decent job of mapping the growth of the dielectric constant. While some of the other equation discussed in this study are only good at lower volume loadings, the Poon-Shin equation comparatively predicts slightly lesser values for the dielectric constant at low volume loading and slightly greater values at higher volume loadings.

Table 4.17: Absolute Percent Error for $\mathrm{Al}_{2} \mathrm{O}_{3}$ composites using the Poon-Shin Mixing equation.

\begin{tabular}{|l|l|l|l|l|}
\hline $\mathrm{Al}_{2} \mathbf{O}_{3}$ & $\mathbf{5 \%}$ VL & $\mathbf{1 0 \%}$ VL & $\mathbf{2 0 \%}$ VL & $\mathbf{3 0 \%}$ VL \\
\hline Percent Error & $3.39 \%$ & $6.46 \%$ & $1.62 \%$ & $10.54 \%$ \\
\hline
\end{tabular}

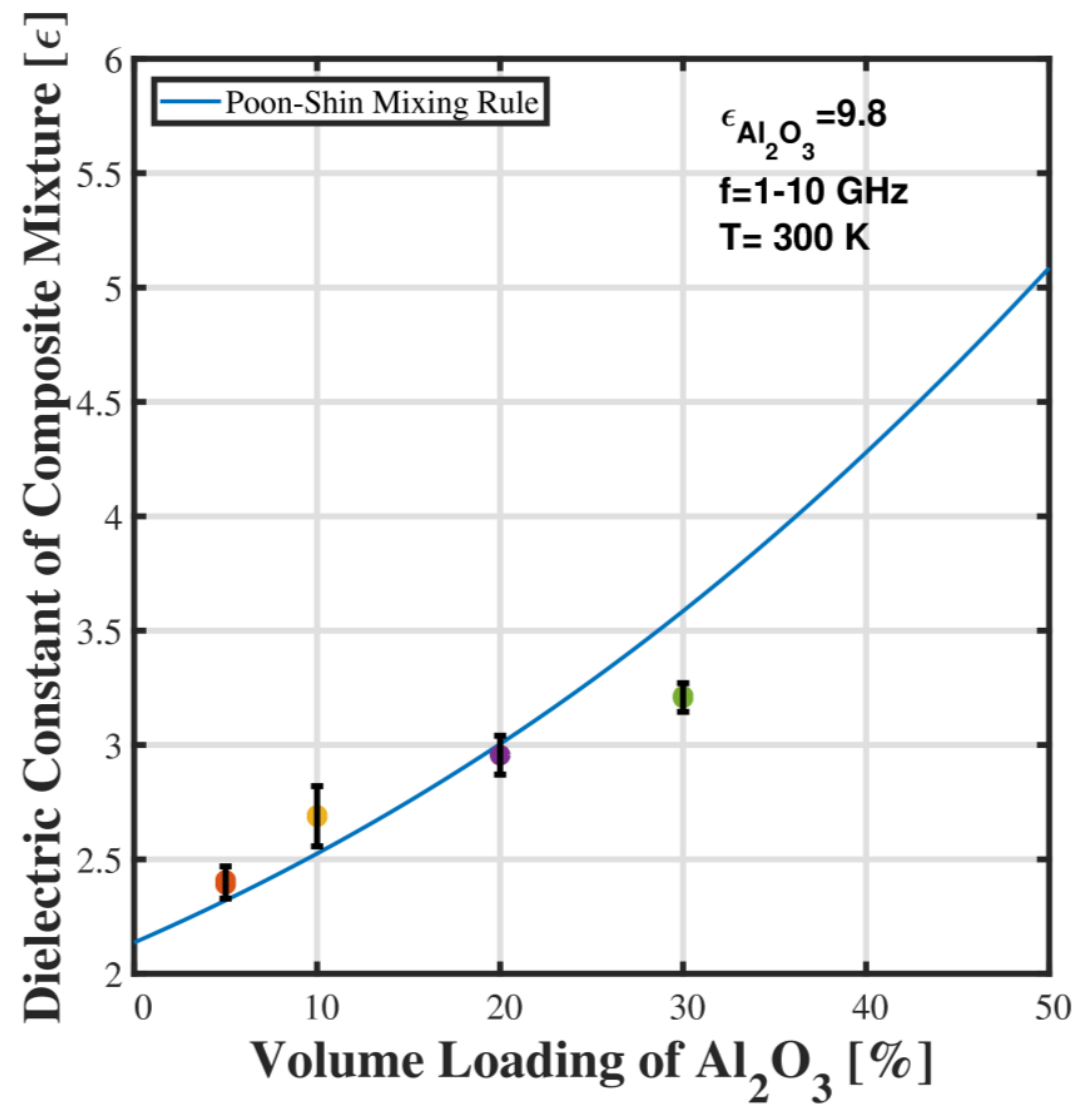

Figure 4.17: Theoretical Poon-Shin mixture equation for $\mathrm{Al}_{2} \mathrm{O}_{3}$ mapped against experimental scatter plot data for four different volume loadings. 


\subsubsection{Theoretical Effective Medium Theory Mixing Equation for $\mathrm{Al}_{2} \mathrm{O}_{3}$}

The EMT mixing equation is evaluated for its ability to accurately predict the dielectric constant of a $\mathrm{Al}_{2} \mathrm{O}_{3}$ composite as a function of volume loading. Unlike the other equations the EMT's shape factor variable allows it to take unique mathematical rates of change, with slower rates of change being expressed at higher volume loadings. The graph in Figure 4.18, reveals that the EMT mixing equation passes within the t-distribution for all volume loadings shown. This is a significant change from the other equations shown in this section. A comparison of Table 4.14 shows that the percent error stays consistently low for all volume loading, with an average percent error of $1.16 \%$. This low error percentage indicates that the equation can accurately follow the increasing trend of this compound within the parameters studied.

Table 4.18: Absolute Percent Error for $\mathrm{Al}_{2} \mathrm{O}_{3}$ composites using the EMT Mixing equation.

\begin{tabular}{|l|l|l|l|l|}
\hline $\mathrm{Al}_{2} \mathbf{O}_{3}$ & $\mathbf{5 \%}$ VL & $\mathbf{1 0 \%}$ VL & $\mathbf{2 0 \%}$ VL & 30\% VL \\
\hline Percent Error & $2.43 \%$ & $0.16 \%$ & $1.82 \%$ & $0.24 \%$ \\
\hline
\end{tabular}

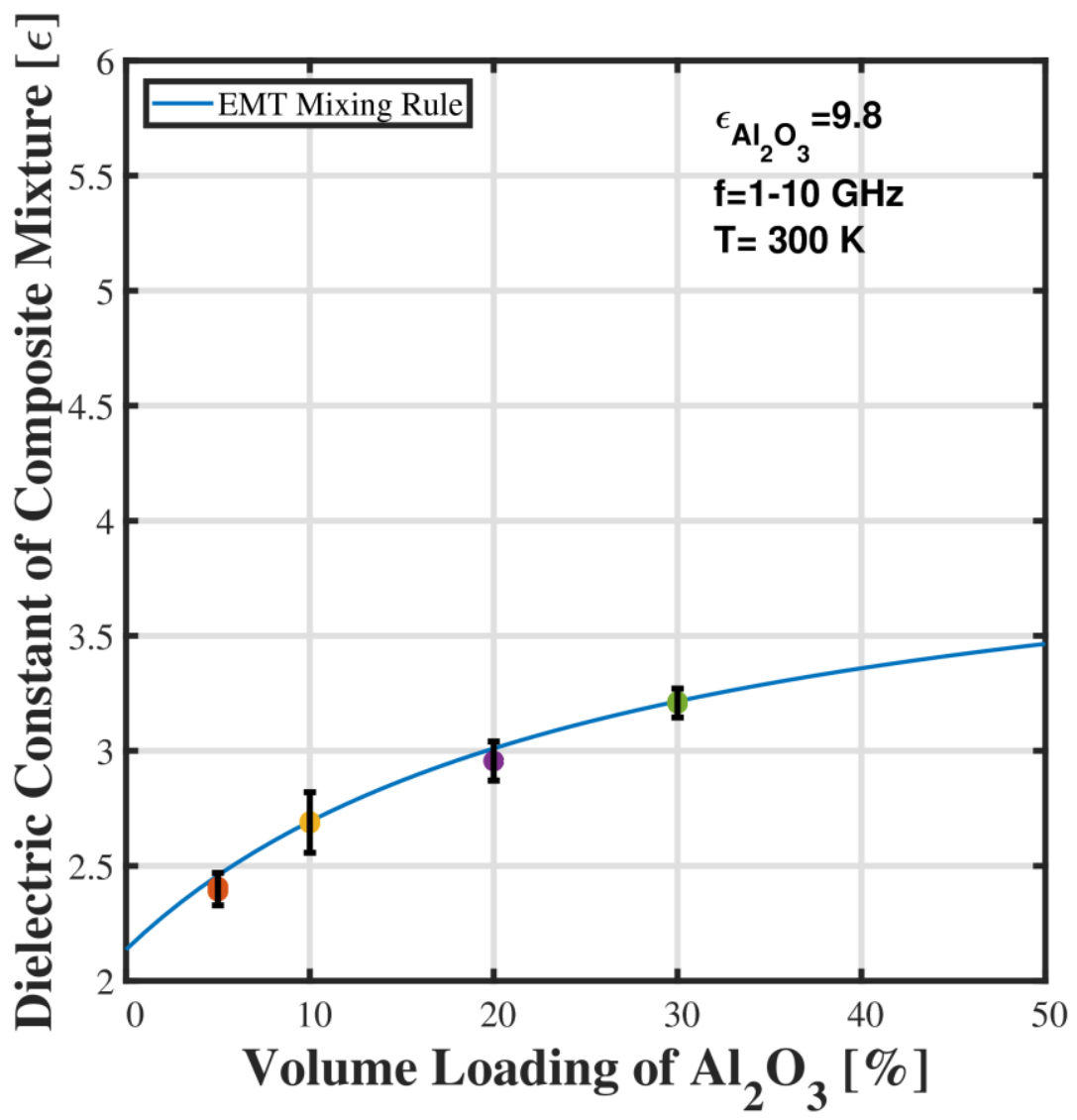

Figure 4.18: Theoretical EMT mixture equation for $\mathrm{Al}_{2} \mathrm{O}_{3}$ mapped against experimental scatter plot data for four different volume loadings. 


\subsubsection{Theoretical Maxwell-Garnet Mixing Equation for $\mathrm{Al}_{2} \mathrm{O}_{3}$}

The Maxwell-Garnet Mixing equation is the next equation evaluated in this section. It can be inferred from the graphical information in Figure 4.19 that the Maxwell-Garnet Mixture equation does a respectable job of predicting the dielectric constant of the mixture as the equation passes within two of the error bars on the scatter plot. A comparison with Table 4.19 shows quantitively that at each of the volume loading where the equation passes within the error bars the Maxwell-Garnet Mixing equation calculates the error to within $5 \%$. The average percent error across all volume loadings for the Maxwell-Garnet Mixing equation is $4.37 \%$ which is relatively low in comparison to the other equations for this powder loading. This represents an increase of $3.4 \%$ over the previous constitutive powder examined.

Table 4.19: Absolute Percent Error for $\mathrm{Al}_{2} \mathrm{O}_{3}$ composites using the Maxwell-Garnet Mixing equation.

\begin{tabular}{|l|l|l|l|l|}
\hline $\mathrm{Al}_{2} \mathbf{O}_{3}$ & $\mathbf{5 \%}$ VL & $\mathbf{1 0 \%}$ VL & $\mathbf{2 0 \%}$ VL & $\mathbf{3 0 \%}$ VL \\
\hline Percent Error & $3.60 \%$ & $7.32 \%$ & $1.24 \%$ & $5.31 \%$ \\
\hline
\end{tabular}

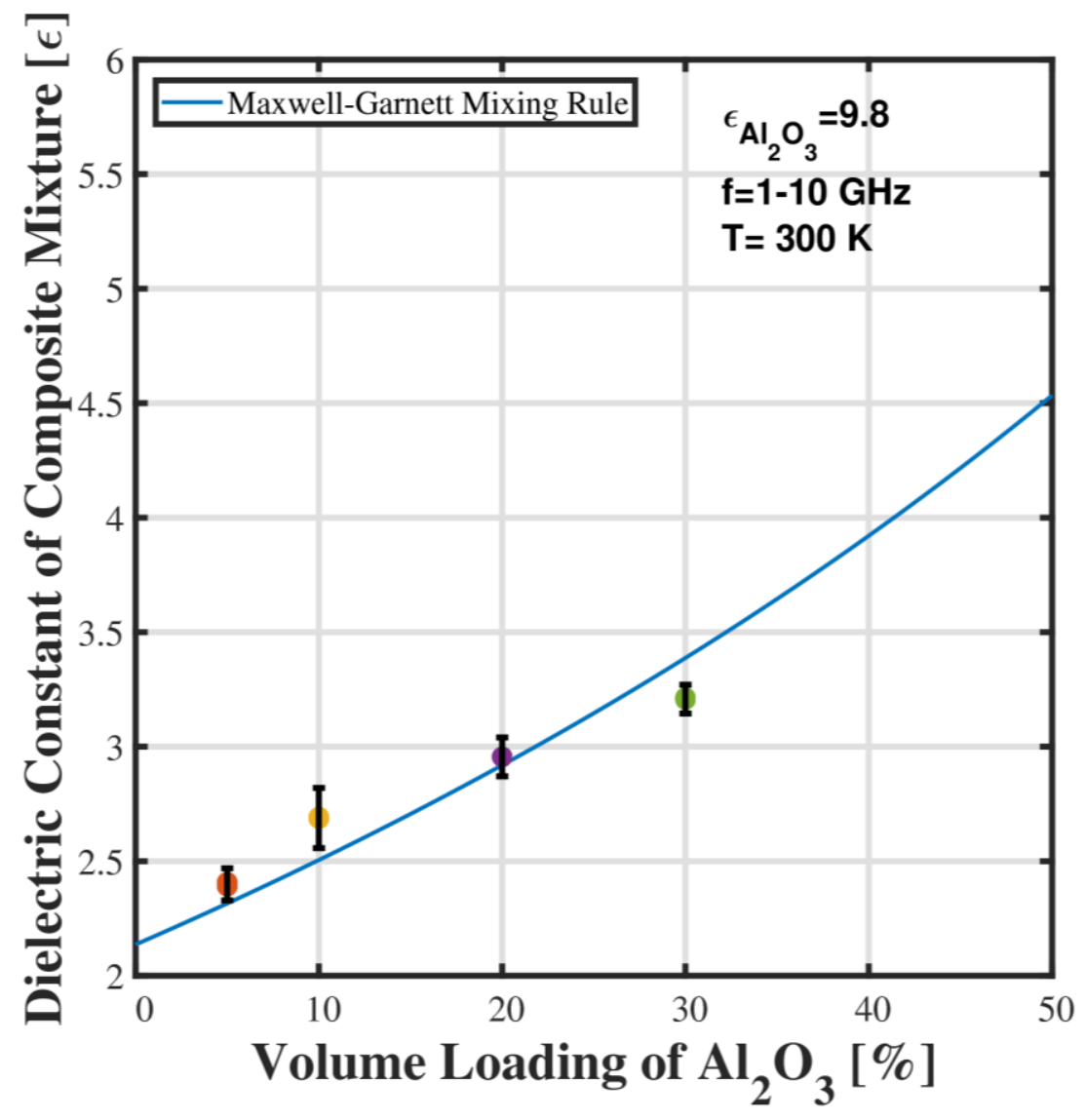

Figure 4.19: Theoretical Maxwell-Garnett mixture equation for $\mathrm{Al}_{2} \mathrm{O}_{3}$ mapped against experimental scatter plot data for four different volume loadings. 


\subsubsection{Theoretical Jayasundere-Smith Mixing Equation for $\mathrm{Al}_{2} \mathrm{O}_{3}$}

The final equation analyzed was the Jayasundere-Smith mixing equation. Table 4.20 shows that the accuracy of the Looyenga mixing equation like many of the others in this section show a worsening accuracy as volume loading increases. With an overall error of $7.70 \%$ across all volume loadings an increase of $6.52 \%$ from the previous section, these results can be clearly clarified by a comparison with Figure 4.20. The mixture equation once again passes through the t-distribution for the low volume loading compounds $(<20 \%)$. Providing an indicator that this might be another equation that is suitable for low dielectric inclusion ratios. However, at high volume loadings (>20\%) the equation is widely outside the t-distribution, indicating that this equation might not be suitable for high volume loadings.

Table 4.20: Absolute Percent Error for $\mathrm{Al}_{2} \mathrm{O}_{3}$ composites using the Jayasundere-Smith Mixing equation.

\begin{tabular}{|l|l|l|l|l|}
\hline $\mathrm{Al}_{2} \mathbf{O}_{3}$ & $\mathbf{5 \%}$ VL & $\mathbf{1 0 \%}$ VL & $\mathbf{2 0 \%}$ VL & 30\% VL \\
\hline Percent Error & $2.97 \%$ & $4.93 \%$ & $5.93 \%$ & $16.96 \%$ \\
\hline
\end{tabular}

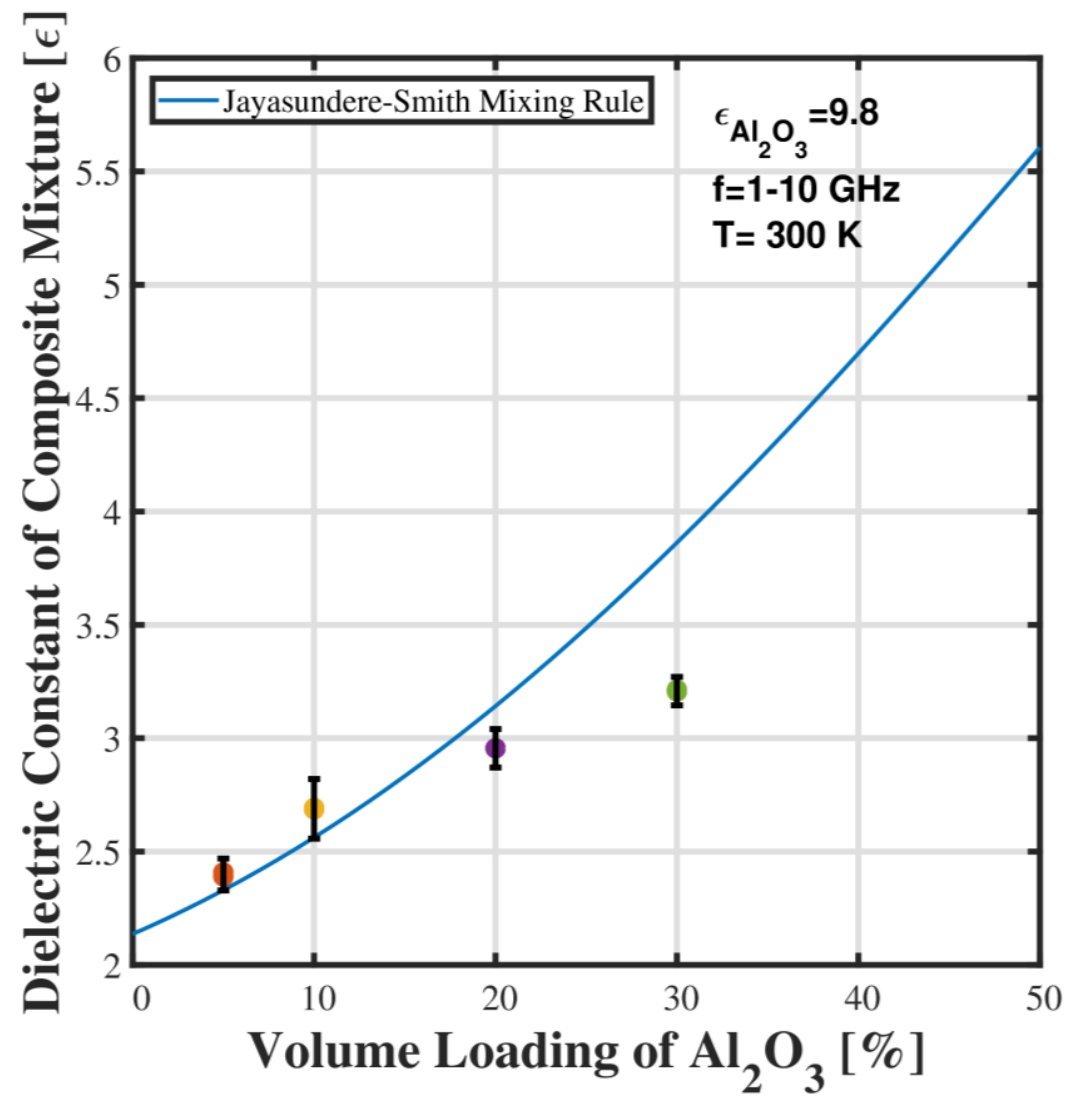

Figure 4.20: Theoretical Jayasundere-Smith mixture equation for $\mathrm{Al}_{2} \mathrm{O}_{3}$ mapped against experimental scatter plot data for four different volume loadings. 


\subsection{Cerium Dioxide Composite Testing Results}

The study next looked at a $\mathrm{CeO}_{2}$ as the inclusion powder to ascertain the accuracy of the mixture equations for this work. The higher dielectric constant of $\mathrm{CeO}_{2}\left(\varepsilon_{p}=21.3\right)$ results in a dielectric inclusion ratio of $\varepsilon_{p} / \varepsilon_{m}=10$. The theoretical outcomes of the mixture equations as a function of volume loading are graphed against the experimental scatter plot data in Figures 4.214.30 for $\mathrm{CeO}_{2}$. The absolute percent error between the different measured $\mathrm{CeO}_{2}$ composite and the theoretical output of each mixture equation are represented in Tables 4.21-4.30.

\subsubsection{Theoretical Parallel Mixing Equation Results for $\mathrm{CeO}_{2}$}

Like the previous section the first equation evaluated in the Parallel Mixing equation. The Parallel Mixing equation has an average percent error across all volume loadings of $27.99 \%$. This is an increase of $13.77 \%$ error from the same equation using $\mathrm{Al}_{2} \mathrm{O}_{3}$ as the constitutive material. Figure 4.21 and Table 4.21 shows that the parallel mixing rule does a terrible job of predicting the behavior of the composites dielectric constant as volume loading increases just like for the previously studied powder. It can be seen from the Figure that the behavior of the measured composite's dielectric constant behaves exponentially whilst the parallel mixture equation only predicts a linear growth. The effects of the increased dielectric inclusion ratio on the accuracy of the mixture equations can clearly be seen in this section.

Table 4.21: Absolute Percent Error for $\mathrm{CeO}_{2}$ composites using the Parallel Mixing equation.

\begin{tabular}{|l|l|l|l|l|}
\hline $\mathrm{CeO}_{2}$ & $\mathbf{5 \%}$ VL & $\mathbf{1 0 \%}$ VL & $\mathbf{2 0 \%}$ VL & 30\% VL \\
\hline Percent Error & $17.71 \%$ & $27.70 \%$ & $33.01 \%$ & $33.54 \%$ \\
\hline
\end{tabular}




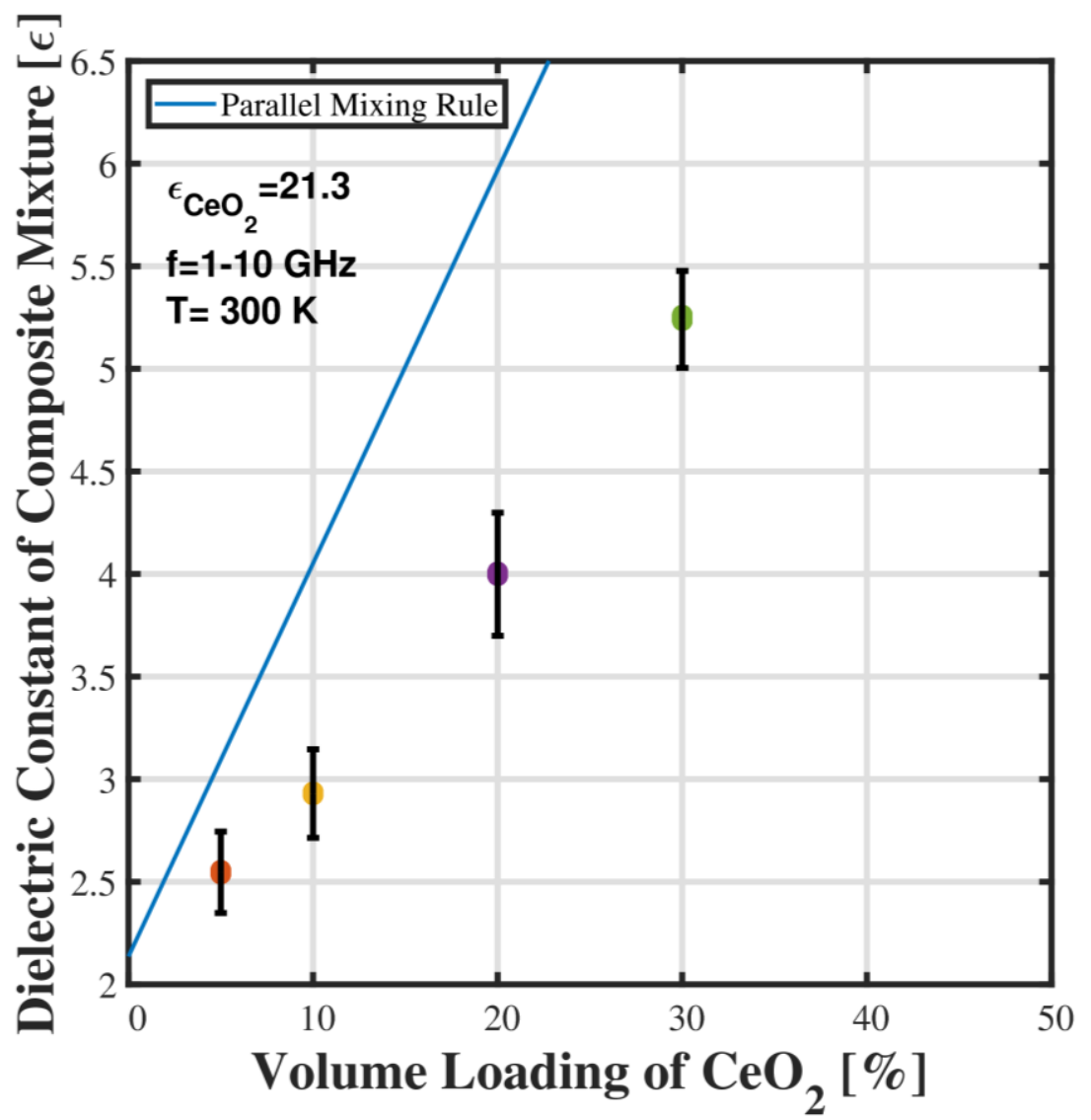

Figure 4.21: Theoretical Parallel mixture equation for $\mathrm{CeO}_{2}$ mapped against experimental scatter plot data for four different volume loadings. 


\subsubsection{Theoretical Series Mixing Equation Results for $\mathrm{CeO}_{2}$}

The next equation investigated is the series mixing equation for its validity in predicting the dielectric constant of the $\mathrm{CeO}_{2}$ composite. A graphic analysis of the equation can be quickly gathered using Figure 4.22 and quantified using Table 4.22. The average percent error of the Series mixing equation across all volume loadings was found to be $42.83 \%$. This is a $28.93 \%$ increase in error then what was seen for the $\mathrm{Al}_{2} \mathrm{O}_{3}$ composites using the same equation. At none of the experimental measurement points does the series mixing equation pass within the t-distribution for this dielectric inclusion ratio. The series mixture equation under predicts the compound's dielectric constant with exponential error correlating to volume loading.

Table 4.22: Absolute Percent Error for $\mathrm{CeO}_{2}$ composites using the Series Mixing equation.

\begin{tabular}{|l|l|l|l|l|}
\hline $\mathrm{CeO}_{2}$ & $\mathbf{5 \%}$ VL & $\mathbf{1 0 \%}$ VL & $\mathbf{2 0 \%}$ VL & 30\% VL \\
\hline Percent Error & $13.85 \%$ & $24.83 \%$ & $53.52 \%$ & $79.12 \%$ \\
\hline
\end{tabular}

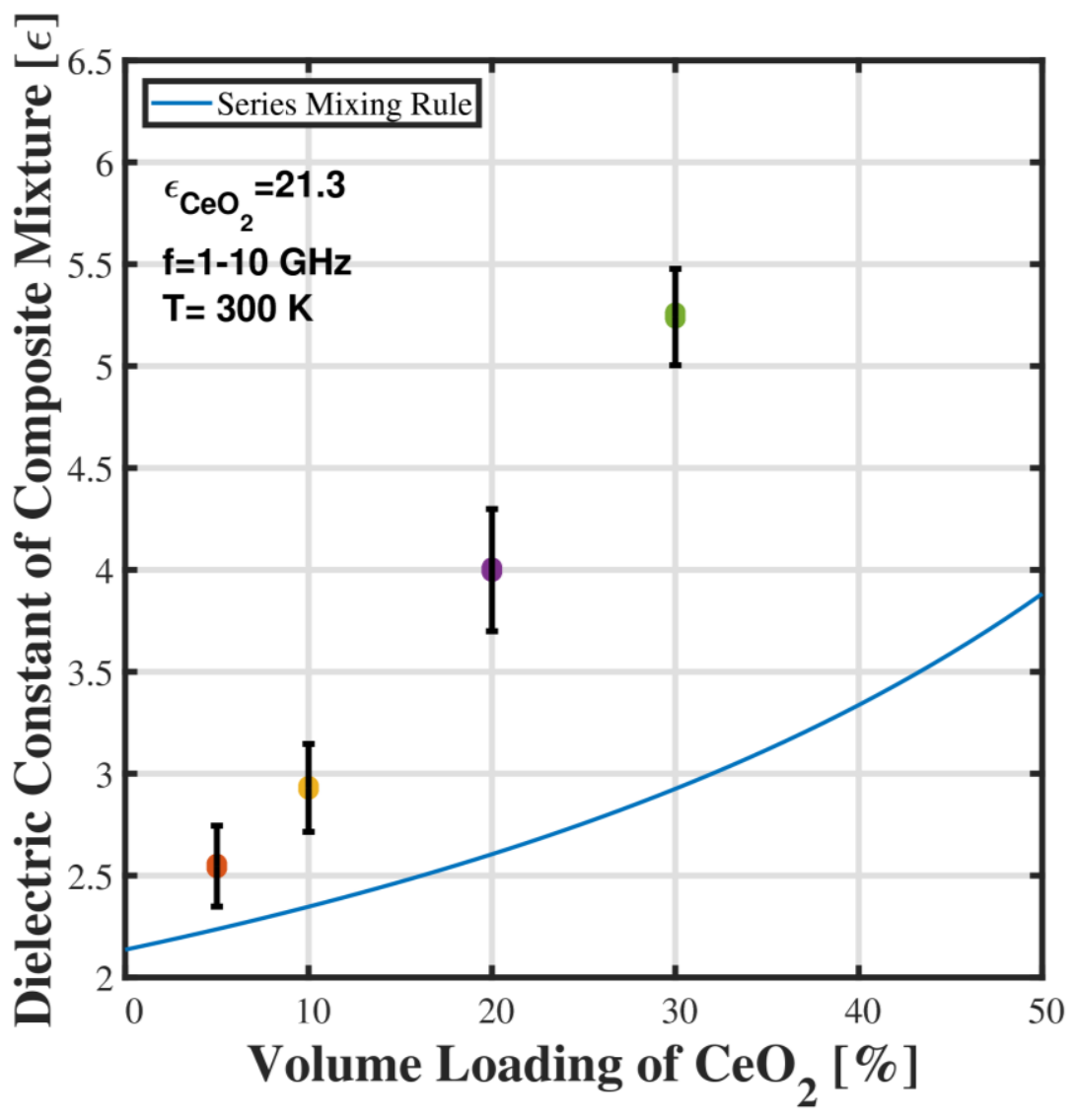

Figure 4.22: Theoretical Series mixture equation for $\mathrm{CeO}_{2}$ mapped against experimental scatter plot data for four different volume loadings. 


\subsubsection{Theoretical Logarithmic Mixing Equation Results for $\mathrm{CeO}_{2}$}

The Logarithmic Mixing equation is also evaluated for its ability to accurately predict the dielectric constant of a $\mathrm{CeO}_{2}$ composite as a function of volume loading. It can be summarized from the graphical information in Figure 4.23 that the logarithmic mixture equation does not at pass within the t-distribution of the scatter plot. It does not come close to following the general pattern of the increasing dielectric constant for this composite. The equation does pass through the distribution at, at least a single point. This can be inferred from the decreasing dielectric constant from 10 to 20 percent volume loading. An analysis of Table 4.23 shows that the average percent error is $16.63 \%$. This is actually an increase in accuracy from the usage of the same equation for $\mathrm{Al}_{2} \mathrm{O}_{3}$ by a factor of $0.32 \%$, while still not accurate it indicates that the power law family of equations might be better suited for this dielectric inclusion ratio.

Table 4.23: Absolute Percent Error for $\mathrm{CeO}_{2}$ composites using the Logarithmic Mixing equation.

\begin{tabular}{|l|l|l|l|l|}
\hline $\mathrm{CeO}_{2}$ & 5\% VL & $\mathbf{1 0 \%}$ VL & $\mathbf{2 0 \%}$ VL & 30\% VL \\
\hline Percent Error & $20.96 \%$ & $12.22 \%$ & $8.70 \%$ & $16.63 \%$ \\
\hline
\end{tabular}

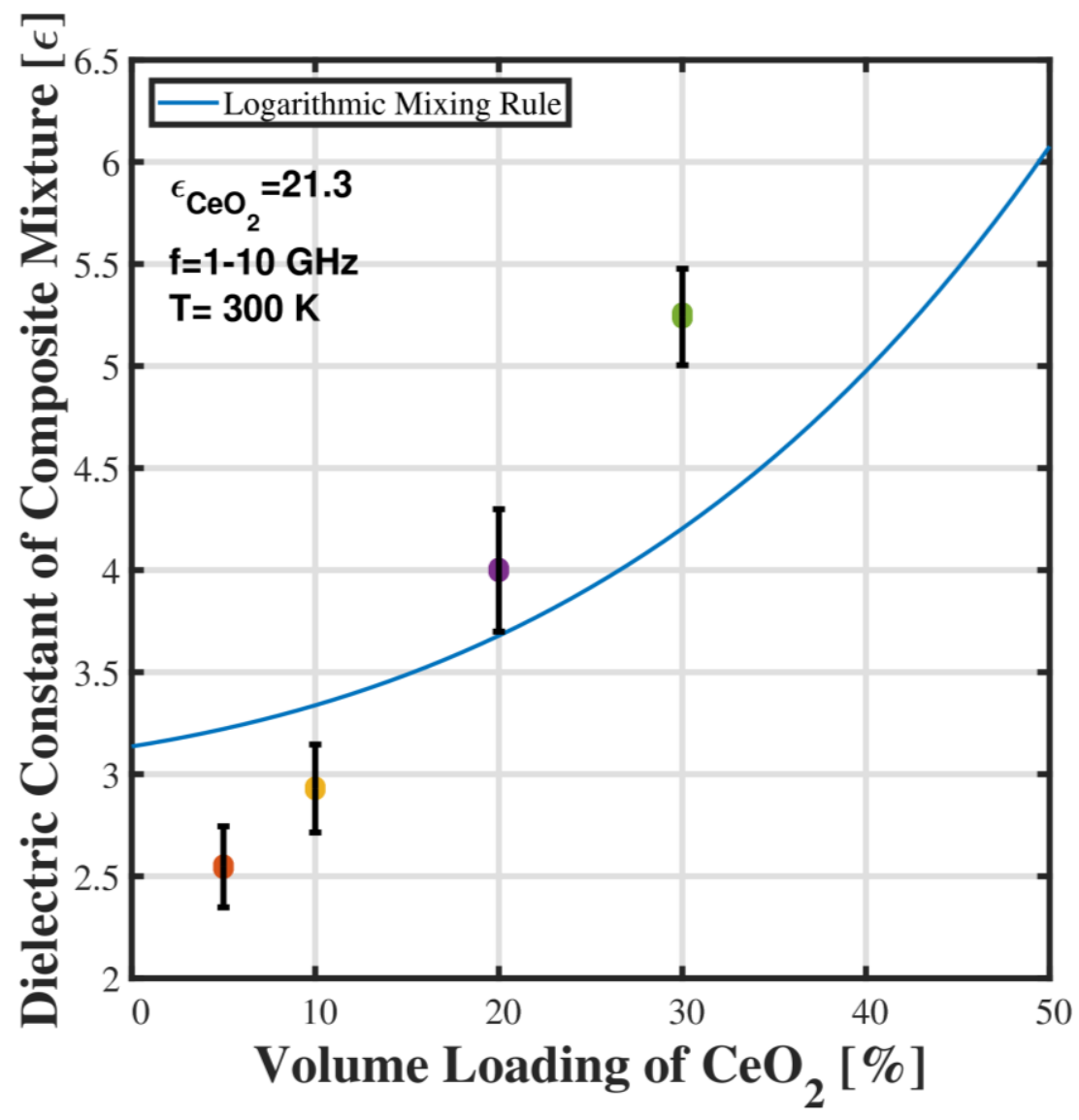

Figure 4.23: Theoretical Logarithmic mixture equation for $\mathrm{CeO}_{2}$ mapped against experimental scatter plot data for four different volume loadings. 


\subsubsection{Theoretical Lichtenecker Mixing Equation for $\mathrm{CeO}_{2}$}

The ensuing equation evaluated for a $\mathrm{CeO}_{2}$ two-phase homogenous composites ability to be mapped using a mixture equation's the Lichtenecker mixing. The Lichtenecker mixing equation is struggles to map the behavior of the changes in dielectric constant as a function of volume loading. It has a relatively accurate exponential growth despite predicting a lower dielectric constant rate of change then what is seen experimentally. The Lichtenecker equation has an average percent error of $14.12 \%$. This is an average accuracy prediction decrease of $9.35 \%$ from the previous $\mathrm{Al}_{2} \mathrm{O}_{3}$ powder using the Lichtenecker mixing equation. Figure 4.24 shows that the Lichtenecker mixing equation only passes through the t-distribution at the 5\% volume loading. An indication that this equation could be used relatively accurately at very low volume loadings.

Table 4.24: Absolute Percent Error for $\mathrm{CeO}_{2}$ composites using the Lichtenecker Mixing equation.

\begin{tabular}{|l|l|l|l|l|}
\hline $\mathrm{CeO}_{2}$ & 5\% VL & $\mathbf{1 0 \%}$ VL & $\mathbf{2 0 \%}$ VL & 30\% VL \\
\hline Percent Error & $6.26 \%$ & $8.99 \%$ & $18.18 \%$ & $23.07 \%$ \\
\hline
\end{tabular}

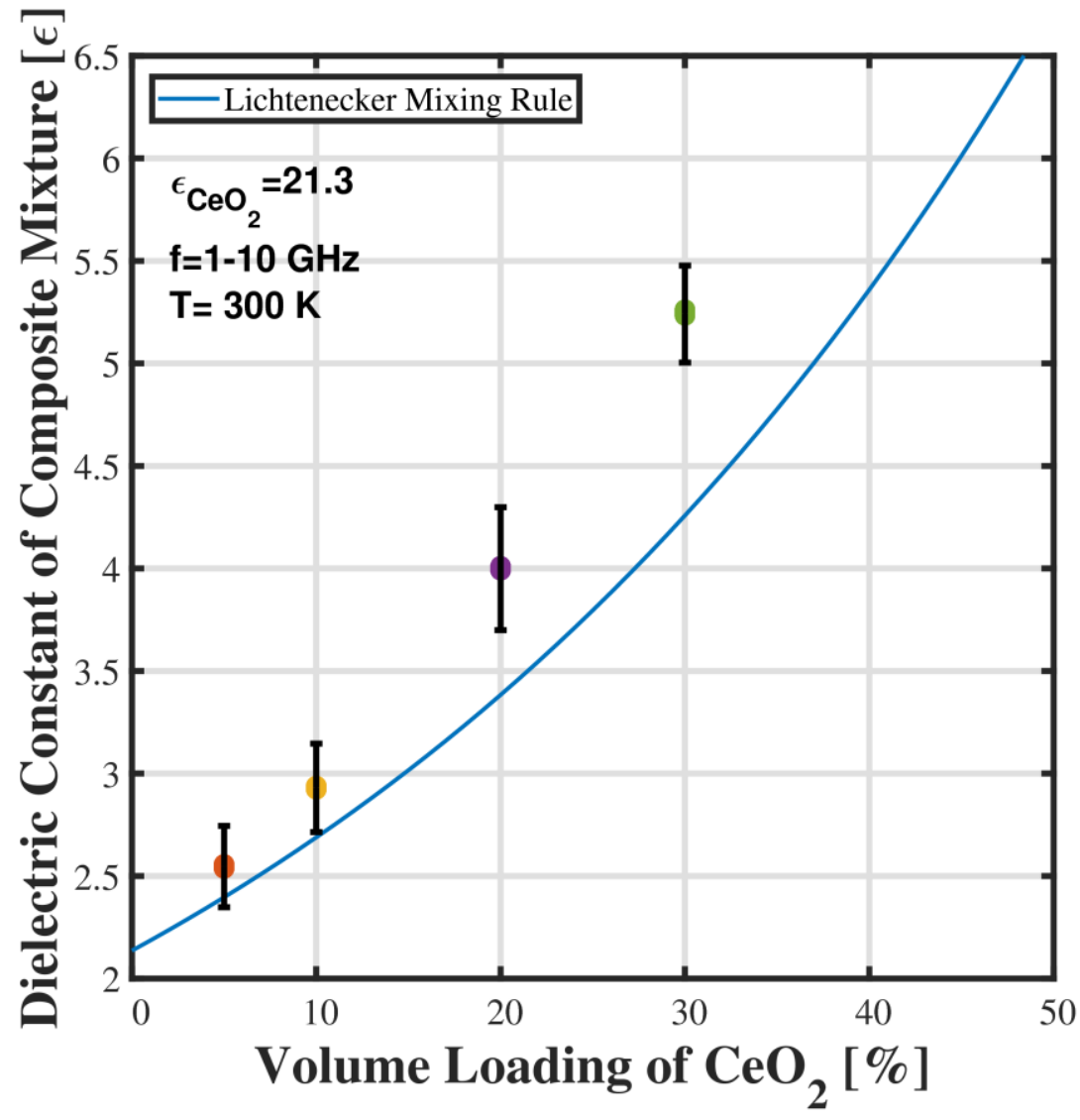

Figure 4.24: Theoretical Lichtenecker mixture equation for $\mathrm{CeO}_{2}$ mapped against experimental scatter plot data for four different volume loadings. 


\subsubsection{Theoretical Looyenga Mixing Equation for $\mathrm{CeO}_{2}$}

The Looyenga rule is next evaluated and compared to the previous sections results. A graphical examination of the mixing equation can be quickly construed via Figure 4.25. The average percent error of the Looyenga mixing equation across all volume loadings was found to be $0.76 \%$. This represents an extremely accurate equation for this application with the theoretical output almost exactly predicting the compound's experimental measurements. This analysis can be quantified using Table 4.25. The usage of the Looyenga mixing rule in predicting the output of the $\mathrm{CeO}_{2}$ compound represents an increase of 5.03 from the previous section. The Looyenga mixing rule is able to exactly match the correlation between volume loading and dielectric constant.

Table 4.25: Absolute Percent Error for $\mathrm{CeO}_{2}$ composites using the Looyenga Mixing equation.

\begin{tabular}{|l|l|l|l|l|}
\hline $\mathrm{CeO}_{2}$ & 5\% VL & $\mathbf{1 0 \%}$ VL & $\mathbf{2 0 \%}$ VL & 30\% VL \\
\hline Percent Error & $0.77 \%$ & $1.11 \%$ & $0.48 \%$ & $0.67 \%$ \\
\hline
\end{tabular}

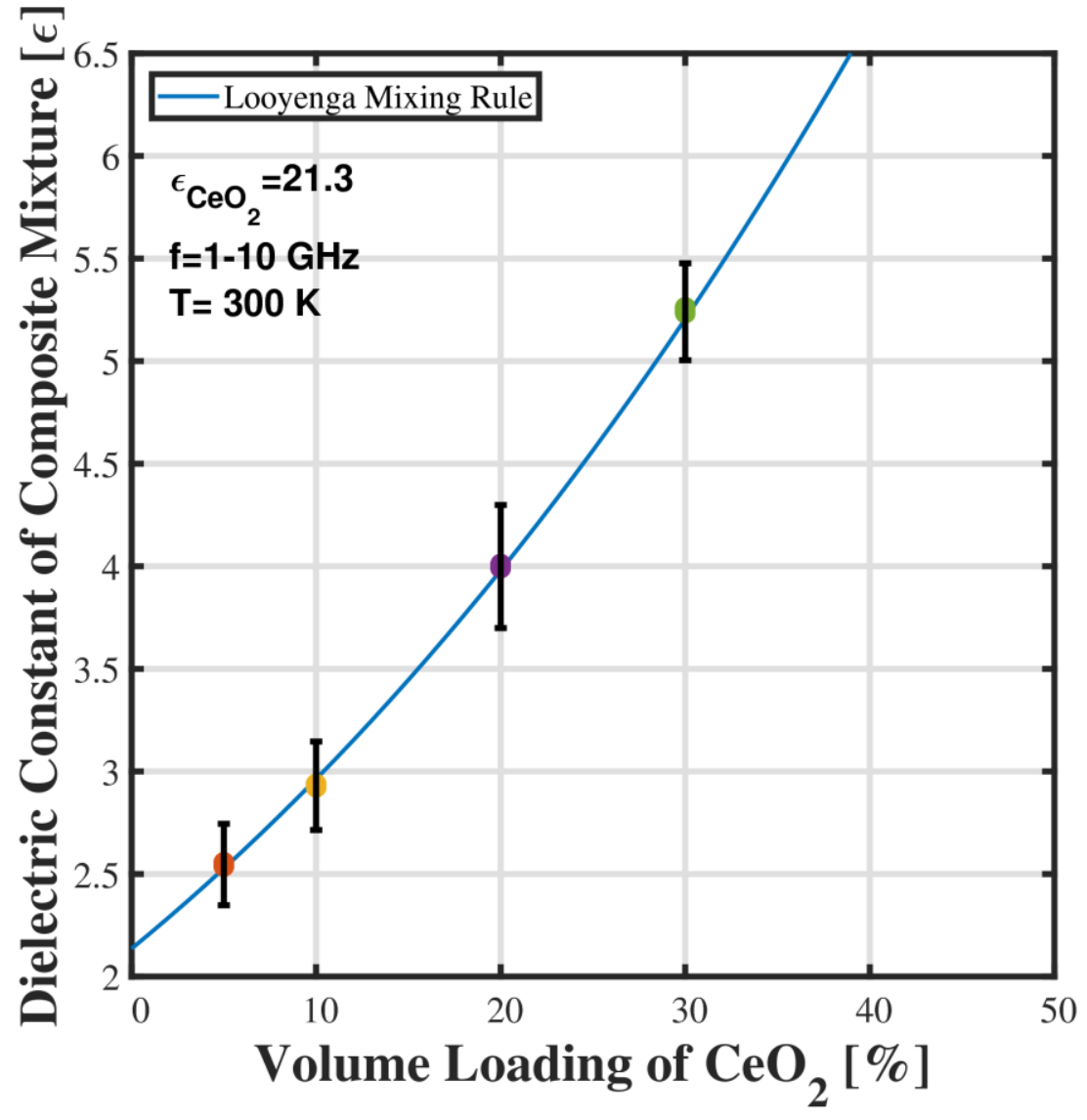

Figure 4.25: Theoretical Looyenga mixture equation for $\mathrm{CeO}_{2}$ mapped against experimental scatter plot data for four different volume loadings. 


\subsubsection{Theoretical Birchak Mixing Equation for $\mathrm{CeO}_{2}$}

Continuing the evaluation of a $\mathrm{CeO}_{2}$ two-phase homogenous compound, the next equation applied is the Birchak mixing equation. Like the Lichtenecker mixing equation (4.3.4) the Birchak equation is very accurate at low volume loadings $(<10 \%)$ but the accuracy quickly wanes as volume loading increases. The Birchak equation has an average percent error of $7.08 \%$. Once again this can be easily seen from Figure 4.26, where the Birchak mixing equation using the ideal dielectric constant of $\mathrm{CeO}_{2}$ varies outside of the t-distributions for all volume loadings greater than $5 \%$. At this higher dielectric inclusion ratio for $\mathrm{CeO}_{2}$ as compared to $\mathrm{Al}_{2} \mathrm{O}_{3}$ the Birchak's mixing equation is slightly less accurate with a decreased average accuracy of $0.62 \%$.

Table 4.26: Absolute Percent Error for $\mathrm{CeO}_{2}$ composites using the Birchak Mixing equation.

\begin{tabular}{|l|l|l|l|l|}
\hline $\mathrm{CeO}_{2}$ & $\mathbf{5 \%}$ VL & $\mathbf{1 0 \%}$ VL & $\mathbf{2 0 \%}$ VL & 30\% VL \\
\hline Percent Error & $2.88 \%$ & $7.20 \%$ & $8.65 \%$ & $9.59 \%$ \\
\hline
\end{tabular}

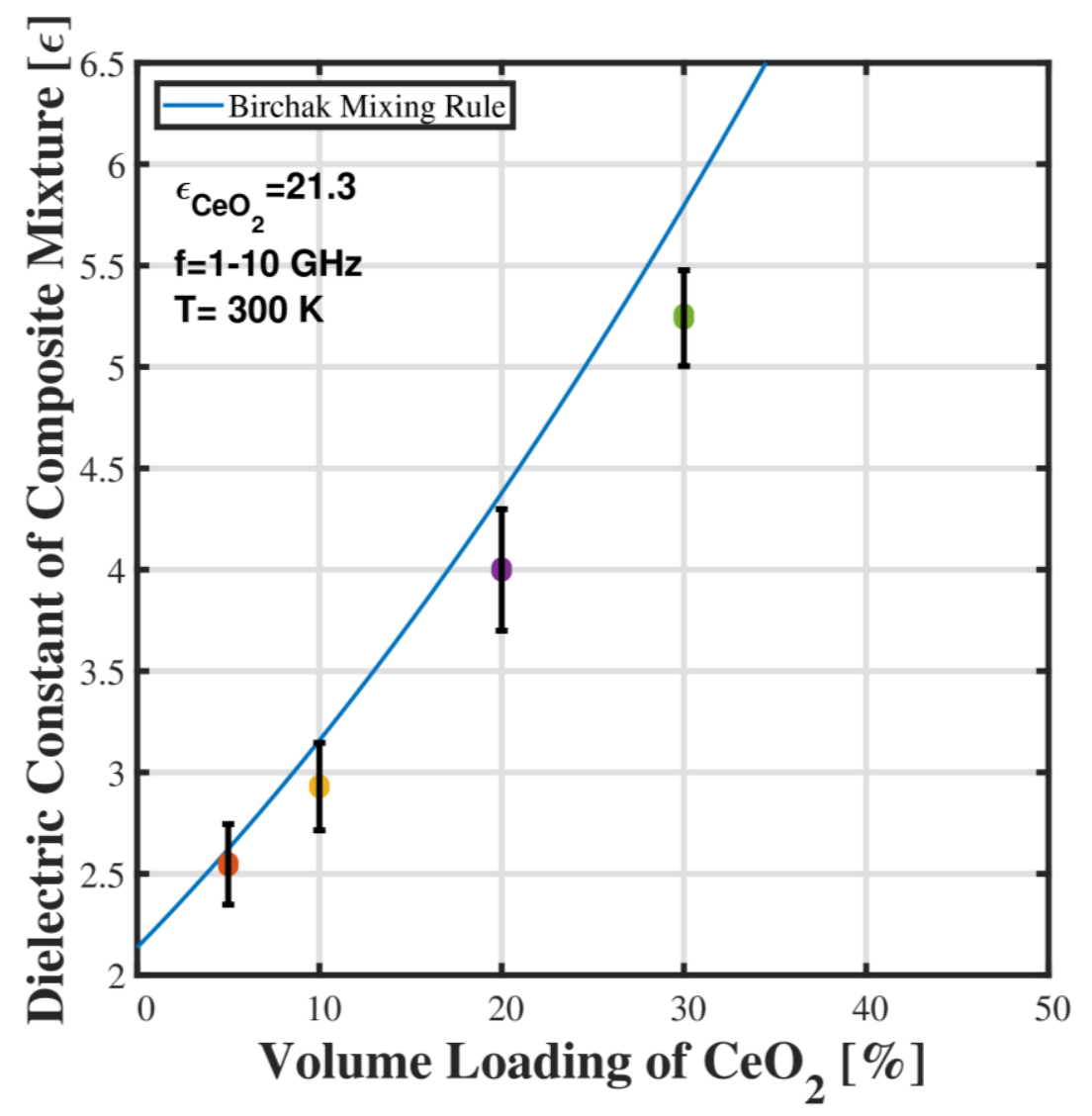

Figure 4.26: Theoretical Birchak mixture equation for $\mathrm{CeO}_{2}$ mapped against experimental scatter plot data for four different volume loadings 


\subsubsection{Theoretical Poon-Shin Mixing Equation for $\mathrm{CeO}_{2}$}

An evaluation of the performance of the Poon-Shin Mixing equation was completed and an analyzed. Like the other equations used for predictive analysis of this dielectric inclusion ratio excluding the Looyenga mixing rule, the Poon-Shin mixing equation is not extremely accurate. From Table 4.27 it can be seen that the mixture equation's accuracy is never within 5 percent error, with an average percent error of $12.04 \%$. This represents an increase for the error of the PoonShin Mixture equation by $6.54 \%$. An analysis of Figure 4.27 shows that this is another equation that does a decent job of mapping the growth of the dielectric constant of the compound as a function of volume loading but constantly under predicts the outputs.

Table 4.27: Absolute Percent Error for $\mathrm{CeO}_{2}$ composites using the Poon-Shin Mixing equation.

\begin{tabular}{|l|l|l|l|l|}
\hline $\mathrm{CeO}_{2}$ & $\mathbf{5 \%}$ VL & $\mathbf{1 0 \%}$ VL & $\mathbf{2 0 \%}$ VL & $\mathbf{3 0 \%}$ VL \\
\hline Percent Error & $6.32 \%$ & $8.68 \%$ & $15.86 \%$ & $17.29 \%$ \\
\hline
\end{tabular}

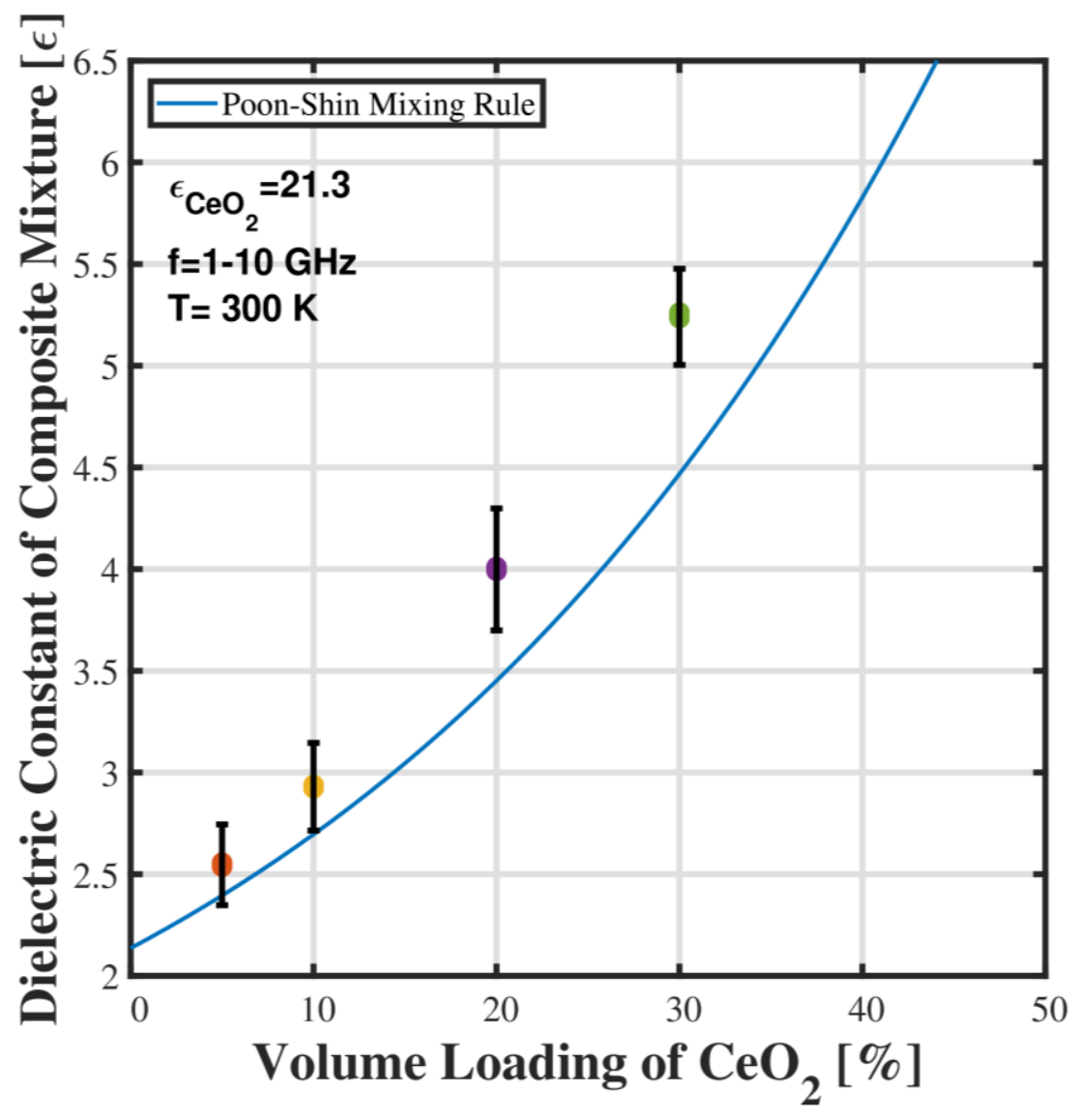

Figure 4.27: Theoretical Poon-Shin mixture equation for $\mathrm{CeO}_{2}$ mapped against experimental scatter plot data for four different volume loadings. 


\subsubsection{Theoretical Effective Medium Theory Mixing Equation for $\mathrm{CeO}_{2}$}

The EMT mixing equation is evaluated for its ability to accurately predict the dielectric constant as a function of volume loading. Unlike the other equations the EMT's shape factor variable allows it to take unique mathematical rates of change, with slower rates of change being expressed at higher volume loadings. The graph in Figure 4.28, reveals that the EMT mixing equation does not follow the trends of the experimental values at all. This is a significant change from the previous EMT section. A comparison of Table 4.28 shows that the percent error grows exponentially as volume loading increases, with an average percent error of $18.56 \%$. Compared to the usage of the EMT mixture equation in section 4.28 the average error percentage increased by $17.4 \%$ at this dielectric inclusion ratio.

Table 4.28: Absolute Percent Error for $\mathrm{CeO}_{2}$ composites using the EMT Mixing equation.

\begin{tabular}{|l|l|l|l|l|}
\hline $\mathrm{CeO}_{2}$ & 5\% VL & $\mathbf{1 0 \%}$ VL & $\mathbf{2 0 \%}$ VL & 30\% VL \\
\hline Percent Error & $8.65 \%$ & $6.18 \%$ & $15.40 \%$ & $44.02 \%$ \\
\hline
\end{tabular}

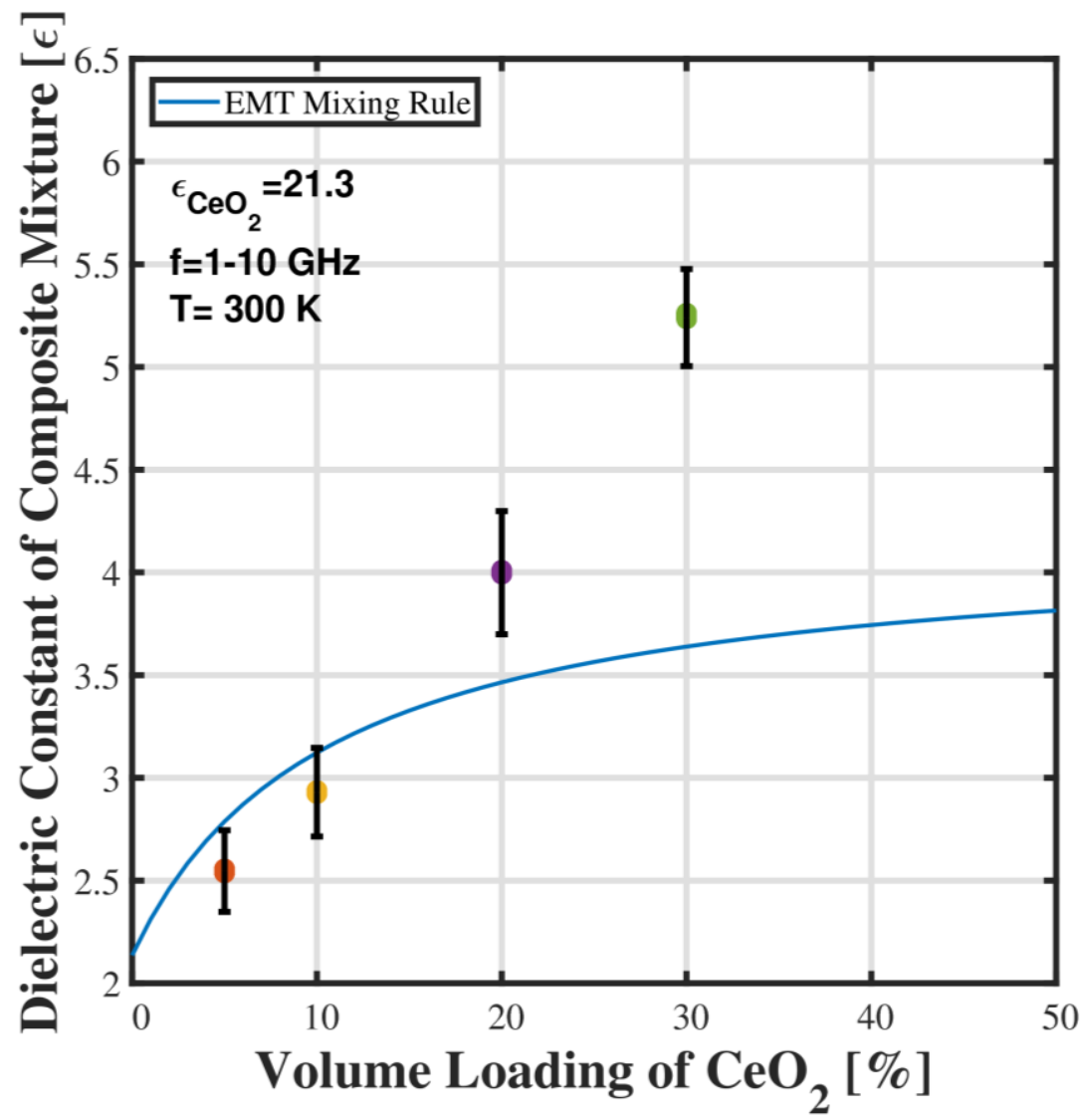

Figure 4.28: Theoretical EMT mixture equation for $\mathrm{CeO}_{2}$ mapped against experimental scatter plot data for four different volume loadings. 


\subsubsection{Theoretical Maxwell-Garnet Mixing Equation for $\mathrm{CeO}_{2}$}

The Maxwell-Garnet Mixing equation is assessed for its capability to map the increasing dielectric constant of the $\mathrm{CeO}_{2}$ loaded composite. It can be inferred from the graphical information in Figure 4.29 that the Maxwell-Garnet Mixture equation is not able to predict the dielectric constant of the mixture. It does follow the basic trends of the scatterplot but like many of the other equation in this section, it under predicts the outputs. A comparison with Table 4.29 shows quantitively that at each of the volume loading the equation is has a greater than 5\% accuracy, with an average percent error of $17.68 \%$. A decrease in prediction accuracy from section 4.2.9's of $13.31 \%$.

Table 4.29: Absolute Percent Error for $\mathrm{CeO}_{2}$ composites using the Maxwell-Garnet Mixing equation.

\begin{tabular}{|l|l|l|l|l|}
\hline $\mathrm{CeO}_{2}$ & $\mathbf{5 \%}$ VL & $\mathbf{1 0 \%}$ VL & $\mathbf{2 0 \%}$ VL & 30\% VL \\
\hline Percent Error & $6.74 \%$ & $10.35 \%$ & $22.4 \%$ & $31.19 \%$ \\
\hline
\end{tabular}

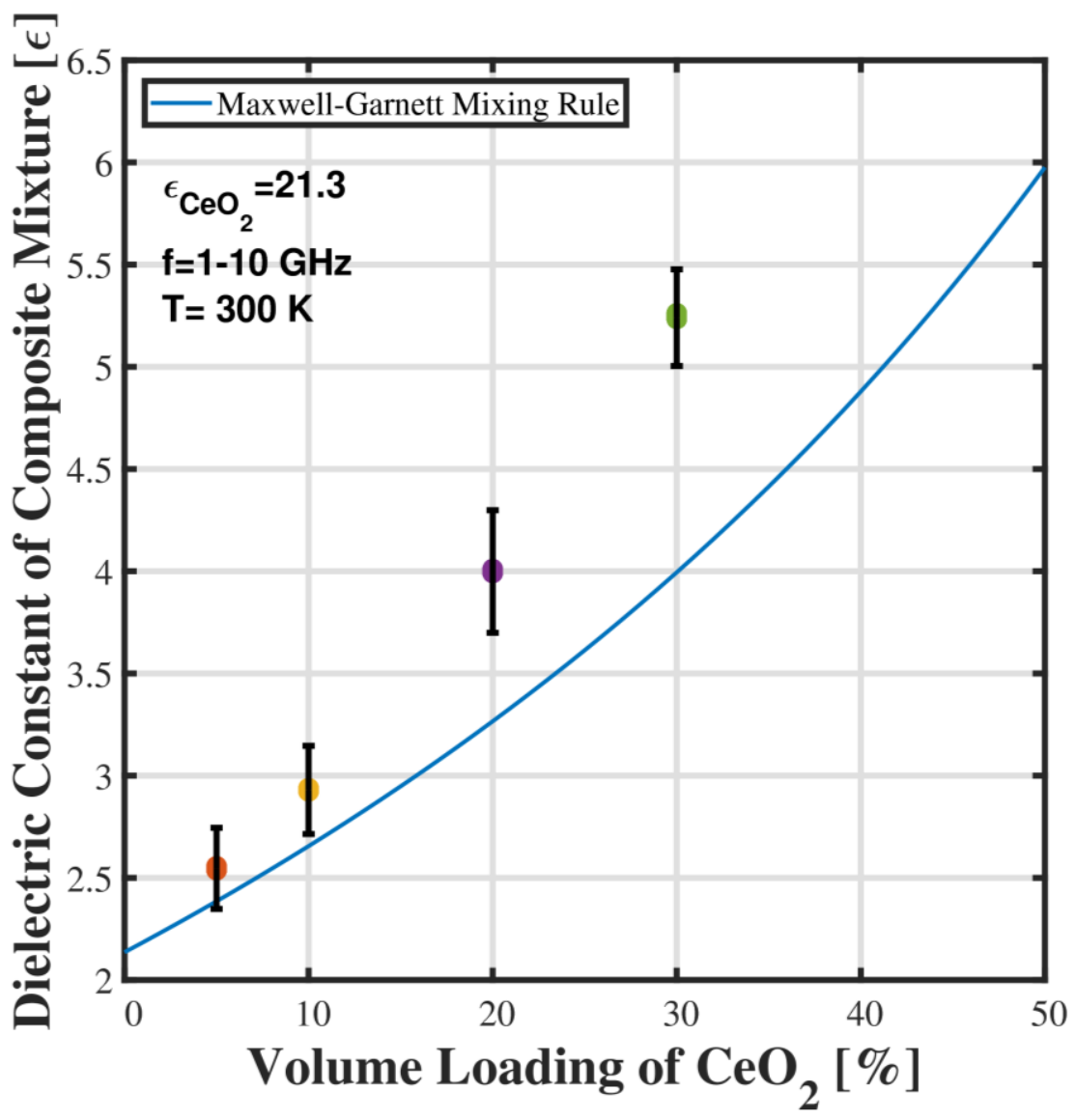

Figure 4.29: Theoretical Maxwell-Garnet mixture equation for $\mathrm{CeO}_{2}$ mapped against experimental scatter plot data for four different volume loadings. 


\subsubsection{Theoretical Jayasundere-Smith Mixing Equation for $\mathrm{CeO}_{2}$}

The final equation analyzed in this section was the Jayasundere-Smith mixing equation. Table 4.30 shows that the accuracy of the Looyenga mixing equation remains steadily constant as volume loading increases. With an overall error of $5.54 \%$ across all volume loadings, an increase of $2.16 \%$ from the previous powder. These results can be clearly clarified by a comparison with Figure 4.30, where the mixture equation passes through the t-distribution for all the volume loadings of the compounds However, the Jayasundere-Smith equation continuously under predicts the values of the compound like many of the other equations from this section.

Table 4.30: Absolute Percent Error for $\mathrm{CeO}_{2}$ composites using the Jayasundere-Smith Mixing equation.

\begin{tabular}{|l|l|l|l|l|}
\hline $\mathrm{CeO}_{2}$ & 5\% VL & $\mathbf{1 0 \%}$ VL & $\mathbf{2 0 \%}$ VL & $\mathbf{3 0 \%}$ VL \\
\hline Percent Error & $5.52 \%$ & $5.85 \%$ & $7.16 \%$ & $3.63 \%$ \\
\hline
\end{tabular}

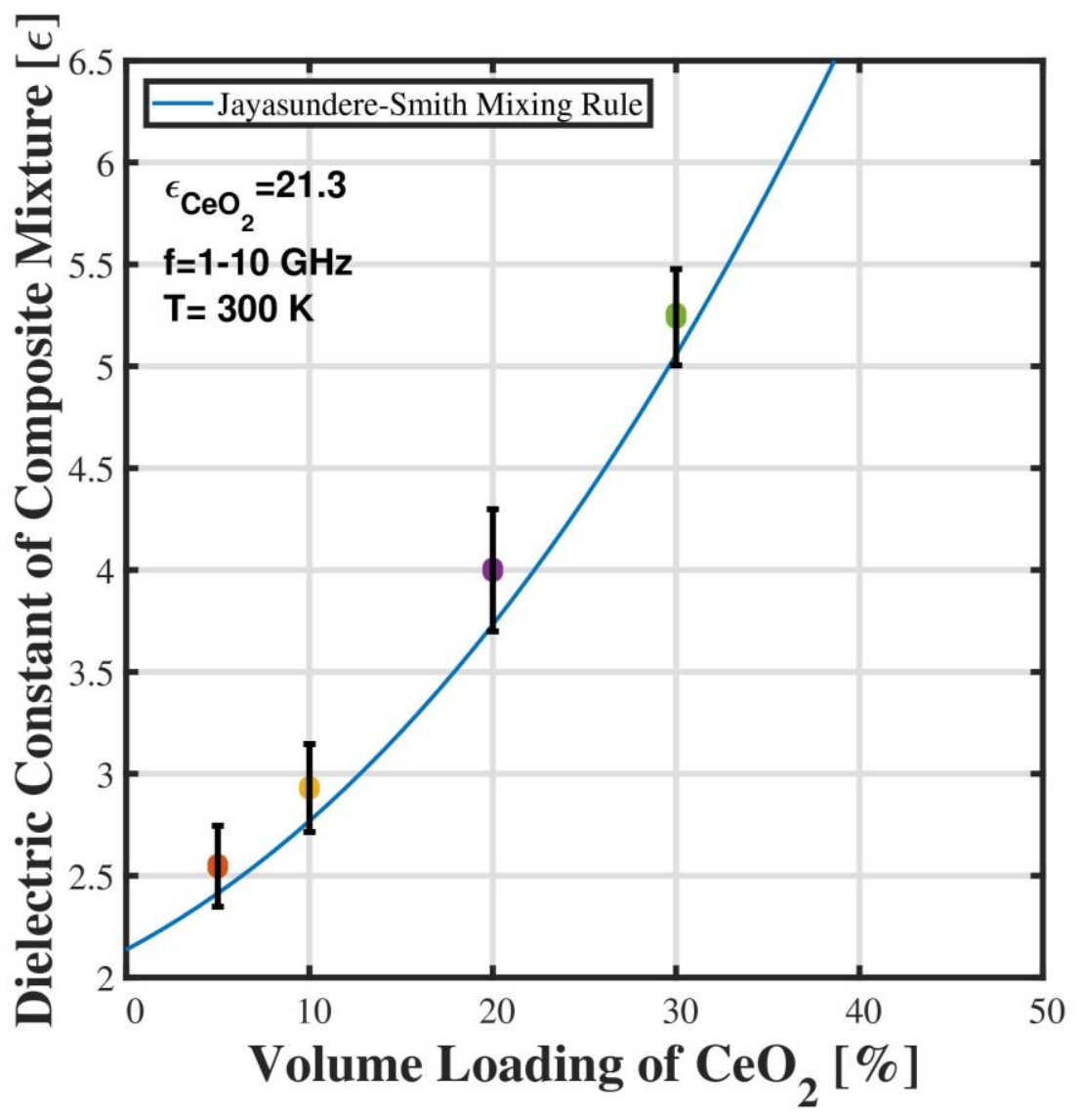

Figure 4.30: Theoretical Jayasundere-Smith mixture equation for $\mathrm{CeO}_{2}$ mapped against experimental scatter plot data for four different volume loadings. 


\subsection{Titanium Dioxide Composite Testing Results}

The last constitutive powder the study looked at was $\mathrm{TiO}_{2}$ for the purpose of ascertaining the accuracy of the mixture equations for this work. The higher dielectric constant of $\mathrm{TiO}_{2}\left(\varepsilon_{p}=\right.$ 70) results in a dielectric inclusion ratio of $\varepsilon_{p} / \varepsilon_{m}=30$. The theoretical outcomes of the mixture equations as a function of volume loading are graphed against the experimental scatter plot data in Figures 4.31-4.40 for $\mathrm{CeO}_{2}$. The absolute percent error between the different measured $\mathrm{CeO}_{2}$ composite and the theoretical output of each mixture equation are represented in Tables 4.31-4.40.

\subsubsection{Theoretical Parallel Mixing Equation Results for $\mathrm{TiO}_{2}$}

The study once again takes a look at the Parallel Mixing equation to ascertain its accuracy at this new dielectric inclusion ratio. The Parallel Mixing equation was found to have an average percent error across all volume loadings of $56.78 \%$. This is an increase of $28.79 \%$ error from the same equation using $\mathrm{CeO}_{2}$ as the filler material, a direct consequence of the increased dielectric inclusion ratio. Figure 4.31 and Table 4.31 shows that the parallel mixing rule does a terrible job of predicting the behavior of the composites dielectric constant as volume loading increases. It can be seen from the Figure that the behavior of the measured composite's dielectric constant behaves exponentially whilst the parallel mixture equation only predicts a linear growth.

Table 4.31: Absolute Percent Error for $\mathrm{TiO}_{2}$ composites using the Parallel Mixing equation.

\begin{tabular}{|l|l|l|l|l|}
\hline $\mathrm{CeO}_{2}$ & $\mathbf{5 \%}$ VL & $\mathbf{1 0 \%}$ VL & $\mathbf{2 0 \%}$ VL & 30\% VL \\
\hline Percent Error & $51.09 \%$ & $61.06 \%$ & $60.38 \%$ & $54.58 \%$ \\
\hline
\end{tabular}




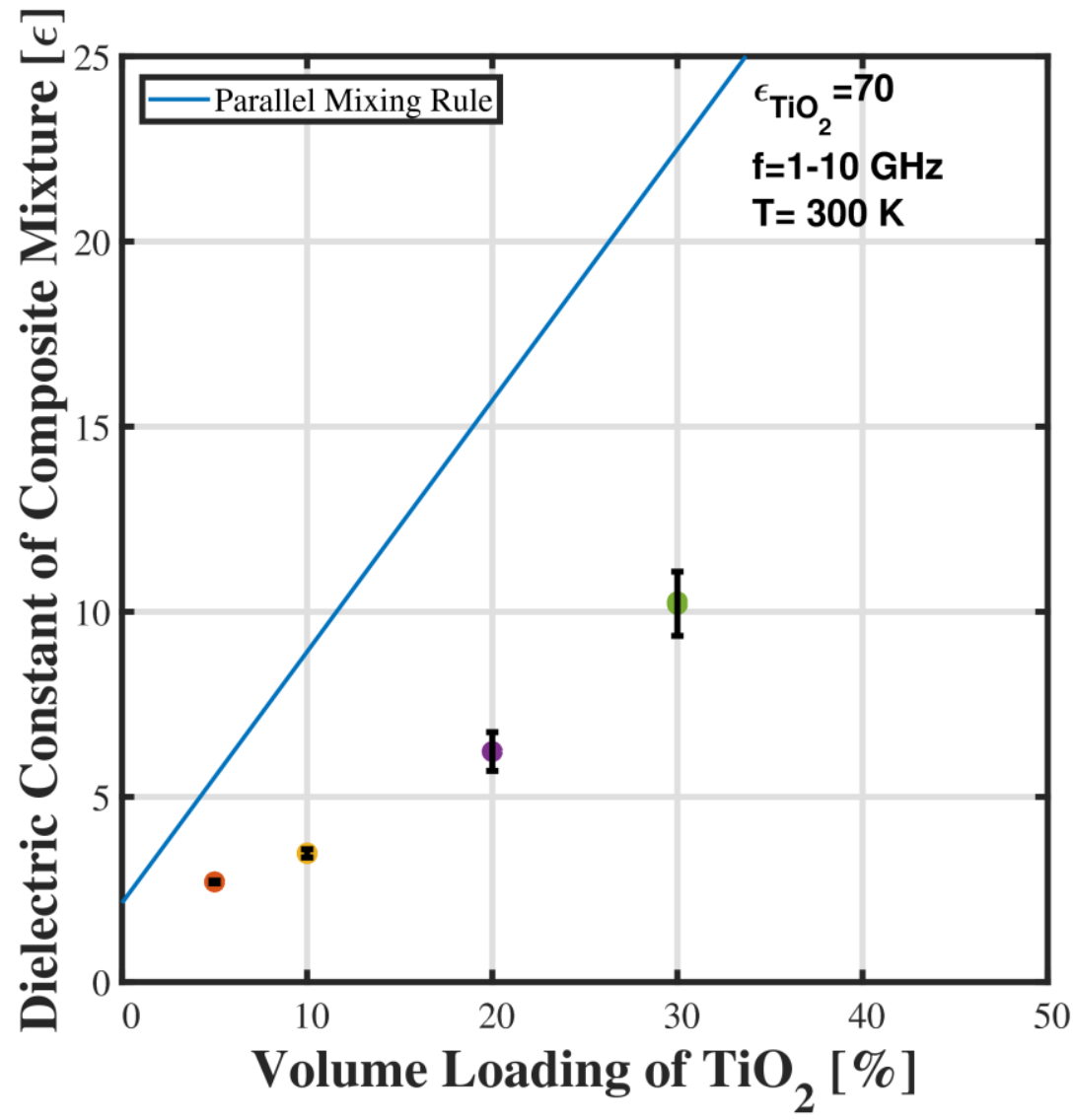

Figure 4.31: Theoretical Parallel mixture equation for $\mathrm{TiO}_{2}$ mapped against experimental scatter plot data for four different volume loadings. 


\subsubsection{Theoretical Series Mixing Equation Results for $\mathrm{TiO}_{2}$}

The next equation investigated is the series mixing equation for its validity in predicting the dielectric constant of the $\mathrm{TiO}_{2}$ composite. A visual scrutiny of the precision of the equation can be quickly surmised using Figure 4.32. This analysis can be quantified using Table 4.32, along with more details about the individual volume loadings accuracies. The average percent error of the Series mixing equation across all volume loadings was found to be $110.36 \%$. This is a $67.53 \%$ increase in error then what was seen for the $\mathrm{CeO}_{2}$ composites using the same equation. At none of the experimental measurement points does the series mixing equation pass within the t-distribution for this dielectric inclusion ratio.

Table 4.32: Absolute Percent Error for $\mathrm{TiO}_{2}$ composites using the Series Mixing equation.

\begin{tabular}{|l|l|l|l|l|}
\hline $\mathrm{CeO}_{2}$ & $\mathbf{5 \%}$ VL & $\mathbf{1 0 \%}$ VL & $\mathbf{2 0 \%}$ VL & 30\% VL \\
\hline Percent Error & $20.47 \%$ & $46.88 \%$ & $134.89 \%$ & $239.18 \%$ \\
\hline
\end{tabular}

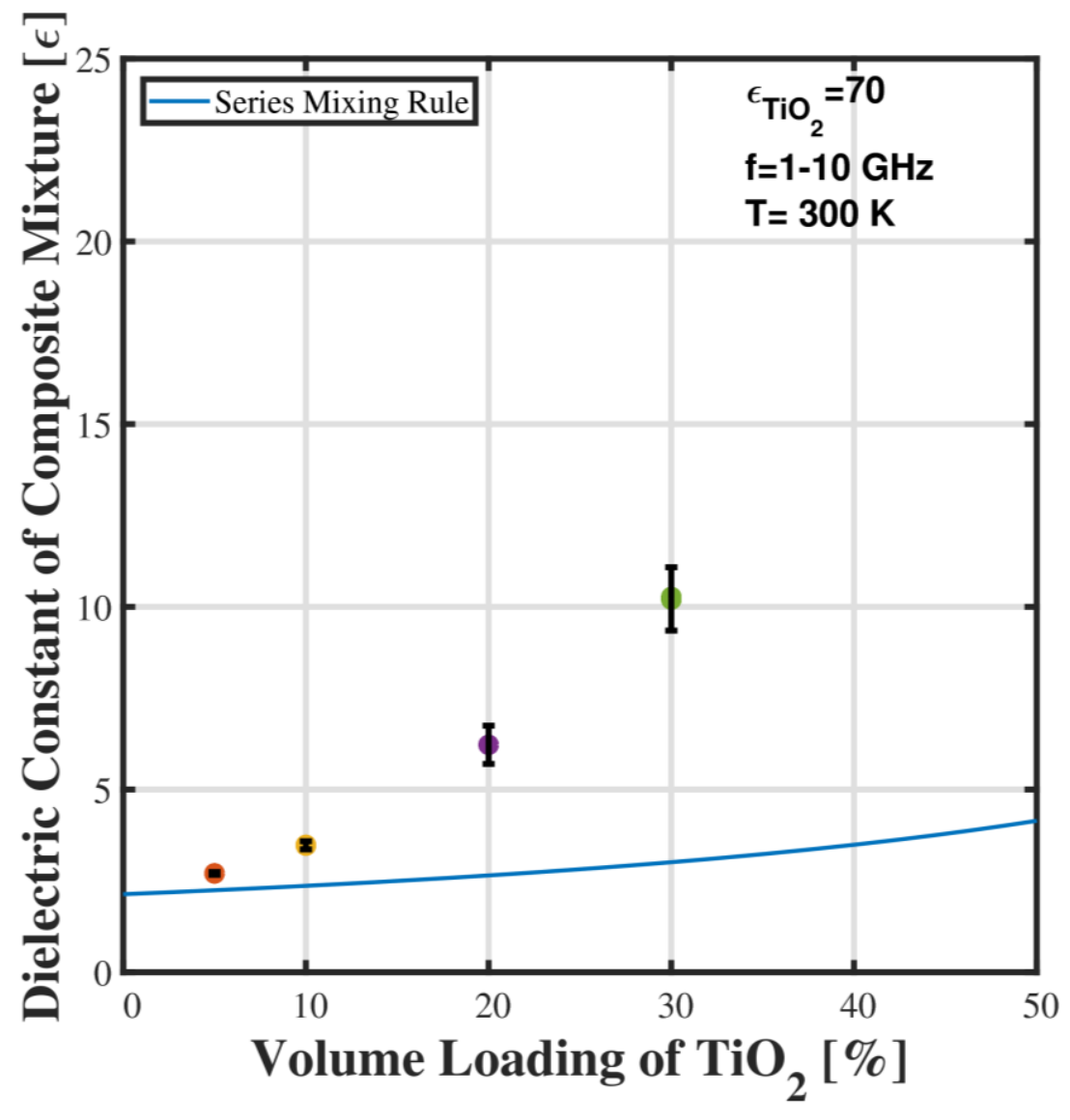

Figure 4.32: Theoretical Series mixture equation for $\mathrm{TiO}_{2}$ mapped against experimental scatter plot data for four different volume loadings. 


\subsubsection{Theoretical Logarithmic Mixing Equation Results for $\mathrm{TiO}_{2}$}

The Logarithmic Mixing equation is also evaluated for its ability to accurately predict the dielectric constant of a $\mathrm{TiO}_{2}$ composite as a function of volume loading. It can be summarized from the graphical information in Figure 4.33 that the logarithmic mixture equation is accurate at only a single volume loading represented on the graph. It comes close to following the general pattern of the increasing dielectric constant for this composite passing through the distribution at a single point. This can be seen from Table 4.33 at the volume loading of 10\%, an analysis of Table 4.23 shows that the average percent error is $40.39 \%$. This is an increase in accuracy from the usage of the same equation for $\mathrm{CeO}_{2}$ by a factor of $23.76 \%$. The increased dielectric inclusion ratio for $\mathrm{TiO}_{2}$ can be seen to directly affect the percent accuracy of each equation.

Table 4.33: Absolute Percent Error for $\mathrm{TiO}_{2}$ composites using the Logarithmic Mixing equation.

\begin{tabular}{|l|l|l|l|l|}
\hline $\mathrm{CeO}_{2}$ & 5\% VL & $\mathbf{1 0 \%}$ VL & $\mathbf{2 0 \%}$ VL & 30\% VL \\
\hline Percent Error & $17.88 \%$ & $1.00 \%$ & $49.12 \%$ & $93.56 \%$ \\
\hline
\end{tabular}

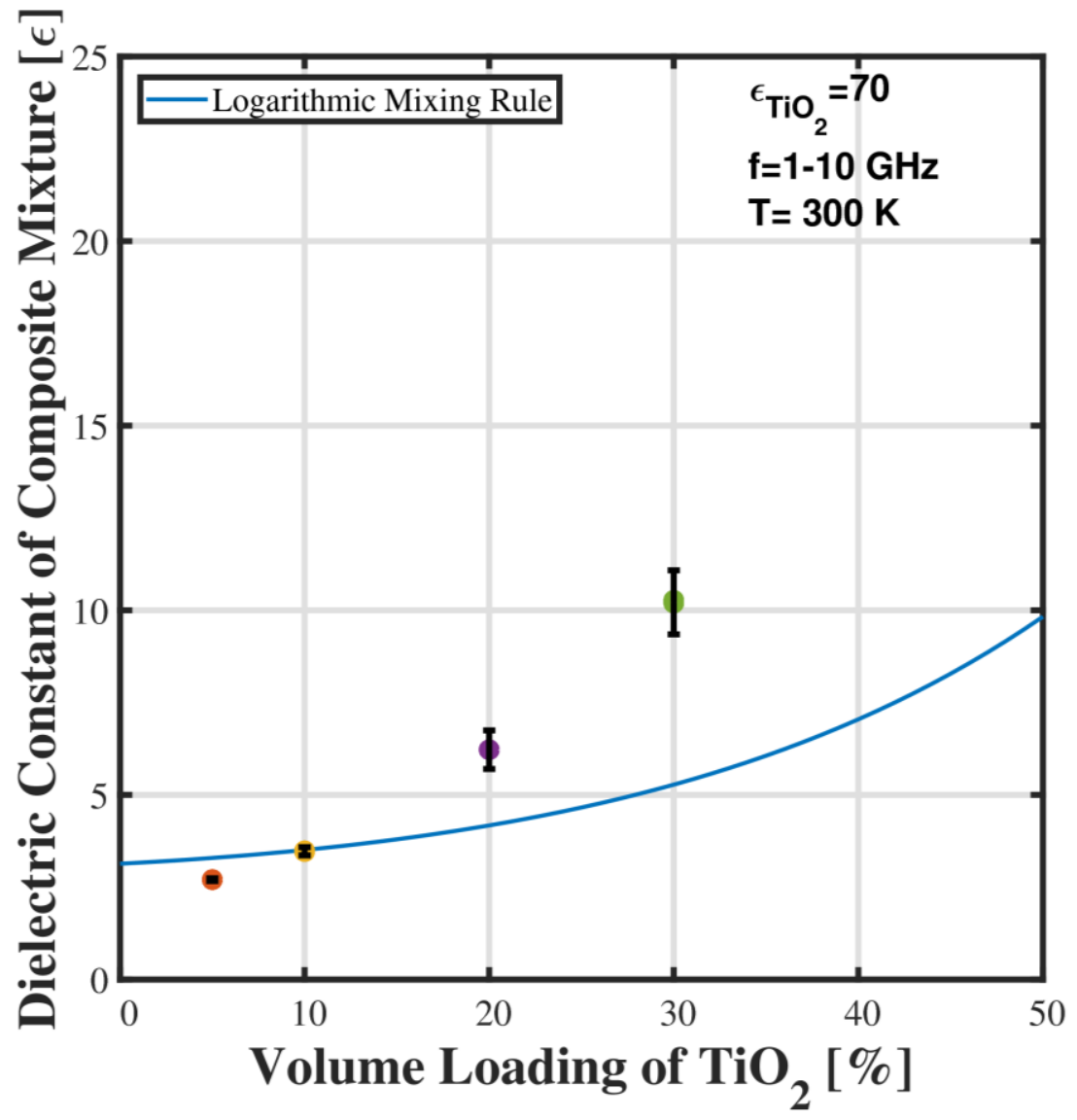

Figure 4.33: Theoretical Logarithmic mixture equation for $\mathrm{TiO}_{2}$ mapped against experimental scatter plot data for four different volume loadings. 


\subsubsection{Theoretical Lichtenecker Mixing Equation for $\mathrm{TiO}_{2}$}

The Lichtenecker mixing equation is again used in the evaluation of a two-phase homogenous composites ability to be mapped using a mixture. The Lichtenecker mixing equation struggles to map the behavior of the changes in dielectric constant as a function of volume loading. It has relatively accurate exponential growth for low volume loadings $(<20 \%)$ despite predicting a lower dielectric constant rate of change then what is seen experimentally. Like most of the equations looked at in the study at the higher volume loadings the accuracy of the equation begins to break down exponentially. The Lichtenecker equation has an average percent error of $33.49 \%$, this is an average accuracy prediction increase of $19.37 \%$ from the previous $\mathrm{CeO}_{2}$ powder using the Lichtenecker mixing equation. Figure 4.34 shows that the Lichtenecker mixing equation only passes through the t-distribution at the 5\% volume loading.

Table 4.34: Absolute Percent Error for $\mathrm{TiO}_{2}$ composites using the Lichtenecker Mixing equation.

\begin{tabular}{|l|l|l|l|l|}
\hline $\mathrm{CeO}_{2}$ & 5\% VL & $\mathbf{1 0 \%}$ VL & 20\% VL & 30\% VL \\
\hline Percent Error & $6.34 \%$ & $14.74 \%$ & $45.01 \%$ & $67.90 \%$ \\
\hline
\end{tabular}

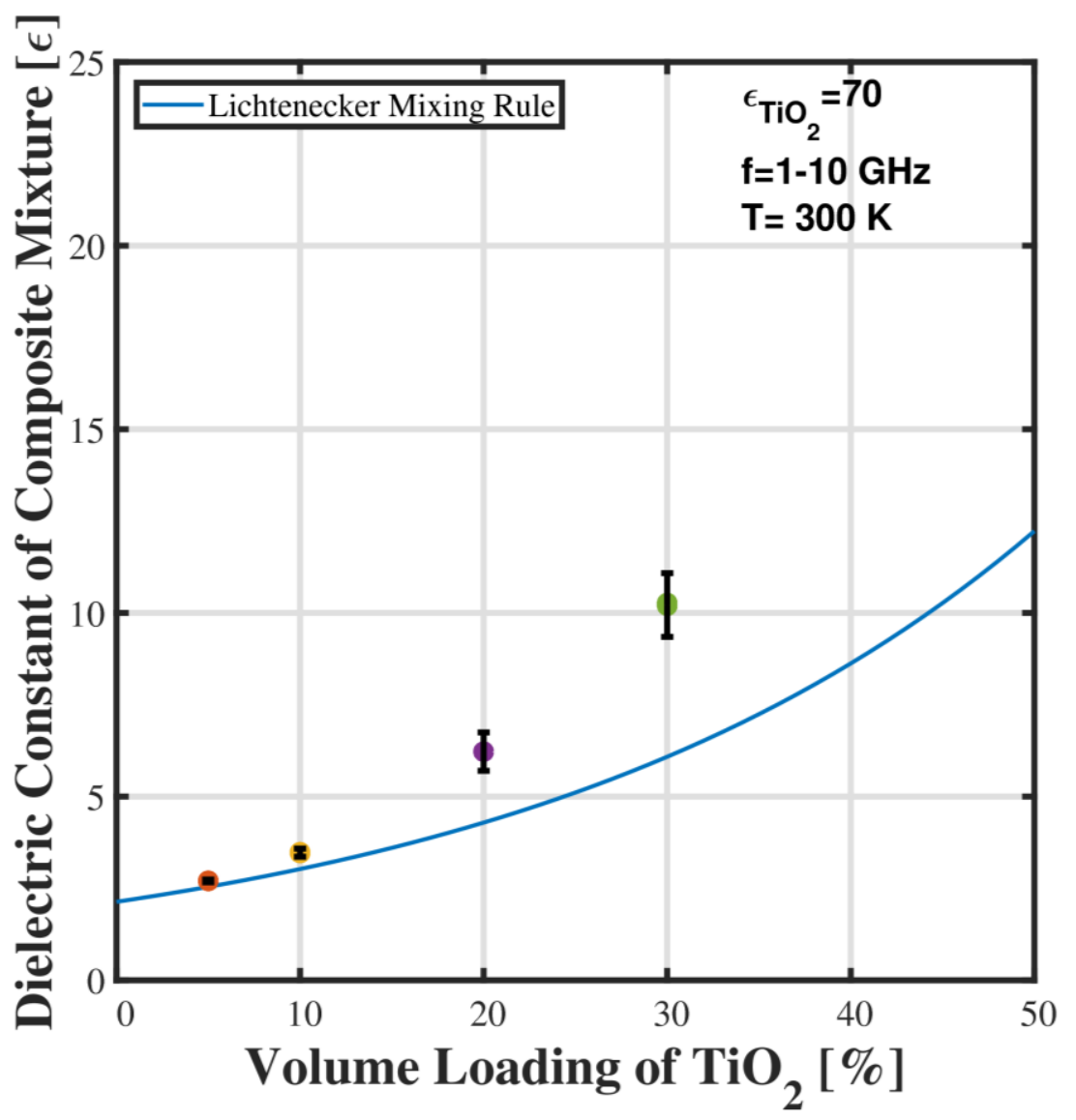

Figure 4.34: Theoretical Lichtenecker mixture equation for $\mathrm{TiO}_{2}$ mapped against experimental scatter plot data for four different volume loadings. 


\subsubsection{Theoretical Looyenga Mixing Equation for $\mathrm{TiO}_{2}$}

The ensuing equation examined is the Looyenga mixing equation for its accuracy in predicting the dielectric constant of the $\mathrm{TiO}_{2}$ composite. A visual examination of the accuracy of the equation can be quickly construed using Figure 4.35. This analysis can be quantified using Table 4.35. The average percent error of the Looyenga mixing equation across all volume loadings was found to be $6.21 \%$. This represents an extremely accurate equation for this application with the theoretical almost exactly predicting the compound's experimental measurements. The usage of the Looyenga mixing rule in predicting the output of the $\mathrm{TiO}_{2}$ compound represents an increase of 5.45 from the previous section but it is still much more accurate than any of the other equations looked at in this section.

Table 4.35: Absolute Percent Error for $\mathrm{TiO}_{2}$ composites using the Looyenga Mixing equation.

\begin{tabular}{|l|l|l|l|l|}
\hline $\mathrm{CeO}_{2}$ & $\mathbf{5 \%}$ VL & $\mathbf{1 0 \%}$ VL & $\mathbf{2 0 \%}$ VL & 30\% VL \\
\hline Percent Error & $7.43 \%$ & $10.43 \%$ & $2.42 \%$ & $4.55 \%$ \\
\hline
\end{tabular}

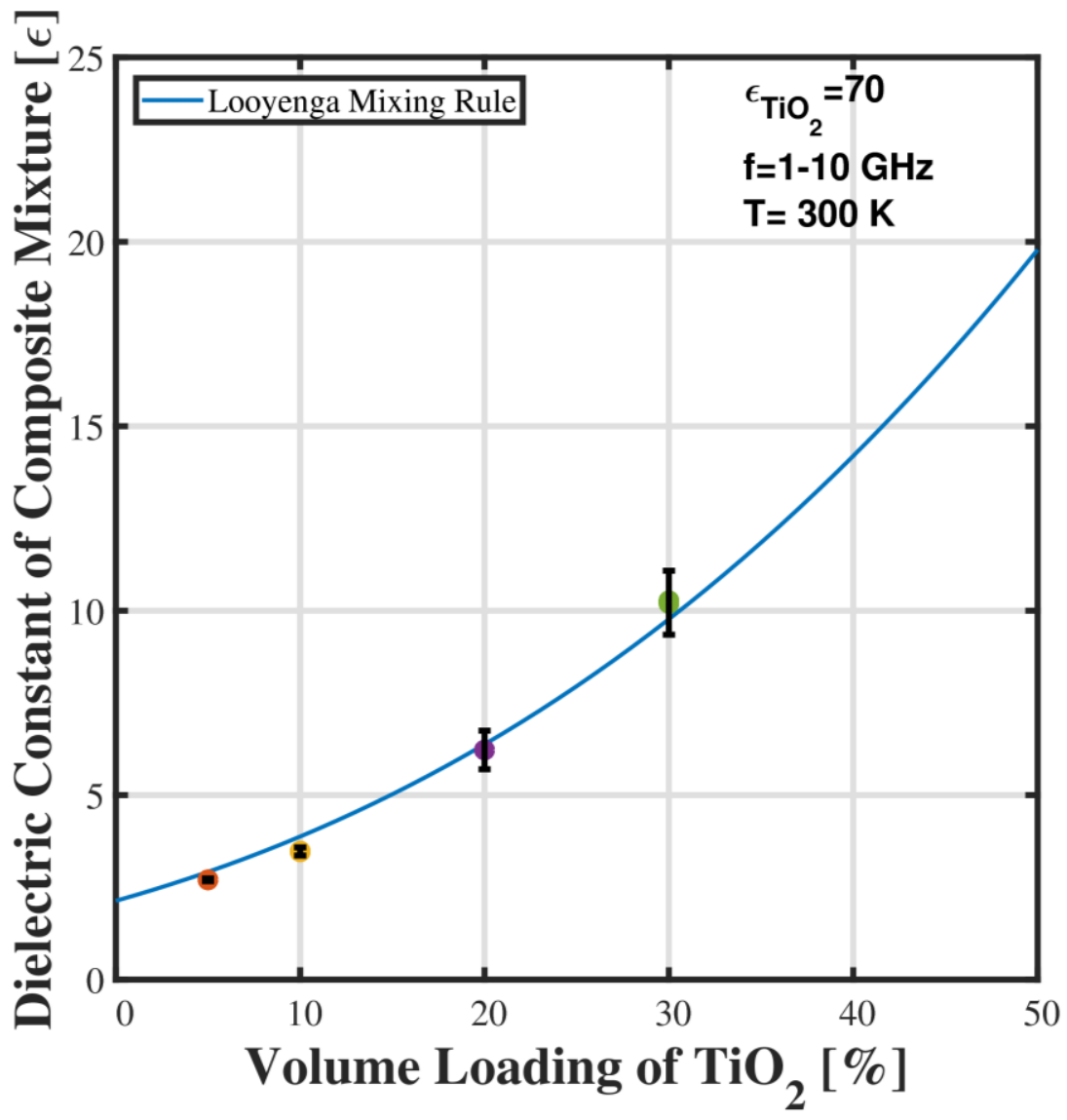

Figure 4.35: Theoretical Looyenga mixture equation for $\mathrm{TiO}_{2}$ mapped against experimental scatter plot data for four different volume loadings. 


\subsubsection{Theoretical Birchak Mixing Equation for $\mathrm{TiO}_{2}$}

Another evaluation of the $\mathrm{CeO}_{2}$ two-phase homogenous mixture equation's is accomplished using Birchak mixing equation the Birchak equation has an average percent error of $20.81 \%$. Once again this can be seen in Figure 4.36, where the Birchak mixing equation using varies outside of the t-distributions for all volume loadings. The Birchak equation does a good job of predicting the growth of the dielectric constant of the mixture but overpredicts the value at each of the measured points. At this higher dielectric inclusion ratio for $\mathrm{TiO}_{2}$ as compared to $\mathrm{CeO}_{2}$ the Birchak mixing equation is less accurate with a decreased average accuracy of $13.73 \%$.

Table 4.36: Absolute Percent Error for $\mathrm{TiO}_{2}$ composites using the Birchak Mixing equation.

\begin{tabular}{|l|l|l|l|l|}
\hline $\mathrm{CeO}_{2}$ & $\mathbf{5 \%}$ VL & $\mathbf{1 0 \%}$ VL & $\mathbf{2 0 \%}$ VL & 30\% VL \\
\hline Percent Error & $17.16 \%$ & $24.98 \%$ & $22.97 \%$ & $18.15 \%$ \\
\hline
\end{tabular}

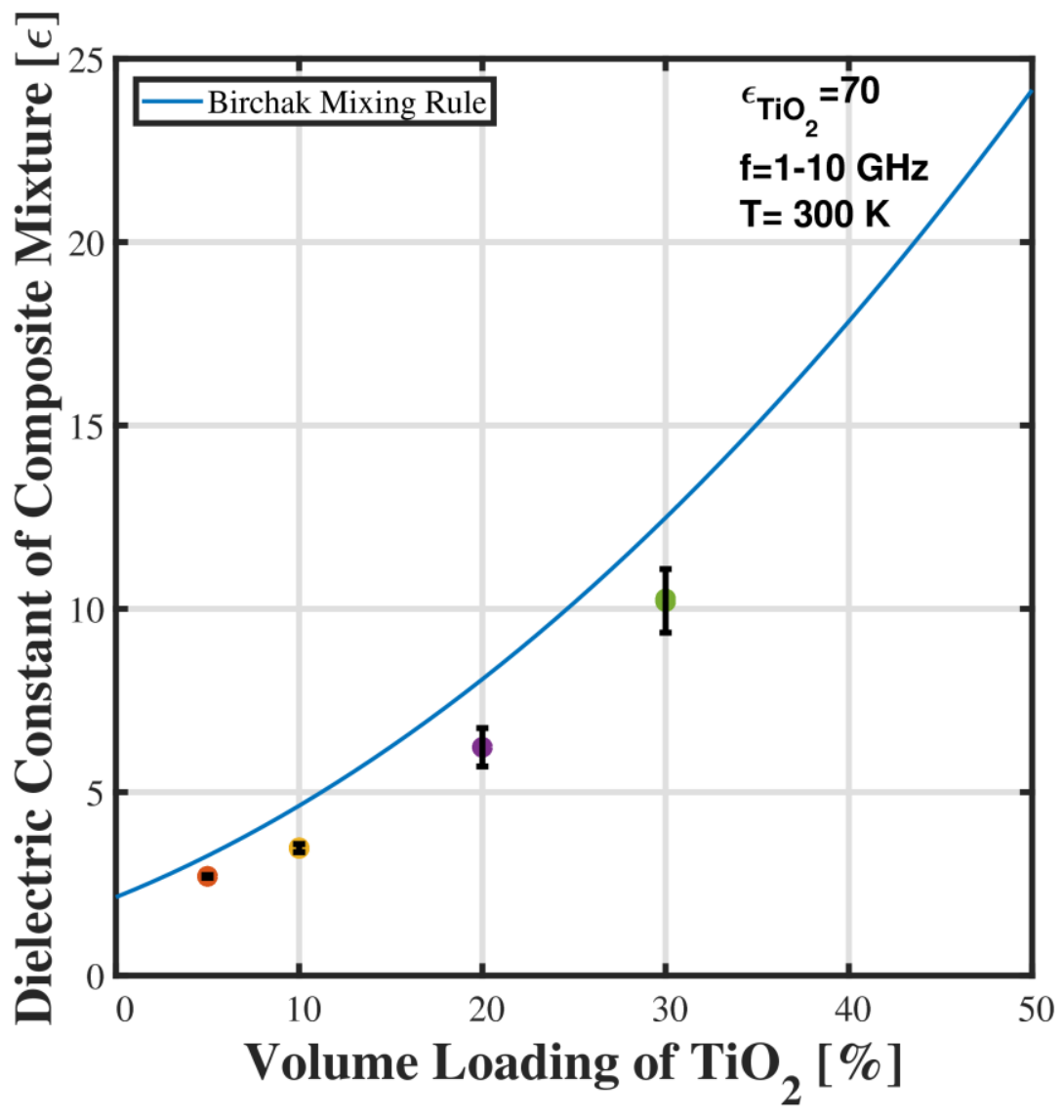

Figure 4.36: Theoretical Birchak mixture equation for $\mathrm{TiO}_{2}$ mapped against experimental scatter plot data for four different volume loadings. 


\subsubsection{Theoretical Poon-Shin Mixing Equation for $\mathrm{TiO}_{2}$}

An evaluation of the performance of the Poon-Shin Mixing equation was completed and validated. Like the other equations used for predictive analysis of this dielectric inclusion ratio excluding the Looyenga mixing rule, the Poon-Shin mixing equation is not extremely accurate. From Table 4.37 it can be seen that the mixture equation's accuracy is never within 10 percent error, with an average percent error of $45.22 \%$. This represents an increase for the error of the Poon-Shin Mixture equation by $33.18 \%$ over section 4.3.7. An analysis of Figure 4.37 shows that this is another equation that does a decent job of mapping the growth of the dielectric constant at low volume loadings $(<10 \%)$ of the compound as a function of volume loading but constantly under predicts the outputs.

Table 4.37: Absolute Percent Error for $\mathrm{TiO}_{2}$ composites using the Poon-Shin Mixing equation.

\begin{tabular}{|l|l|l|l|l|}
\hline $\mathrm{CeO}_{2}$ & 5\% VL & $\mathbf{1 0 \%}$ VL & $\mathbf{2 0 \%}$ VL & 30\% VL \\
\hline Percent Error & $10.05 \%$ & $22.14 \%$ & $60.38 \%$ & $88.30 \%$ \\
\hline
\end{tabular}

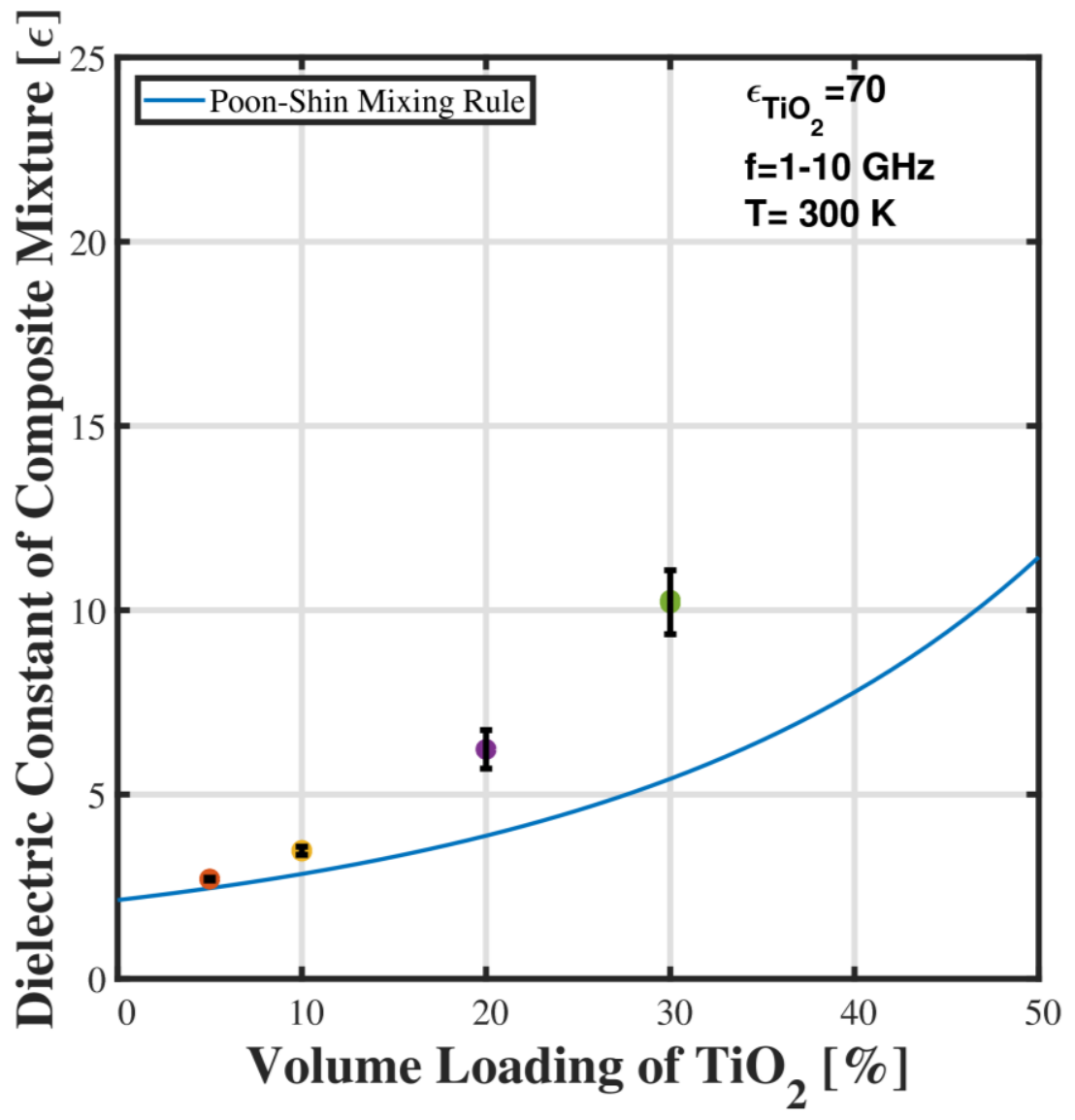

Figure 4.37: Theoretical Poon-Shin mixture equation for $\mathrm{TiO}_{2}$ mapped against experimental scatter plot data for four different volume loadings. 


\subsubsection{Theoretical Effective Medium Theory Mixing Equation for $\mathrm{TiO}_{2}$}

The EMT mixing equation is the next equation evaluated for it performance in predicting the outputs of volume loading. The graph in Figure 4.38, reveals that the EMT mixing equation does not follow the trends of the experimental values at all continuing the trend from the previous EMT section. A comparison of Table 4.38 confirms that the mixture equation is only applicable in the range of $10 \%$ volume loading compounds, with an average percent error of $60.62 \%$. Compared to the usage of the EMT mixture equation in section 4.38 the average error percentage increased by $42.06 \%$ at this dielectric inclusion ratio.

Table 4.38: Absolute Percent Error for $\mathrm{TiO}_{2}$ composites using the EMT Mixing equation.

\begin{tabular}{|l|l|l|l|l|}
\hline $\mathrm{CeO}_{2}$ & $\mathbf{5 \%}$ VL & $\mathbf{1 0 \%}$ VL & $\mathbf{2 0 \%}$ VL & 30\% VL \\
\hline Percent Error & $20.64 \%$ & $6.13 \%$ & $59.39 \%$ & $156.33 \%$ \\
\hline
\end{tabular}

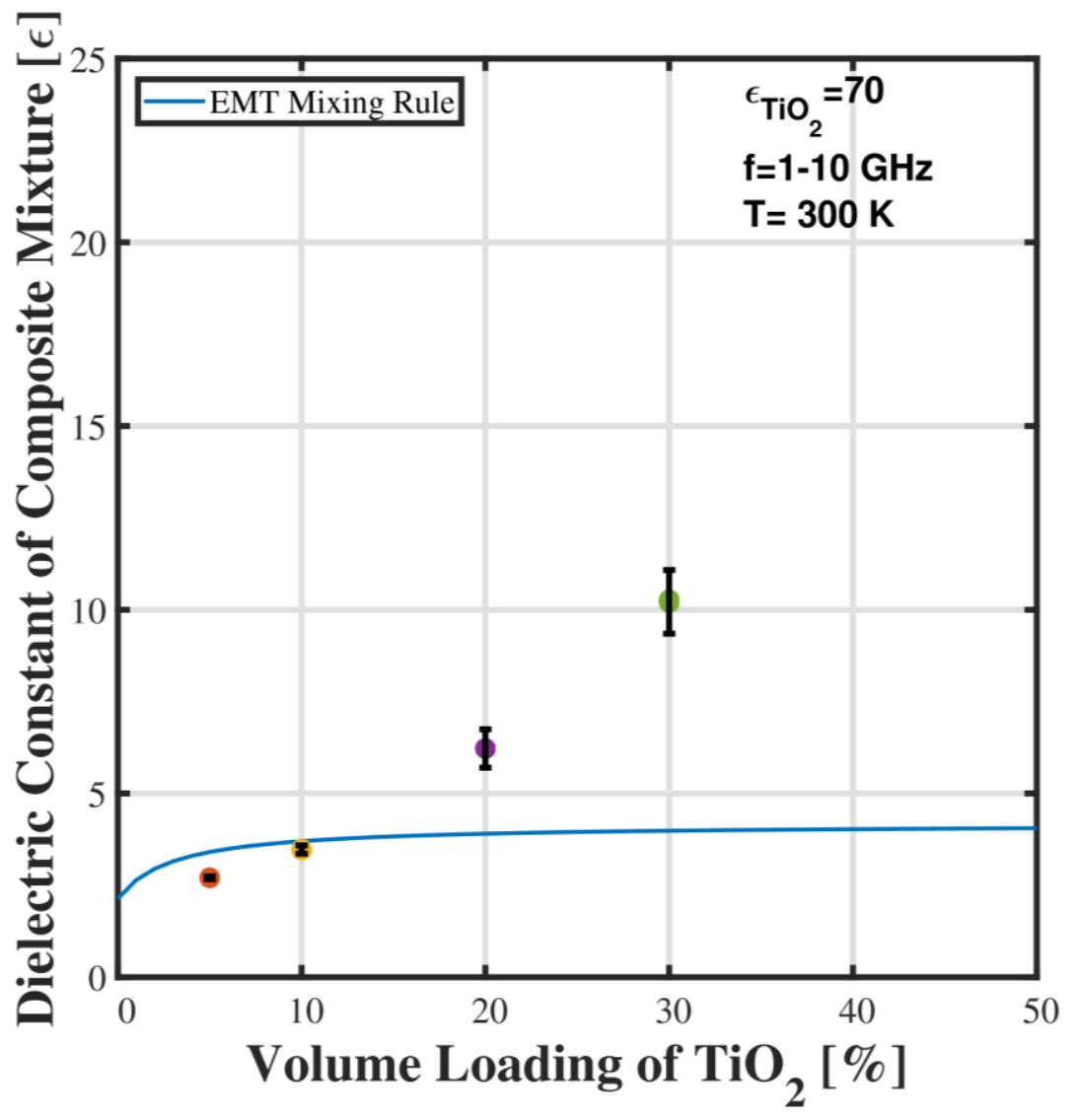

Figure 4.38: Theoretical EMT mixture equation for $\mathrm{TiO}_{2}$ mapped against experimental scatter plot data for four different volume loadings. 


\subsubsection{Theoretical Maxwell-Garnett Mixing Equation for $\mathrm{TiO}_{2}$}

The Maxwell-Garnet Mixing equation is assessed for its capability to map the increasing dielectric constant of the $\mathrm{TiO}_{2}$ loaded composite. It can be reasoned from the graphical information in Figure 4.39 that the Maxwell-Garnet Mixture equation is not able to predict the dielectric constant of the mixture. A comparison with Table 4.39 shows quantitively that at each of the volume loading the equation is has a greater than 5\% accuracy, with an average percent error of $58.58 \%$. A decrease in prediction accuracy from section 4.2 .9 's of $40.50 \%$. The equation under predicts the values of the dielectric with quickly diminishing accuracy as volume loading is increased.

Table 4.39: Absolute Percent Error for $\mathrm{TiO}_{2}$ composites using the Maxwell-Garnett Mixing equation.

\begin{tabular}{|l|l|l|l|l|}
\hline $\mathrm{CeO}_{2}$ & 5\% VL & $\mathbf{1 0 \%}$ VL & $\mathbf{2 0 \%}$ VL & 30\% VL \\
\hline Percent Error & $10.71 \%$ & $24.95 \%$ & $74.40 \%$ & $124.24 \%$ \\
\hline
\end{tabular}

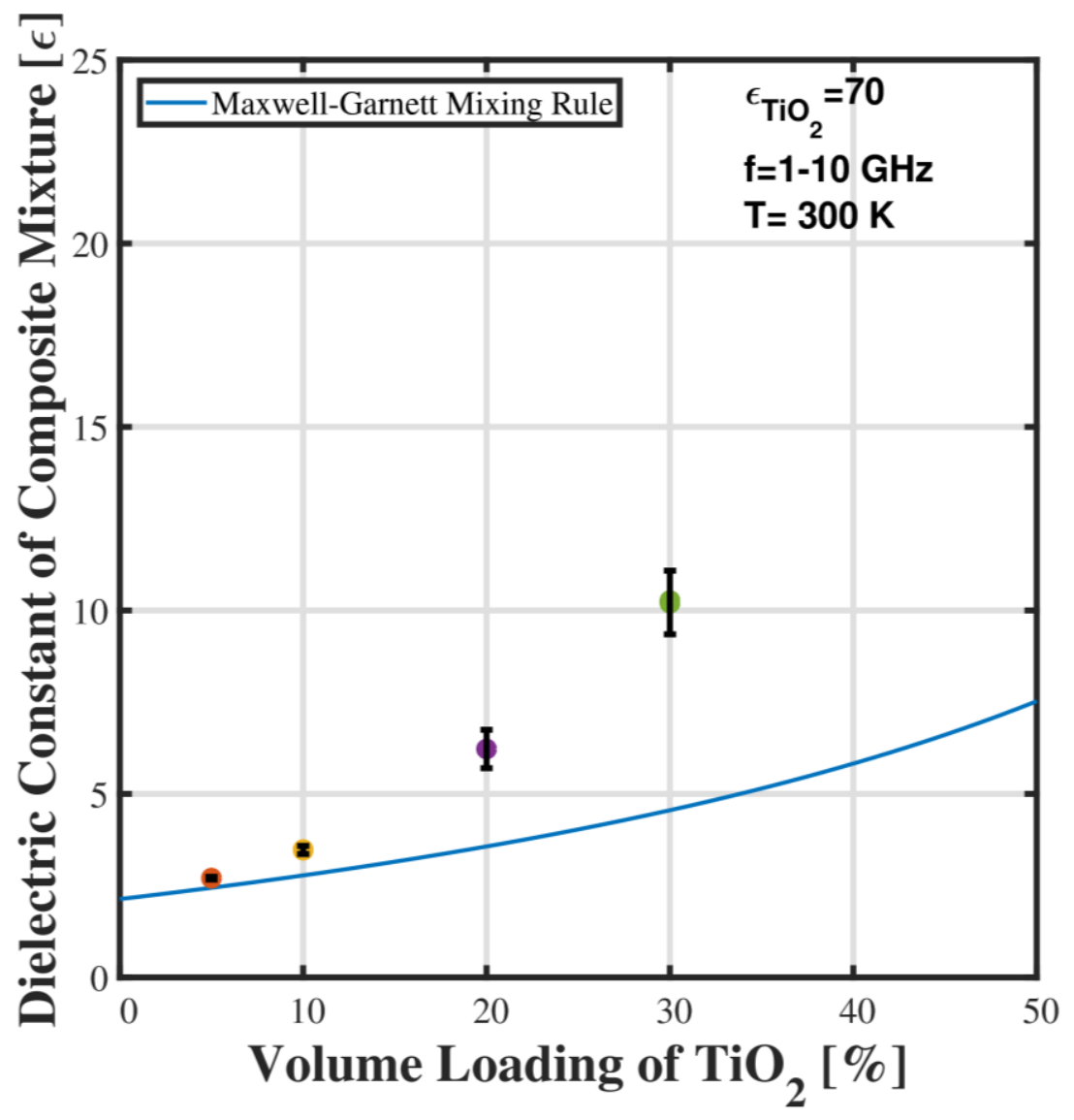

Figure 4.39: Theoretical Maxwell-Garnet mixture equation for $\mathrm{TiO}_{2}$ mapped against experimental scatter plot data for four different volume loadings. 


\subsubsection{Theoretical Jayasundere-Smith Mixing Equation for $\mathrm{TiO}_{2}$}

The final equation analyzed in this section was the Jayasundere-Smith mixing equation. Table 4.40 shows that the accuracy of the Looyenga mixing equation decreases steadily as volume loading increases, like practically all the equations evaluated in this section. The JayasundereSmith equation has an overall error of $32.33 \%$ across all volume loadings, an increase of $26.79 \%$ from the previous powder. These results can be clearly clarified by a comparison with Figure 4.40, where the mixture equation diverges from the measured values increasingly at high volume loadings. For this higher dielectric inclusion ratio, the Jayasundere-Smith equation constantly under predicts the value of the compound.

Table 4.40: Absolute Percent Error for $\mathrm{TiO}_{2}$ composites using the Jayasundere-Smith Mixing equation.

\begin{tabular}{|l|l|l|l|l|}
\hline $\mathrm{CeO}_{2}$ & 5\% VL & $\mathbf{1 0 \%}$ VL & 20\% VL & 30\% VL \\
\hline Percent Error & $8.95 \%$ & $17.57 \%$ & $43.78 \%$ & $59.13 \%$ \\
\hline
\end{tabular}

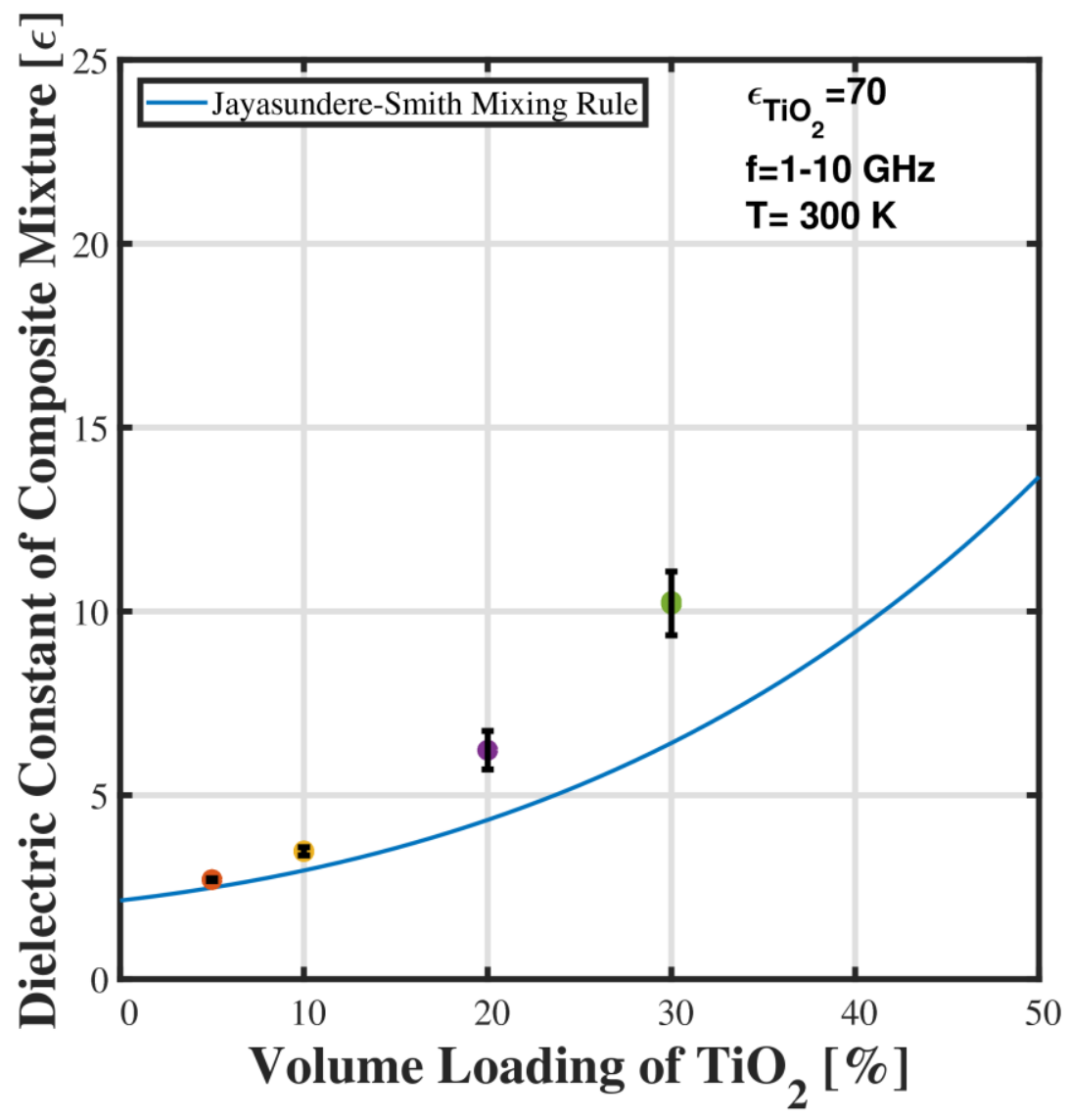

Figure 4.40: Theoretical Jayasundere-Smith mixture equation for $\mathrm{TiO}_{2}$ mapped against experimental scatter plot data for four different volume loadings. 


\section{CHAPTER 5: CONCLUSION}

\subsection{Summary of Research}

This study investigated a variety of powder materials with a wide range of dielectric properties in a coaxial transmission line. The objective was to gain an understanding of when to best utilize the multiple mixture equations in order to calculate a powder's dielectric constant $\left(\varepsilon_{p}\right)$ based on the experimental measurement of a composite mixture. In this study the mathematical validity of each mixing equation was tested independently for the four powdery materials using known dielectric constants from literature for the powders and a measured dielectric constant for the paraffin. It was determined that the selection of the most appropriate equation based on minimizing the percent error was dependent on the dielectric inclusion ratio's value, $\varepsilon_{p} / \varepsilon_{m}$. Table 5.1 and Table 5.2 provide a summary of the findings from this study. Table 1 provide the associated error and the appropriate mixing equation for the powdery materials studied. Table 2 provides guidance on selecting the correct mixing equation provided a given $\varepsilon_{p} / \varepsilon_{m}$.

It can be clearly seen from this study that as the dielectric inclusion ratio increases the associated error for each equation also increases. For $\varepsilon_{p} / \varepsilon_{m}=1.5\left(\mathrm{SiO}_{2}\right), \varepsilon_{p} / \varepsilon_{m}=4.3\left(\mathrm{Al}_{2} \mathrm{O}_{3}\right)$, $\varepsilon_{p} / \varepsilon_{m}=10\left(\mathrm{CeO}_{2}\right), \varepsilon_{p} / \varepsilon_{m}=30\left(\mathrm{CeO}_{2}\right)$, the averaged percent error for all the equations associated with that dielectric inclusion ratio were $3.59 \%, 8.17 \%, 16.32 \%$ and $46.48 \%$ respectively. This indicates that if using this method, it would be preferable to use a matrix material that resulted in the smallest $\varepsilon_{p} / \varepsilon_{m}$ possible. This would allow for the use of multiple equation to validate the results achieved using the ideal equation. This would also help for powdery materials of which very little is known as it would allow for a greater margin of error. 
Table 5.1: The best mixing equation for each powdery material studied and the associated averaged error over the volume fractions studied between the equation and the experimental values. The EMT mixing equation is recommended over the Maxwell-Garnett for simplicity despite a statistically insignificant improvement of error when using the latter equation.

\begin{tabular}{|c|c|c|}
\hline Powder & $\begin{array}{c}\text { Suggested Mixing } \\
\text { equation }\end{array}$ & Percent Error \\
\hline \multicolumn{2}{|c|}{} \\
\hline $\mathrm{SiO}_{2}$ & EMT & 0.94 \\
\hline $\mathrm{Al}_{2} \mathrm{O}_{3}$ & EMT & 1.16 \\
\hline $\mathrm{CeO}_{2}$ & Looyenga & 0.76 \\
\hline $\mathrm{TiO}_{2}$ & Looyenga & 6.21 \\
\hline
\end{tabular}

Table 5.2: Suggested mixing equation based on the ratio of powder's dielectric properties to matrix material's dielectric properties.

\begin{tabular}{|c|c|}
\hline $\begin{array}{c}\text { Ratio of Dielectric } \\
\text { Properties, } \varepsilon_{\mathrm{p}} / \varepsilon_{\mathrm{m}}\end{array}$ & $\begin{array}{c}\text { Suggested } \\
\text { Mixing } \\
\text { equation }\end{array}$ \\
\hline \multicolumn{2}{|c|}{} \\
\hline $1-10$ & EMT \\
\hline $10-100$ & Looyenga \\
\hline
\end{tabular}

This study also leads to the conclusion that lower volume loadings generally result in more accurate equations. For all the equations and powder materials in this study it was found that the average percent error was $8.80 \%, 11.29 \%, 21.09 \%$, and $33.28 \%$ for volume loadings of 5, 10, 20 and 30 percent respectively. Based on these results it is concluded that low volume loadings will allow for the most accurate calculations of the powdery materials dielectric based purely on the mathematics of the equations.

The method of using a coaxial transmission line to determine the room temperature relative dielectric constant of powder materials has several clear advantages over other transmission 
methods. The approach has clear advantages over surface probe methods that are susceptible to differential pressures and free space methods that required complicated calibration considerations, to permit powdery materials that are electrically conductivity to be measured using a coaxial method the powders were incorporating with non-conductive paraffin mixture. By keeping the volume loading within the state range and forming coaxial plugs in the manor described within the study, it is proposed that reliable dielectric measurements can be made with greater certainty than other methods.

It was found during testing that several material and preparation factors helped to improve the accuracy of the dielectric testing. Smaller particle sizes $(<65 \mu \mathrm{m})$ were preferred for composite preparations. Particle size of $30 \mu \mathrm{m}$ had the best distribution within the paraffin matrix as well as typically having the most accurate volume loadings. The smaller particle sizes helped to avoid any particle clustering that would have interfered with the dielectric constant of the mixture.

To avoid airgaps between the sample and inner/outer conductor special attention had to be paid to the effects of thermal expansion on the composites. As the paraffin composites cooled they experienced an expansion away from the center probe mold and into the outer sleeve of the casting mold. This resulted in the need to make the 3-D printed center conductor's diameter smaller than that of the actual conductor by $0.1 \mathrm{~nm}$. Having the samples outer diameter be slightly larger than that of the testing cell helped to ensure no airgaps existed between the sample and conducting walls. Since paraffin in soft, inserting the sample into the test cell shaved off excess paraffin resulted in a sample with the exact dimensions of the test cell.

\subsection{Future Directions}

This study looks to continue applying the methodology developed in the study of more complex materials. The same methodology can be applied to powders that are frequency dependent allowing for the same calculation to be done at each frequency of interest. This will allow for a more robust usage of this methodology as most material's dielectric constants are frequency dependent. The study will also begin to look at magnetic materials using this same method of paraffin-powder composites. Special work will have to be done to ensure no conglomeration of particles during the curing process, but no significant changes will need to be made to accommodate magnetic powders. Another direction of research will be high temperature dielectric testing of powders, as most materials dielectric constants are temperature dependent as well as frequency dependent. 


\section{References}

(1) Sihvola, A. Electromagnetic Mixing Formulas and Applications; 1999.

(2) Musho, T. D.; Wildfire, C.; Houlihan, N. M.; Sabolsky, E. M.; Shekhawat, D. Study of $\mathrm{Cu} 2 \mathrm{O}$ Particle Morphology on Microwave Field Enhancement. Materials Chemistry and Physics. 2018, pp 278-284. 2018

(3) Sphicopoulos, T.; Teodoridis, V.; Gardiol, F. Simple Nondestructive Method for the Measurement of Material Permittivity. J. Microw. Power 1985, 20 (3), 165-172.

(4) Stuchly, M. A.; Stuchly, S. S. Coaxial Line Reflection Methods for Measuring Dielectric Properties of Biological Substances at Radio and Microwave Frequencies-A Review. $J$. Microw. Power 1980, 43 (3), 165-172.

(5) Baker-jarvis, J.; Janezic, M. D.; Domich, P. D.; Geyer, R. G. Analysis of an Open-Ended Coaxial Probe with Lift-Off for Nondestructive Testing. 1994, 43 (5), 1-8.

(6) Wildfire, C.; Sabolsky, E. M.; Spencer, M. J.; Shekhawat, D. Solid-State Synthesis of YAG Powders through Microwave Coupling of Oxide/Carbon Particulate Mixtures. Ceramics International. 2017, pp 11455-11462. 2017

(7) Chung-Lun, L.; Jenq-Gong, D.; Bi-Shiou, C.; Wen-Hsi, L. Low-Temperature Sintering and Microwave Dielectric Properties of Anorthite-Based Glass-Ceramics. J. Eur. Ceram. Soc. 2002, 95 (8), 2230-2235.

(8) Alford, N. M. N.; Penn, S. J. Sintered Alumina with Low Dielectric Loss. J. Appl. Phys. 1996, 80 (10), 5895-5898.

(9) Higasi, K. Dielectric Investigation on Coals. II. Mixture of Coal Powder and Paraffin Wax. 1957, 30 (5), 546.

(10) Dionne, G. F.; Fitzgerald, J. F.; Aucoin, R. C. Dielectric Constants of Paraffin-Wax-TiO2 Mixtures. J. Appl. Phys. 1976, 47 (4), 1708-1709.

(11) Kelly, J. M.; Stenoien, J. O.; Isbell, D. E. Wave-Guide Measurements in the Microwave Region on Metal Powders Suspended in Paraffin Wax. J. Appl. Phys. 1953, 24 (3), 258262.

(13) GNU Free Documentation Licese, Version 1.2, Free Software Foundation. 2019

(12) Vainshtein, L. A. Electromagnetic Waves. Izd. Radio i Sviaz 1988, 00, 440.

(14) Purcell, E. M. Electricity and Magnetism, Second.; McGraw-Hill Book Company: New York, 1985.

(15) Horikoshi, S.; Schiffmann, R. F.; Fukushima, J.; Serpone, N. Microwave Chemical and Materials Processing: A Tutorial; 2017.

(16) Stuerga, D. Microwave-Material Interactions and Dielectric Properties, Key Ingredients for Mastery of Chemical Microwave Processes; 2008; Vol. 1. 
(17) Surati, M. A.; Jauhari, S.; Desai, K. R. A Brief Review: Microwave Assisted Organic Reaction. Arch. Appl. Sci. Res. 2012, 4 (1), 645-661.

(18) Fung, A. K.; Chen, K.-S.; Schutzer, and D. Microwave Scattering and Emission Models for Users, 1st ed.; Artech House: Norwood, 2010.

(19) Zangwill, A. Modern Electrodynamics; 2013.

(20) Katrib, J.; Folorunso, O.; Dodds, C.; Dimitrakis, G.; Kingman, S. W. Improving the Design of Industrial Microwave Processing Systems through Prediction of the Dielectric Properties of Complex Multi-Layered Materials. J. Mater. Sci. 2015.

(21) Sugawara, H.; Kashimura, K.; Hayashi, M.; Matsumuro, T.; Watanabe, T.; Mitani, T. Temperature Dependence and Shape Effect in High-Temperature Mi- Crowave Heating of Nickel Oxide Powders. Phys. B Phys. Condens. Matter 2015, 458, 35-39.

(22) Mishra, R. R.; Sharma, A. K. Microwave-Material Interaction Phenomena: Heating Mechanisms, Challenges and Opportunities in Material Processing. Composites Part A: Applied Science and Manufacturing. 2016, pp 78-97.

(23) Bartley, P. G.; Begley, S. B. A New Technique for the Determination of the Complex Permittivity and Permeability of Materials. 2010 IEEE Int. Instrum. Meas. Technol. Conf. I2MTC 2010 - Proc. 2010, 54-57.

(24) Arthur R. Von Hipple. Dielectric Materals and Applications; Arthur R. Von Hipple, Ed.; The MIT Press, 1952.

(25) Arthur R. Von Hipple, S. Ramo, J. R. W. Dielectrics and Waves. Fields and Waves in Mordern Radio 1955, 8 (2).

(26) Geyer, R. G.; Grosvenor, C. A.; Holloway, C. L.; Janezic, M. D.; Johk, R. T.; Kabos, P.; Baker-Jarvis, J. Measuring the Permittivity and Permeability of Lossy Materials :; 2005.

(27) Hashin, Z.; Shtrikman, S. A Variational Approach to the Theory of the Effective Magnetic Permeability of Multiphase Materials. J. Appl. Phys. 1962, 33 (10), 3125-3131.

(28) Ebara, H.; Inoue, T.; Hashimoto, O. Measurement Method of Complex Permittivity and Permeability for a Powdered Material Using a Waveguide in Microwave Band. Sci. Technol. Adv. Mater. 2006, 7 (1 SPEC. ISS.), 77-83.

(29) Blakney, T. L.; Weir, W. B. Automatic Measurement of Complex Dielectric Constant and Permeability at Microwave Frequencies. Proc. IEEE 1975, 63 (1), 203-205.

(30) Choy, T. C. Effective Medium Theory: Principles and Applications, 2nd ed.; Oxford University Press, 2015.

(31) Anderson, R. W. S-Parameter Techniques For Faster, More Accurate Network Design; 1967.

(32) Agilent Technologies. Advanced Design System 1.5 Circuit Simulatione; Palo Alto, 2000.

(33) Jilani, M. T.; Rehman, M. Z. ur; Khan, A. M.; Khan, M. T.; Ali, S. M. A Brief Review of Measuring Techniques for Characterization of Dielectric Materials. Int. J. Inf. Technol. 
Electr. Eng. 2012, 1 (1), 1-5.

(34) Venkatesh, M. S.; Raghavan, G. S. V. An Overview of Dielectric Properties Measuring Techniques. J. Can. Soc. Bioeng. 2005, 47 (40), 7.15-7.30.

(35) Yaw, K. C. Measurement of Dielectric Material Properties Application Note, Rohde \& Schwarz. Meas. Tech. 2006, 1-35.

(36) Technologies, K. N5231A PNA-L Microwave Network Analyzer, $13.5 \mathrm{GHz}$

(37) Bussey, H. E. Measurement of RF Properties of Materials A Survey. Proc. IEEE 1967, 55 (6).

(38) Busseyt, H. E. Measurement and Standardization of DieIectric Sam\&; 1962, No. 4, 4-7.

(39) Nicolson, A. M.; Ross, G. F. Measurement of the $\{$ Intrinsic\} \{Properties $\}$ of $\{$ Materials by $\{$ Time $\}-\{$ Domain $\}$ \{Techniques $\}$. IEEE Trans. Instrum. Meas. 1970, 19 (4), 377-382.

(40) Agilent Technologies. Models in the 85071E Materials Measurement Software.

(41) Baker-Jarvis, J.; Janezic, M. D.; Grosvenor Jr, J. H.; Geyer, R. G. Transmission/Reflection and Short-Circuit Line Methods for Measuring Permittivity and Permeability; OFFICE, U. S. G. P., Ed.; Washington D.C., 1992.

(42) Catalá-civera, J. M.; Member, S.; Canós, A. J.; Plaza-gonzález, P.; Gutiérrez, J. D.; Garcíabaños, B.; Peñaranda-foix, F. L.; Member, S. Dynamic Measurement of Dielectric Properties of Materials at High Temperature During Microwave Heating in a Dual Mode Cylindrical Cavity. 2015, 63 (9), 2905-2914.

(43) Thommes, M.; Shields, J. E.; Thomas, M. A. Characterization of Porous Solids and Powders: Surface Area, Pore Size and Density, 1st ed.; Shields, J. E., Thomas, M. A., Lowell, S., Eds.; Springer Science \& Buisness Media, 2004.

(44) Castellanos, A. The Relationship between Attractive Interparticle Forces and Bulk Behaviour in Dry and Uncharged Fine Powders; 2005; Vol. 54.

(45) Javanbakht, M.; Salahinejad, E.; Hadianfard, M. J. The Effect of Sintering Temperature on the Structure and Mechanical Properties of Medical-Grade Powder Metallurgy Stainless Steels. Powder Technol. 2016, 289, 37-43.

(46) Ahmad, S. I.; Koteshwar Rao, P.; Syed, I. A. Sintering Temperature Effect on Density, Structural and Morphological Properties of Mg- and Sr-Doped Ceria. J. Taibah Univ. Sci. 2016, 10 (3), 381-385.

(47) Sandi, D.; Supriyanto, A.; Jamaluddin, A.; Iriani, Y. The Effects of Sintering Temperature on Dielectric Constant of Barium Titanate (BaTiO3). IOP Conf. Ser. Mater. Sci. Eng. 2016, 107 (1), 1-7.

(48) Costa, A. M. L. M.; Baroni, D. B.; Lucas, C. S.; Bittencourt, M. S. Q. ULTRASONIC ANALYSIS OF SINTERED ALUMINA PELLETS. 2016, No. May.

(49) Vattur Sundaram, M. Processing Methods for Reaching Full Density Powder Metallurgical Materials. 2017, 39. 
(50) Donachie Jr., M. J.; Burr, M. F. Effects of Pressing on Metal Powders. J. Met. 1963, 14, 849-850.

(51) Essa, K.; Jamshidi, P.; Zou, J.; Attallah, M. M.; Hassin, H. Porosity Control in 316L Stainless Steel Using Cold and Hot Isostatic Pressing. Mater. Des. 201AD, 138 (15), 2129.

(52) Taylor, P.; Himran, S.; Suwono, A.; Mansoori, G. A. L. I. Characterization of Alkanes and Paraffin Waxes for Application as Phase Change Energy Storage Medium Characterization of Alkanes and Paraf' Fin Waxes for Application as Phase Change Energy Storage Medium. 1994, 16 (August 2014), 37-41.

(53) Gasia, J.; Miró, L.; de Gracia, A.; Barreneche, C.; Cabeza, L. F. Experimental Evaluation of a Paraffin as Phase Change Material for Thermal Energy Storage in Laboratory Equipment and in a Shell-and-Tube Heat Exchanger. Appl. Sci. 2016, 6 (5).

(54) Chen, W. S.; Hsieh, M. Y. Dielectric Constant Calculation Based on Mixture Equations of Binary Composites at Microwave Frequency. Ceram. Int. 2017.

(55) Sihvola, A. Mixing Rules with Complex Dielectric Coefficients. Subsurf. Sens. Technol. Appl. 2000, 1 (4), 393-515.

(56) Simpkin, R. Derivation of Lichtenecker's Logarithmic Mixture Formula from Maxwell's Equations. IEEE Trans. Microw. Theory Tech. 2010, 58 (3), 545-550.

(57) Poon, Y. M.; Shin, F. G. A Simple Explicit Formula for the Effective Dielectric Constant of Binary 0-3 Composites. J. Mater. Sci. 2004, 39 (4), 1277-1281.

(58) Mallet, P.; Guérin, C. A.; Sentenac, A. Maxwell-Garnett Mixing Rule in the Presence of Multiple Scattering: Derivation and Accuracy. Phys. Rev. B - Condens. Matter Mater. Phys. 2005, 72 (1), 1-9.

(59) Tuhkala, M.; Juuti, J.; Jantunen, H. Method to Characterize Dielectric Properties of Powdery Substances. J. Appl. Phys. 2013, 114 (1).

(60) Jayasundere, N.; Smith, B. V. Dielectric Constant for Binary Piezoelectric 0-3 Composites. J. Appl. Phys. 1993, 73 (5), 2462-2466.

(61) Sigma-Aldrich. SiO2, 2017

(62) Samsonov, G. V. The Oxide Handbook, 2nd ed.; New York, 1982.

(63) Inframat. A12O3, 2016

(64) Hayat, A.; Andreescu, D.; Bulbul, G.; Andreescu, S. Redox Reactivity of Cerium Oxide Nanoparticles against Dopamine. J. Colloid Interface Sci. 2014, 418, 240-245.

(65) Scientific, F. Titanium(IV) oxide, Aeroxide ${ }^{\mathrm{TM}}$ P25, ACROS Organics ${ }^{\mathrm{TM}}, 2017$

(66) Schindelin, J.; Arganda-Carreras, I.; Frise. Fiji: An Open-Source Platform for BiologicalImage Analysi. Nat. Methods 2012, 9 (7), 676-682.

(67) Mauritz, K. Dielectric Spectroscopy. PSRC.edu, 2012 
(68) Smiths Interconnect. Linear-circular Polarizers and Circular-to-rectangular Waveguide, 2017 